\title{
Trace- and Failure-Based Semantics for Responsiveness
}

\author{
Walter Vogler ${ }^{1}$ and Christian Stahl ${ }^{2}$ and Richard Müller ${ }^{2,3}$ \\ 1 Institut für Informatik, Universität Augsburg, Germany \\ vogler@informatik . uni-augsburg.de \\ 2 Department of Mathematics and Computer Science, \\ Technische Universiteit Eindhoven, The Netherlands \\ c.stahl@tue.nl \\ 3 Institut für Informatik, Humboldt-Universität zu Berlin, Germany \\ richard.mueller@informatik.hu-berlin.de
}

\begin{abstract}
We study open systems modeled as Petri nets with an interface for asynchronous (i.e., buffered) communication with other open systems. As a minimal requirement for successful communication, we investigate responsiveness, which guarantees that an open system and its environment always have the possibility to communicate. We investigate responsiveness with and without final states and also their respective bounded variants, where the number of pending messages never exceeds a previously known bound. Responsiveness accordance describes when one open system can be safely replaced by another open system. We present a trace-based characterization for each accordance variant. As none of the relations turns out to be compositional (i.e., it is no precongruence), we characterize the coarsest compositional relation (i.e., the coarsest precongruence) that is contained in each relation, using a variation of should testing. For the two unbounded variants, the precongruences are not decidable, but for the two bounded variants we show decidability.
\end{abstract}

Keywords: Petri nets, Asynchronous communication, Compositionality, Precongruence, Should testing preorder

\section{Introduction}

Today's software systems are complex distributed systems that are composed of less complex open systems. In this paper, we focus on open systems that have a well-defined interface and communicate with each other via asynchronous message passing. Service-oriented systems like Web-service applications [27] and systems based on wireless network technologies like wireless sensor networks [2, medical systems, transportation systems, or online gaming are examples of such distributed systems. During system evolution, often one open system is replaced by another one-for example, when new features have been implemented or 
bugs have been fixed. This requires a refinement notion, which should respect compositionality.

In this paper, we model an open system as a Petri net extended with an interface for asynchronous communication. As a minimal requirement for successful communication, responsiveness demands that an open system and its environment (called a controller) always have the possibility to communicate. An open system is in responsiveness accordance with another one, if it can replace the latter as part of a closed system without affecting this property. The related property of deadlock freedom can be satisfied by one component that works internally forever. Responsiveness has gained interest because it additionally ensures the possibility to communicate, which is crucial in the setting of interacting open systems. An example is Microsoft's asynchronous event driven programming language $\mathrm{P}$, which is used to implement device drivers [11. $\mathrm{P}$ programs can be checked for a stricter variant of responsiveness, which additionally requires that no message in any channel is ignored forever.

We present a trace-based characterization of responsiveness accordance. The semantics consists of a set of completed traces and responsiveness accordance is characterized by trace inclusion. Usually, controller-based preorders like ours are precongruences and, thus, a compositional refinement notion on open systems; as this is not the case for our responsiveness accordance, we characterize the coarsest precongruence that is contained in this preorder. Interestingly, we obtain Vogler's $\mathcal{F}^{+}$-semantics [33] (which has been later introduced as impossible futures [36]), and the corresponding precongruence is the should testing preorder $25[6 \mid 29]$. Such a characterization is vital, because the definition of a coarsest precongruence considers arbitrary parallel environments and is, therefore, hard to check in concrete cases. Also, a declarative characterization furthers understanding and can, for example, help to decide the precongruence.

In addition, we study responsiveness in the presence of final states. Again, we characterize the preorder based on traces. To distinguish final and nonfinal states we collect successfully and unsuccessfully completed traces. As also this preorder is not a precongruence, we characterize the coarsest precongruence as a variant of should testing. More precisely, we have to add an additional set to the $\mathcal{F}^{+}$-semantics collecting all traces that do not lead to a final state. The precongruence can be defined in line with the should testing preorder.

In unpublished work, we showed that neither the preorders nor the precongruences are decidable. These results are not in the scope of this paper, as the proof technique applied is very different from those we use here. This motivates us to investigate beside final and nonfinal states a second dimension of responsiveness, boundedness, resulting in two additional variants of responsiveness. For these two variants, we require the composition of two open systems to be finitestate and, in particular, that the number of pending messages never exceeds a previously known bound. This is practically relevant: Distributed systems operate on a middleware with buffers that are of bounded size. The actual buffer size can be the result of a static analysis of the underlying middleware or of the communication behavior of an open system, or simply be chosen sufficiently large. 
We give a trace-based characterization for each bounded variant of responsiveness accordance, thereby adapting and combining results from the unbounded variants and work on traces that cannot be used reliably by any controller [21]. Due to the latter traces, accordant systems may violate language inclusion. Giving an answer to an open question, we show again that none of the accordance variants turns out to be a precongruence. So we characterize for each variant its coarsest precongruence that is contained in the preorder. To this end, we add information about bound violations to the precongruence of the respective unbounded variant. In fact, as we require a user to define the upper bound $b$ of pending messages, we obtain a family of preorders and precongruences, each parameterized by $b$. Based on our characterization, we prove the two coarsest precongruences in the bounded case to be decidable: for the first precongruence, the problem can be reduced to deciding should testing [29]; for the second, we refine the proof in 29] by further details.

The goal of this article is to contribute to a general theory on open systems in the presence of an asynchronous unqueued (i.e., buffered) communication scheme. Although we present only the theory, open systems specified in industrial languages such as WS-BPEL or BPMN can be translated into our formal model and then be analyzed 19 .

Our contribution can be summarized as follows:

- We give a trace-based characterization for the four variants of responsiveness accordance.

- For each variant of accordance, we characterize the coarsest precongruence that is contained in the respective preorder as variants of the should testing preorder.

- For the two bounded variants, we show decidability of the precongruences.

This article extends previous work of the same authors [35/34. In [35, we presented an extended abstract of unbounded responsiveness accordance and the respective variant in the presence of final states. In this article, we present these two variants including all proofs. The work in [34, is an extended abstract of the bounded variant of responsiveness accordance without final states and presents only a sketch of the main results of the respective variant in the presence of final states. In this article, we present all the results of the bounded variants including all proofs.

After some background in Sect.2, Sect. 3introduces the basic variant, responsiveness (i.e., with possibly unbounded message buffers and without final states), characterizes the respective accordance relation semantically and presents a characterization of the coarsest precongruence that is contained in this relation. The presence of final states is investigated in Sect. 4. Section 5 prepares for dealing with boundedness and presents a precongruence for boundedness without considering responsiveness. Next, Sect. 6 introduces bounded responsiveness, characterizes the respective accordance relation semantically, presents a characterization of the coarsest precongruence that is contained in this relation, and proves its decidability. Section 7 characterizes bounded responsiveness in the 
presence of final states. We discuss related work in Sect. 8 and close with a conclusion in Sect. 9 .

\section{Preliminaries}

This section provides the basic notions, such as Petri nets, open nets for modeling open systems, and open net environments for describing the semantics of open nets.

For two sets $A$ and $B$, let $A \uplus B$ denote the disjoint union; writing $A \uplus B$ implies that $A$ and $B$ are implicitly assumed to be disjoint. Let $\mathbb{N}$ denote the natural numbers including 0 .

\section{$2.1 \quad$ Petri nets}

As a basic model, we use place/transition Petri nets extended with a set of final markings and transition labels.

Definition 2.1 (net). A net $N=\left(P, T, F, m_{N}, \Omega\right)$ consists of

- a set $P$ of places,

- a set $T$ of transitions such that $P$ and $T$ are disjoint,

- a flow relation $F \subseteq(P \times T) \uplus(T \times P)$,

- an initial marking $m_{N}$, where a marking is a mapping $m: P \rightarrow \mathbb{N}$, and

- a set $\Omega$ of final markings.

Usually, we are interested in finite nets - that is, nets with finite sets $P$ and $T$-but for some results (e.g., Theorems 3.17 and 4.17), we also make use of infinite nets.

Introducing a net $N$ also implicitly introduces its components $P, T, F, m_{N}, \Omega$; the same applies to nets $N^{\prime}, N_{1}$, etc. and their components $P^{\prime}, T^{\prime}, F^{\prime}, m_{N^{\prime}}, \Omega^{\prime}$, and $P_{1}, T_{1}, F_{1}, m_{N_{1}}, \Omega_{1}$, respectively - and it also applies to other structures later on.

Definition 2.2 (labeled net). A labeled net $N=\left(P, T, F, m_{N}, \Omega, \Sigma_{\text {in }}, \Sigma_{\text {out }}, l\right)$ is a net $\left(P, T, F, m_{N}, \Omega\right)$ together with an alphabet $\Sigma=\Sigma_{\text {in }} \uplus \Sigma_{\text {out }}$ of disjoint input actions $\Sigma_{i n}$ and output actions $\Sigma_{\text {out }}$ and a labeling function $l: T \rightarrow$ $\Sigma \uplus\{\tau\}$, where $\tau$ represents an invisible, internal action. Two labeled nets are interface-equivalent if they have the same sets of input and output actions.

Graphically, a circle represents a place, a box represents a transition, and the directed arcs between places and transitions represent the flow relation. In the case of a labeled net, a transition label is depicted inside a transition with bold font to distinguish it from the transition's identity. A marking is a distribution of tokens over the places. Graphically, a black dot represents a token.

Let $x \in P \uplus T$ be a node of a net $N$. As usual, ${ }^{\bullet} x=\{y \mid(y, x) \in F\}$ denotes the preset of $x$ and $x^{\bullet}=\{y \mid(x, y) \in F\}$ the postset of $x$. We interpret presets and postsets as multisets when used in operations also involving multisets. 
A marking is a multiset over the set $P$ of places; for example, $\left[p_{1}, 2 p_{2}\right]$ denotes a marking $m$ with $m\left(p_{1}\right)=1, m\left(p_{2}\right)=2$, and $m(p)=0$ for $p \in P \backslash\left\{p_{1}, p_{2}\right\}$. We define + and - for the sum and the difference of two markings and $=,<,>, \leq, \geq$ for comparison of markings in the standard way. We canonically extend the notion of a marking of $N$ to supersets $Q \supseteq P$ of places; that is, for a mapping $m: P \rightarrow \mathbb{N}$, we extend $m$ to the marking $m: Q \rightarrow \mathbb{N}$ such that for all $p \in Q \backslash P$, $m(p)=0$. Conversely, a marking can be restricted to a subset $Q \subseteq P$ of the places of $N$. For a mapping $m: P \rightarrow \mathbb{N}$, the restriction of $m$ to the places in $Q$ is denoted by $\left.m\right|_{Q}: Q \rightarrow \mathbb{N}$.

Let $\Sigma_{1}, \Sigma_{2}$ be alphabets. For a word $w \in \Sigma_{1}^{*}$ and $\Sigma_{2} \subseteq \Sigma_{1},\left.w\right|_{\Sigma_{2}}$ denotes the projection of $w$ to the subalphabet $\Sigma_{2}$. With $v \sqsubseteq w$ we denote that $v$ is a prefix of $w$. We write $|w|$ for the length of $w$, and $|w|_{x}$ denotes how many times $x \in \Sigma$ occurs in word $w$. As usual, $\varepsilon$ denotes the empty word.

The behavior of a net $N$ relies on the marking of $N$ and changing the marking by the firing of transitions of $N$. A transition $t \in T$ is enabled at a marking $m$, denoted by $m \stackrel{t}{\rightarrow}$, if for all $p \in \bullet, m(p)>0$. If $t$ is enabled at $m$, it can fire, thereby changing the current marking $m$ to a marking $m^{\prime}=m-{ }^{\bullet} t+t^{\bullet}$. The firing of $t$ is denoted by $m \stackrel{t}{\rightarrow} m^{\prime}$; that is, $t$ is enabled at $m$ and firing it results in $m^{\prime}$.

The behavior of $N$ can be extended to sequences: $m_{1} \stackrel{t_{1}}{\longrightarrow} \ldots \stackrel{t_{k-1}}{\longrightarrow} m_{k}$ is a run of $N$ if for all $0<i<k, m_{i} \stackrel{t_{i}}{\rightarrow} m_{i+1}$. A marking $m^{\prime}$ is reachable from a marking $m$ if there exists a (possibly empty) run $m_{1} \stackrel{t_{1}}{\longrightarrow} \ldots \stackrel{t_{k-1}}{\longrightarrow} m_{k}$ with $m=m_{1}$ and $m^{\prime}=m_{k}$; for $v=t_{1} \ldots t_{k-1}$, we also write $m_{1} \stackrel{v}{\rightarrow} m_{k}$. A marking $m^{\prime}$ is reachable if it is reachable from the initial marking. The set $M_{N}$ represents the set of all reachable markings of $N$.

In the case of labeled nets, we lift runs to traces: If $m_{1} \stackrel{v}{\rightarrow} m_{k}$ and $w$ is obtained from $v$ by replacing each transition with its label and removing all $\tau$ labels, we write $m_{1} \stackrel{w}{\Longrightarrow} m_{k}$ and refer to $w$ as a trace whenever $m_{1}=m_{N}$. The language $L(N)$ of a labeled net $N$ is the set of all traces of $N$. The reachability graph $R G(N)$ of $N$ has the reachable markings $M_{N}$ as its nodes and a $t$-labeled edge from $m$ to $m^{\prime}$ whenever $m \stackrel{t}{\rightarrow} m^{\prime}$ in $N$. In the case of a labeled net, each edge label $t$ is replaced with $l(t)$.

Finally, we introduce boundedness of nets. A marking $m$ of a net $N$ is $b$ bounded for a bound $b \in \mathbb{N}$, if $m(p) \leq b$ for all $p \in P$. The net $N$ is $b$-bounded if every reachable marking is $b$-bounded; it is bounded, if it is $b$-bounded for some $b \in \mathbb{N}$. Throughout the paper, $b$ denotes a bound - a positive natural number.

\subsection{Open nets}

Like Lohmann et al. [18 and Stahl et al. [30, we model open systems as open nets 3318, thereby restricting ourselves to the communication protocol of an open system. An open net extends a net by an interface. An interface consists of two disjoint sets of input and output places corresponding to asynchronous input and output channels. In the model, we abstract from data and represent each message by a token on the respective interface place. In the initial marking 
and the final markings, interface places are not marked. An input place has an empty preset, and an output place has an empty postset. We consider only open nets that have either at least one input and one output place or no input and output places; open nets with just input or just output places cannot really take part in a responsive communication.

Definition 2.3 (open net). An open net $N$ is a tuple $\left(P, T, F, m_{N}, I, O, \Omega\right)$ such that

- $\left(P \uplus I \uplus O, T, F, m_{N}, \Omega\right)$ is a net;

- for all $p \in I \uplus O, m_{N}(p)=0$ and for all $m \in \Omega, m(p)=0$;

- the set $I$ of input places satisfies for all $p \in I, \bullet p=\emptyset$;

- the set $O$ of output places satisfies for all $p \in O, p^{\bullet}=\emptyset$; and

- set $I=\emptyset$ if and only if set $O=\emptyset$.

If $I=O=\emptyset$, then $N$ is a closed net. The net inner $(N)$ results from removing the interface places and their incident arcs from $N$. Two open nets are interface equivalent if they have the same sets of input and output places.

Graphically, we represent an open net like a net with a dashed frame around it. An interface place $p$ is positioned on the frame; an additional arrow indicates whether $p$ is an input or an output place.

For the composition of open nets, we assume that the sets of transitions are pairwise disjoint and that no internal place of an open net is a place of any other open net. In contrast, the interfaces intentionally overlap. We require that all communication is bilateral and directed; that is, every shared place $p$ has only one open net that sends into $p$ and one open net that receives from $p$. In addition, we require that either all interface places are shared or there is at least one input and one output place which are not shared. We refer to open nets that fulfill these conditions as composable. We compose two composable open nets $N_{1}$ and $N_{2}$ by merging shared interface places and turning these places into internal places. The definition of composable thereby guarantees that an open net composition is again an open net (possibly a closed net).

Definition 2.4 (open net composition). Two open nets $N_{1}$ and $N_{2}$ are composable if $\left(P_{1} \uplus T_{1} \uplus I_{1} \uplus O_{1}\right) \cap\left(P_{2} \uplus T_{2} \uplus I_{2} \uplus O_{2}\right)=\left(I_{1} \cap O_{2}\right) \uplus\left(I_{2} \cap O_{1}\right)$, and $\left(I_{1} \uplus I_{2}\right) \backslash\left(O_{1} \uplus O_{2}\right)$ and $\left(O_{1} \uplus O_{2}\right) \backslash\left(I_{1} \uplus I_{2}\right)$ are both either empty or nonempty. The composition of two composable open nets $N_{1}$ and $N_{2}$ is the open net $N_{1} \oplus N_{2}=\left(P, T, F, m_{N}, I, O, \Omega\right)$, where

- $P=P_{1} \uplus P_{2} \uplus\left(I_{1} \cap O_{2}\right) \uplus\left(I_{2} \cap O_{1}\right)$,

$-T=T_{1} \uplus T_{2}$,

$-F=F_{1} \uplus F_{2}$,

- $m_{N}=m_{N_{1}}+m_{N_{2}}$,

- I=(I $\left.I_{1} \uplus I_{2}\right) \backslash\left(O_{1} \uplus O_{2}\right)$,

- $O=\left(O_{1} \uplus O_{2}\right) \backslash\left(I_{1} \uplus I_{2}\right)$, and

$-\Omega=\left\{m_{1}+m_{2} \mid m_{1} \in \Omega_{1}, m_{2} \in \Omega_{2}\right\}$. 


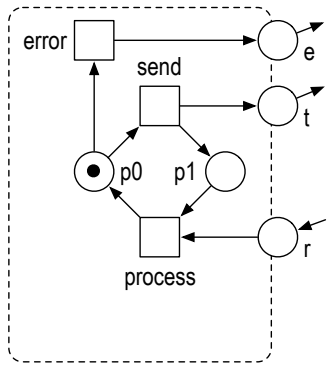

(a) Open net $S$

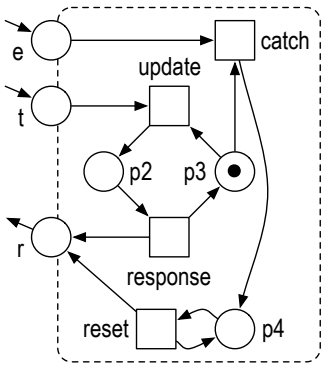

(b) Open net $C$

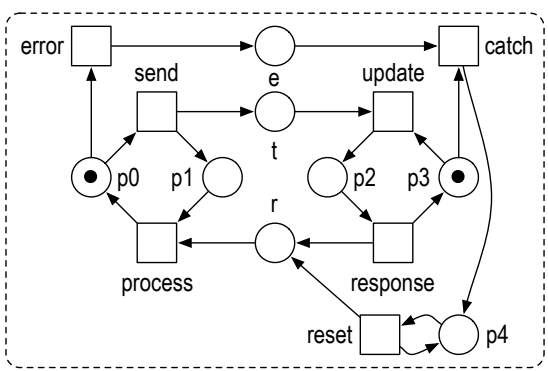

(c) Open net $S \oplus C$

Fig. 1: Open nets modeling an unreliable time server, a client, and their composition. In addition to the models, we have $\Omega_{S}=\{[]\}$ and $\Omega_{C}=\Omega_{S \oplus C}=\left\{\left[p_{3}\right]\right\}$.

Example 2.1. Figure 1 shows three open systems, each modeled as an open net. The open net $S$ models an unreliable time server that sends its timing information (output place $t$ ) to some client and processes its responses (input place $r$ ). Anytime before sending the timing information, an error may happen (output place $e$ ) and the server shuts down (and final marking [] can be reached). The open net $C$ models a client. It repeatedly updates its system time by the timing information sent by the server (input place $t$ ) and responds with a response packet (output place $r$ ). If the client receives an error message from the server (input place $e$ ), it continuously tries to reset the time server (output place $r$ ). The open nets $S$ and $C$ are composable. Their composition $S \oplus C$ is a closed net, which is depicted in Fig. 1c. The place $r$ in $S \oplus C$ is unbounded; thus, the composition is unbounded, too.

\subsection{Environments}

To give an open net $N$ a trace-based semantics, we consider its environment env $(N)$, which we define similarly to Vogler [33. The net env $(N)$ can be constructed from $N$ by adding to each interface place $p \in I(p \in O)$ a $p$-labeled transition $p$ in env $(N)$ and renaming the place $p$ to $p^{i}\left(p^{o}\right)$. The net env $(N)$ 
shows the possible behaviour of an environment of $N$-that is, which inputs it can send to $N$ and which outputs it can receive from $N$. It is just a tool to define our characterizations and prove our results. But intuitively, one can understand the construction as translating the asynchronous interface $p$ of $N$ into a buffered synchronous interface (with unbounded buffers $p^{i}$ or $p^{o}$ ) described by the transition labels of $e n v(N)$.

Definition 2.5 (open net environment). The environment of an open net $N$ is the labeled net env $(N)=\left(P \uplus P^{I} \uplus P^{O}, T \uplus I \uplus O, F^{\prime}, m_{N}, \Omega, I, O, l^{\prime}\right)$, where

$-P^{I}=\left\{p^{i} \mid p \in I\right\}$,

- $P^{O}=\left\{p^{o} \mid p \in O\right\}$,

- $F^{\prime}=((P \uplus T) \times(T \uplus P)) \cap F$

$\uplus\left\{\left(p^{i}, t\right) \mid p \in I, t \in T,(p, t) \in F\right\}$

$\uplus\left\{\left(t, p^{o}\right) \mid p \in O, t \in T,(t, p) \in F\right\}$

$\uplus\left\{\left(p^{o}, p\right) \mid p \in O\right\}$

$\uplus\left\{\left(p, p^{i}\right) \mid p \in I\right\}$, and

$-l^{\prime}(t)= \begin{cases}\tau, & t \in T \\ t, & t \in I \uplus O .\end{cases}$

Convention: Throughout the paper, each trace set and semantics for labeled nets is implicitly extended to any open net $N$ via env $(N)$ - for example, the language of $N$ is defined as $L(N)=L(\operatorname{env}(N))$.

To compose environments of composable open nets in particular and labeled nets in general, we define a parallel composition operator $\|$ where, for each action $a$ that the components have in common, each $a$-labeled transition of one component is synchronized with each $a$-labeled transition of the other. In addition, we define a second parallel composition operator $\Uparrow$. This operator works as operator $\|$ and, in addition, hides all common actions - that is, changes the respective labels to $\tau$. Hiding and $\|$ are defined as in [33.

Definition 2.6 (parallel composition and hiding). Two labeled nets $N_{1}$ and $N_{2}$ are composable if $P_{1} \cap P_{2}=\Sigma_{\text {in } 1} \cap \Sigma_{\text {in } 2}=\Sigma_{\text {out } 1} \cap \Sigma_{\text {out } 2}=\emptyset$. The parallel composition of two composable labeled nets is the labeled net $N_{1} \| N_{2}=$ $\left(P, T, F, m_{N}, \Omega, \Sigma_{\text {in }}, \Sigma_{\text {out }}, l\right)$, where

- $P=P_{1} \uplus P_{2}$,

$-T=\left\{\left(t_{1}, t_{2}\right) \in T_{1} \times T_{2} \mid l_{1}\left(t_{1}\right)=l_{2}\left(t_{2}\right) \neq \tau\right\}$

$\uplus\left\{\left(t_{1}, \tau\right) \in T_{1} \times\{\tau\} \mid l_{1}\left(t_{1}\right) \notin \Sigma_{2}\right\}$

$\uplus\left\{\left(\tau, t_{2}\right) \in\{\tau\} \times T_{2} \mid l_{2}\left(t_{2}\right) \notin \Sigma_{1}\right\}$,

- F $=\left\{\left(p,\left(t_{1}, t_{2}\right)\right) \in P \times T \mid\left(p, t_{1}\right) \in F_{1} \vee\left(p, t_{2}\right) \in F_{2}\right\}$

$\uplus\left\{\left(\left(t_{1}, t_{2}\right), p\right) \in T \times P \mid\left(t_{1}, p\right) \in F_{1} \vee\left(t_{2}, p\right) \in F_{2}\right\}$,

$-m_{N}=m_{N_{1}}+m_{N_{2}}$

$-\Omega=\left\{m_{1}+m_{2} \mid m_{1} \in \Omega_{1}, m_{2} \in \Omega_{2}\right\}$,

$-\Sigma_{\text {in }}=\left(\Sigma_{\text {in } 1} \uplus \Sigma_{\text {in } 2}\right) \backslash\left(\Sigma_{\text {out } 1} \uplus \Sigma_{\text {out } 2}\right)$,

- $\Sigma_{\text {out }}=\Sigma_{\text {out } 1} \uplus \Sigma_{\text {out } 2}$, and 
$-l\left(t_{1}, t_{2}\right)= \begin{cases}l_{1}\left(t_{1}\right), & t_{1} \in T_{1} \\ l_{2}\left(t_{2}\right), & \text { otherwise }\end{cases}$

For a labeled net $N$ and a set $A \subseteq \Sigma$, we obtain $N / A$ from $N$ by hiding all actions of $A$, meaning we replace the respective labels in $A$ with $\tau$. The parallel composition with hiding is the labeled net $N_{1} \Uparrow N_{2}=\left(N_{1} \| N_{2}\right) /\left(\Sigma_{1} \cap \Sigma_{2}\right)$.

Example 2.2. Figure 2 shows the environments of the open nets $S$ and $C$ from Fig. 1, their parallel composition env $(S) \|$ env $(C)$, and their parallel composition with hiding $\operatorname{env}(S) \Uparrow e n v(C)$.

To describe the behavior of compositions, we define parallel compositions of words and languages; operator $\|$ synchronizes common actions, operator $\Uparrow$ also hides them. Observe that in env $\left(N_{1}\right) \Uparrow e n v\left(N_{2}\right)$ only common transitions are merged; operator $\|$ is needed to relate the respective transition sequences.

Definition 2.7. Let $\Sigma_{1}, \Sigma_{2}$ be alphabets and $\Sigma=\left(\Sigma_{1} \cup \Sigma_{2}\right) \backslash\left(\Sigma_{1} \cap \Sigma_{2}\right)$. Let $w_{1} \in \Sigma_{1}^{*}$ and $w_{2} \in \Sigma_{2}^{*}$ be words, and let $L_{1} \subseteq \Sigma_{1}^{*}$ and $L_{2} \subseteq \Sigma_{2}^{*}$ be languages. We define 4

$-w_{1} \| w_{2}=\left\{w \in\left(\Sigma_{1} \cup \Sigma_{2}\right)^{*}|w|_{\Sigma_{1}}=w_{1},\left.w\right|_{\Sigma_{2}}=w_{2}\right\}$,

- $w_{1} \Uparrow w_{2}=\left\{\left.w\right|_{\Sigma} \mid w \in w_{1} \| w_{2}\right\}$,

- $L_{1} \| L_{2}=\bigcup\left\{w_{1} \| w_{2} \mid w_{1} \in L_{1}, w_{2} \in L_{2}\right\}$, and

- $L_{1} \Uparrow L_{2}=\bigcup\left\{w_{1} \Uparrow w_{2} \mid w_{1} \in L_{1}, w_{2} \in L_{2}\right\}$.

The next proposition recalls [33, Theorem 3.1.7(4)] and relates a trace of a composed labeled net to traces of its components.

Proposition 2.8 ([33]). For two markings $m_{1}$ and $m_{2}$ of composable labeled nets $N_{1}$ and $N_{2}$, respectively, we have $m_{1}+m_{2} \stackrel{w}{\Longrightarrow} m_{1}^{\prime}+m_{2}^{\prime}$ in $N_{1} \| N_{2}$ iff there exist $w_{1} \in \Sigma_{N_{1}}^{*}, w_{2} \in \Sigma_{N_{2}}^{*}$ such that $w \in w_{1} \| w_{2}, m_{1} \stackrel{w_{1}}{\Longrightarrow} m_{1}^{\prime}$ in $N_{1}$, and $m_{2} \stackrel{w_{2}}{\Longrightarrow} m_{2}^{\prime}$ in $N_{2}$.

For the remainder of this section, we fix two composable open nets $N_{1}$ and $N_{2}$, and we put $C=\operatorname{env}\left(N_{1} \oplus N_{2}\right), E=e n v\left(N_{1}\right) \| e n v\left(N_{2}\right)$, and $\bar{E}=\operatorname{env}\left(N_{1}\right) \Uparrow$ env $\left(N_{2}\right)$. The labeled nets $\bar{E}$ and $E$ differ only in their labelings; $C$ and $\bar{E}$ $(E)$ have the same places, except for places $p \in\left(I_{1} \cap O_{2}\right) \uplus\left(I_{2} \cap O_{1}\right)$ in $C$ and the corresponding places $p^{i}, p^{o}$ in $\bar{E}(E)$. We study the relation between reachable markings of different compositions of $N_{1}$ and $N_{2}$. To this end, we define agreement between markings.

Definition 2.9 (agreement). A marking $m$ of $C$ and a marking $\bar{m}$ of $\bar{E}$ (of $E$ ) agree if they coincide on the common places and if for each $p \in\left(I_{1} \cap O_{2}\right) \uplus\left(I_{2} \cap\right.$ $\left.O_{1}\right), \bar{m}\left(p^{o}\right)+\bar{m}\left(p^{i}\right)=m(p)$. They strongly agree if, additionally, $\bar{m}\left(p^{o}\right)=0$.

\footnotetext{
${ }^{4}$ To simplify the notation, we do not add the alphabets of $w_{1}$ and $w_{2}$ to operators $\|$ and $\Uparrow$; the alphabets will be always clear from the context.
} 


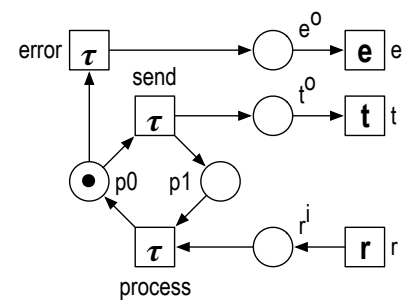

(a) Labeled net $\operatorname{env}(S)$

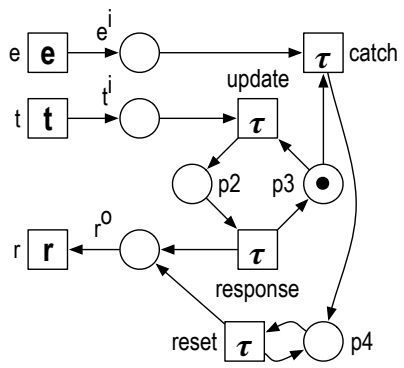

(b) Labeled net $\operatorname{env}(C)$

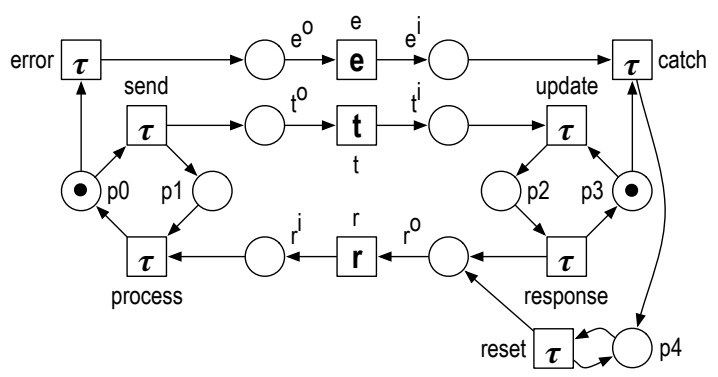

(c) Labeled net env $(S) \| \operatorname{env}(C)$

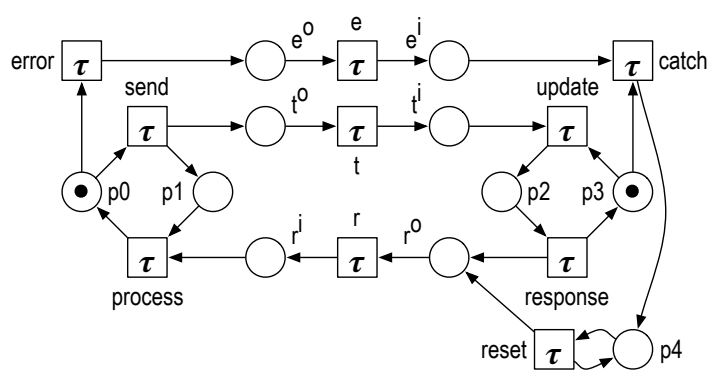

(d) Labeled net $\operatorname{env}(S) \Uparrow e n v(C)$

Fig. 2: The environments of the time server and the client and their parallel compositions. In addition to the models, we have $\Omega_{e n v(S)}=\{[]\}, \Omega_{e n v(C)}=$ $\Omega_{e n v(S) \| e n v(C)}=\Omega_{e n v(S) \Uparrow e n v(C)}=\left\{\left[p_{3}\right]\right\}$.

The next proposition recalls [32, Lemma 15] and relates the firing of a transition of $C$ and $\bar{E}(E)$.

Proposition 2.10 ([32]). Let the markings $m$ of $C$ and $\bar{m}$ of $\bar{E}$ agree, and let $t \in T_{C}$. Then

1. If $\bar{m} \stackrel{t}{\rightarrow} \bar{m}^{\prime}$, then $m \stackrel{t}{\rightarrow} m^{\prime}$ such that $m^{\prime}$ and $\bar{m}^{\prime}$ agree. 
2. If $m$ and $\bar{m}$ strongly agree, all additional transitions of $\bar{E}$ are disabled at $\bar{m}$, and further $m \stackrel{t}{\rightarrow} m^{\prime}$ implies $\bar{m} \stackrel{t}{\rightarrow} \bar{m}^{\prime}$ such that $m^{\prime}$ and $\bar{m}^{\prime}$ agree.

As $\bar{E}$ and $E$ differ only in their labelings, (1)-(2) also hold for $E$ in place of $\bar{E}$.

The next proposition recalls [32, Lemma 16(1),(2)] and [32, Lemma 15(4)]. It proves facts about sequences of transitions and relates final states.

Proposition 2.11 ([32]). Let $m$ be a marking of $C$ and $\bar{m}$ be one of $\bar{E}$. Then

1. If $m$ and $\bar{m}$ strongly agree and $m \stackrel{v}{\rightarrow} m^{\prime}$ in $C$, then it is possible to insert transitions from $\left(I_{1} \cap O_{2}\right) \uplus\left(I_{2} \cap O_{1}\right)$ of $\bar{E}$ into $v$ such that for the resulting $v^{\prime}: \bar{m} \stackrel{v^{\prime}}{\rightarrow} \bar{m}^{\prime}$ in $\bar{E}$ and also $m^{\prime}$ and $\bar{m}^{\prime}$ strongly agree.

2. If $m$ and $\bar{m}$ agree and $\bar{m} \stackrel{v^{\prime}}{\rightarrow} \bar{m}^{\prime}$ in $\bar{E}$, then it is possible to delete transitions from $\left(I_{1} \cap O_{2}\right) \uplus\left(I_{2} \cap O_{1}\right)$ of $\bar{E}$ in $v^{\prime}$ such that for the resulting $v: m \stackrel{v}{\rightarrow} m^{\prime}$ in $C$ and also $m^{\prime}$ and $\bar{m}^{\prime}$ agree.

3. $m \in \Omega_{C}$ iff $\bar{m} \in \Omega_{\bar{E}}$ iff for $i=1,2:\left.\bar{m}\right|_{P_{e n v\left(N_{i}\right)}} \in \Omega_{e n v\left(N_{i}\right)}$.

$A s \bar{E}$ and $E$ differ only in their labelings, (1)-(3) also hold for $E$ in place of $\bar{E}$.

Agreement between markings of $C$ and $\bar{E}$ is a weak bisimulation [22].

Lemma 2.12. The labeled nets $C$ and $\bar{E}$ are weakly bisimilar due to the agreement relation.

Proof. First, we show that if we label each transition of $C$ with itself in $C$ and $\bar{E}$ and every transition $t \in\left(I_{1} \backslash O_{2}\right) \uplus\left(I_{2} \backslash O_{1}\right)$ in $\bar{E}$ with $\tau$, then agreement between the markings of $C$ and $\bar{E}$ is a weak bisimulation.

The initial markings $m_{C}$ and $m_{\bar{E}}$ strongly agree by Definitions 2.4 and 2.5 . Writing $\varrho$ for the agreement relation, we now assume that $(m, \bar{m}) \in \varrho$. To prove that $\varrho$ is a weak bisimulation, we have to show that

1. If $m \stackrel{t}{\rightarrow} m^{\prime}$, then there exists $\bar{m}^{\prime}$ such that $\bar{m} \stackrel{t}{\Rightarrow} \bar{m}^{\prime}$ and $\left(m^{\prime}, \bar{m}^{\prime}\right) \in \varrho$; and

2. If $\bar{m} \stackrel{t}{\rightarrow} \bar{m}^{\prime}$, then there exists $m^{\prime}$ such that $m \stackrel{t}{\Rightarrow} m^{\prime}$ and $\left(m^{\prime}, \bar{m}^{\prime}\right) \in \varrho$.

Consider the first item. By firing all $\tau$-labeled transitions of $\bar{E}$ that are enabled at $\bar{m}$, we can empty each place $p^{o}$ while shifting the tokens to the respective place $p^{i}$. Let $\bar{m}^{\prime \prime}$ be the resulting marking of $\bar{E}$. Then $\bar{m} \stackrel{\varepsilon}{\Longrightarrow} \bar{m}^{\prime \prime}$ and $\bar{m}^{\prime \prime}$ strongly agrees with $m$, because firing a $\tau$-labeled transition does not change the marking on the common places and no place $p^{o}$ is marked now. By Proposition 2.10(2), we have $\bar{m}^{\prime \prime} \stackrel{t}{\rightarrow} \bar{m}^{\prime}$ such that $m^{\prime}$ and $\bar{m}^{\prime}$ agree.

For the second item, we can set $m=m^{\prime}$ if $t$ is $\tau$-labeled and, clearly, $m^{\prime}$ and $\bar{m}^{\prime}$ agree then. Otherwise, we can conclude from Proposition 2.10(1) that marking $m^{\prime}$ exists such that $m^{\prime}$ and $\bar{m}^{\prime}$ agree. Thus, $m \stackrel{t}{\Longrightarrow} m^{\prime}$ and $\varrho$ is a weak bisimulation.

Because the original labelings can be obtained by the same relabeling from the labelings considered in the first part of the proof, agreement is also a weak bisimulation for $C$ and $\bar{E}$. 
We introduce a few, more compact notations. Given a set of traces, we define the prefix closure, suffix closure, and the remainder of this set. The suffix closure is also called the set of all continuations of a language.

Definition 2.13 (closures, remainder). Let $U \in \mathcal{P}\left(\Sigma^{*}\right)$. Then,

$-\downarrow U=\left\{u \in \Sigma^{*} \mid \exists v \in U: u \sqsubseteq v\right\}$ is the prefix closure of $U$.

- $U=\left\{u \in \Sigma^{*} \mid \exists v \in U: v \sqsubseteq u\right\}$ is the suffix closure of $U$.

$-v^{-1} U=\left\{u \in \Sigma^{*} \mid v u \in U\right\}$ is the remainder of $v$ in $U$.

For the suffix closure, we can show the following properties.

Lemma 2.14. Let $X, Y \in \mathcal{P}\left(\Sigma^{*}\right)$. Then the following properties hold:

$$
\begin{aligned}
& \text { 1. } \uparrow(X \cup Y)=\uparrow X \cup \uparrow Y \\
& \text { 2. } x^{-1}(X \cup Y)=x^{-1} X \cup x^{-1} Y \\
& \text { 3. } y \notin \uparrow Y \text { implies } y^{-1}(X \cup \uparrow Y) \subseteq \uparrow\left(y^{-1}(X \cup Y)\right)
\end{aligned}
$$

Proof. Items (1) and (2) are trivial. For (3) observe that $y^{-1}(X \cup \uparrow Y)=$ $y^{-1} X \cup y^{-1 \uparrow} Y \subseteq \uparrow\left(y^{-1} X\right) \cup \uparrow\left(y^{-1} Y\right)=\uparrow\left(y^{-1}(X \cup Y)\right)$.

\section{Unbounded nets and no final markings}

In this section, we consider possibly unbounded open nets and ignore final markings. The resulting notions of responsiveness and $r$-accordance yield an equivalence, which is similar to $P$-deadlock equivalence in 33 .

Definition 3.1 (responsiveness). Let $N_{1}$ and $N_{2}$ be composable open nets. A marking $m$ of $N_{1} \oplus N_{2}$ is responsive if we can reach from $m$ a marking that enables a transition $t$ with $t^{\bullet} \cap\left(O_{1} \uplus O_{2}\right) \neq \emptyset$. The open nets $N_{1}$ and $N_{2}$ are responsive if their composition $N_{1} \oplus N_{2}$ is a closed net and every reachable marking of $N_{1} \oplus N_{2}$ is responsive.

Responsiveness ensures that at least one net can talk to the other repeatedly. This property depends on $N_{1}$ and $N_{2}$ in combination: In the composition, each of them will usually not reach all markings it could reach in other contexts; also, it suffices that just one component can enable an output transition. We are actually aiming at a setting with bounded open nets for which the term 'responsive' will imply mutual communication, see Sect. 6 for details.

Based on the correctness criterion responsiveness, we define an $r$-controller of an open net $N$ as an open net $C$ such that $N$ and $C$ are responsive.

Definition 3.2 ( $r$-controller). An open net $C$ is an $r$-controller of an open net $N$ if $N$ and $C$ are responsive.

For every 'truly' (i.e., not closed) open net $N$, there exists an $r$-controllerthe latter just has to continuously send a message, which is possible, because by Definition $2.3 N$ has at least one input place. 


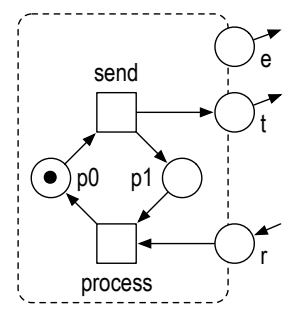

Fig. 3: The open net $S^{\prime}$ modeling a patched time server. We have $\Omega_{S^{\prime}}=\{[]\}$.

If the $r$-controllers of an open net are a superset of the $r$-controllers of another open net, then the first open net is a refinement of the second; intuitively, it makes more users happy due to responsive interaction than the latter. We refer to the resulting refinement relation as $r$-accordance, which gives a necessary requirement for a refinement. For modular reasoning, a refinement relation should be a precongruence for composition. Because $r$-accordance shall turn out not to be one, we will make it stricter (smaller) as far as needed to obtain such a precongruence, and we already introduce a notation for this coarsest precongruence.

Definition 3.3 ( $r$-accordance). For interface-equivalent open nets $I m p l$ and Spec, Impl r-accords with Spec, denoted by Impl $\sqsubseteq_{r, a c c}$ Spec, if for all open nets $C$ the following holds: If $C$ is an $r$-controller of Spec, then $C$ is also an $r$-controller of Impl.

We denote the coarsest precongruence w.r.t. $\oplus$ contained in $\sqsubseteq_{r, a c c}$ by $\sqsubseteq_{r, a c c}^{c}$.

Example 3.1. In Fig. 1 the open net $S$ is an $r$-controller of the open net $C$, and vice versa: Either they can mutually communicate over the interface places $t$ and $r$ or $C$ repeatedly produces a token on place $r$ after consuming a token from $e$. The open net $S^{\prime}$ in Fig. 3 models a patched time server. It has the same functionality as the open net $S$, but it never sends an error message. The open net $S^{\prime} r$-accords with the open net $S$ : Every $r$-controller $C$ of $S$ must expect an error from $S$ (i.e., a token on interface place $e$ ) and, thus, $C$ is also an $r$-controller of $S^{\prime}$, where an error may never happen.

For an example that accordance does not guarantee compositionality, see Fig. 4. Although $S^{\prime} r$-accords with $S, S^{\prime} \oplus A$ does not $r$-accord with $S \oplus A$ : The open net $B$ is an $r$-controller of $S \oplus A$ but not of $S^{\prime} \oplus A$, because the transition catch in $S^{\prime} \oplus A$ can never fire and, thus, firing transition $t_{2}$ in $B$ leads to a nonresponsive marking of $\left(S^{\prime} \oplus A\right) \oplus B$.

In the following, we first give a trace-based characterization for $r$-accordance. Afterward, we characterize the coarsest precongruence that is contained in the $r$-accordance relation. 


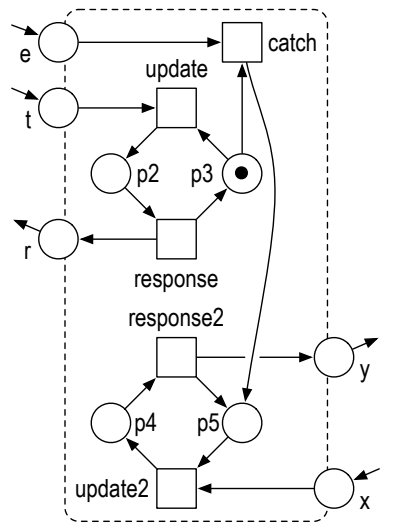

(a) Open net $A$

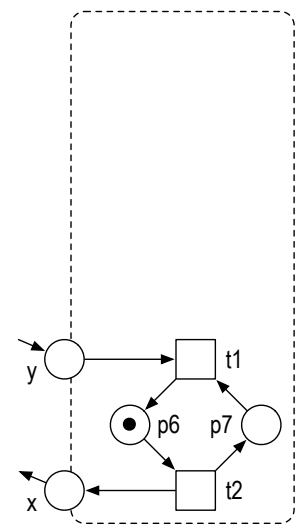

(b) Open net $B$

Fig. 4: Two open nets proving that $r$-accordance (and $f r$-accordance, see Sect. 4 ) is not a precongruence with regard to open net composition $\oplus$. In addition to the models, we have $\Omega_{A}=\left\{\left[p_{5}\right]\right\}$ and $\Omega_{B}=\left\{\left[p_{6}\right]\right\}$.

\subsection{A trace-based semantics for responsiveness}

Our trace-based semantics of an open net $N$ considers the set of stop-traces of its environment $e n v(N)$. A stop-trace records a run of $e n v(N)$ that ends in a marking weakly enabling actions of $I$ only, such that $N$ stops unless some input is provided. This trace is a weak version of a notion with the same name in [3132], where only transitions of $I$ and no $\tau$-transitions are allowed to be enabled.

Definition 3.4 (stop-semantics). Let $N$ be a labeled net. A marking $m$ of $N$ is a stop except for inputs if there is no $o \in \Sigma_{\text {out }}$ such that $m \stackrel{o}{\Longrightarrow}$. The stop-semantics of $N$ is defined by the set of traces

$$
\operatorname{stop}(N)=\left\{w \mid m_{N} \stackrel{w}{\Longrightarrow} m \text { and } m \text { is a stop except for inputs }\right\} .
$$

Example 3.2. Consider the open nets $S$ and $C$ in Fig. 1. The language of $S$ is $L(S)=\left\{\left.w \in\{r, t\}^{*}|\forall u \sqsubseteq w:| u\right|_{t} \leq|u|_{r}+1\right\}$

$$
\cup\left\{\left.w e v\left|w, v \in\{r, t\}^{*} \wedge \forall u \sqsubseteq w:\right| u\right|_{t} \leq|u|_{r}+1 \wedge|w v|_{t} \leq|w|_{r}\right\}
$$

Observe that after firing $e$, transition $r$ is continuously enabled in $\operatorname{env}(S)$ while transition $t$ may also fire because of pending tokens on the place $t^{o}$. Every stoptrace of $S$ either contains an $e$ or the number of $r$ 's is smaller than the number of $t$ 's; more precisely,

$$
\begin{aligned}
\operatorname{stop}(S)= & \left\{\left.w \in\{r, t\}^{*}|| w\right|_{t}=|w|_{r}+1 \wedge \forall u \sqsubseteq w:|u|_{t} \leq|u|_{r}+1\right\} \\
\cup\{w e v \mid w e v \in L(S)\} &
\end{aligned}
$$

For $C$, every stop-trace has an equal number of $t$ 's and $r$ 's, and no stop-trace contains an $e$ because of transition reset; more precisely, $\operatorname{stop}(C)=\left\{\left.w \in\{r, t\}^{*}|| w\right|_{t}=|w|_{r} \wedge \forall v \sqsubseteq w:|v|_{t} \geq|v|_{r}\right\}$. 
The presence of stop-traces in two open nets $N_{1}$ and $N_{2}$ is closely related to the question whether $N_{1}$ and $N_{2}$ are responsive. We first relate responsive markings and stops except for inputs.

Lemma 3.5 (responsive marking vs. stop except for inputs). Let $N_{1}$ and $N_{2}$ be composable open nets such that $N_{1} \oplus N_{2}$ is a closed net, and let $E=\operatorname{env}\left(N_{1}\right) \| e n v\left(N_{2}\right)$. Let $m$ be a marking of $N_{1} \oplus N_{2}$ and $\bar{m}$ be a marking of $E$ such that $m$ and $\bar{m}$ agree. Then the following hold:

1. If $m$ is responsive, then $\bar{m}$ is not a stop except for inputs.

2. If $m$ and $\bar{m}$ strongly agree, the converse of (1) also holds.

Proof. We have $N_{1} \oplus N_{2}=\operatorname{env}\left(N_{1} \oplus N_{2}\right)$ and $\left(I_{1} \cap O_{2}\right) \uplus\left(I_{2} \cap O_{1}\right)=O_{1} \uplus O_{2}$ because $N_{1} \oplus N_{2}$ is a closed net. Let $C=N_{1} \oplus N_{2}$ and $O=O_{1} \uplus O_{2}$.

$\Rightarrow$ : W.l.o.g., assume that $m$ and $\bar{m}$ strongly agree; otherwise, $\bar{m}$ is no stop except for inputs as there exists an $o \in O$ with $\bar{m} \stackrel{o}{\rightarrow}$ by Definition 2.9 , hence $\bar{m} \stackrel{o}{\Longrightarrow}$. As $m$ is responsive, we can fire some $v t$ in $C$ such that $t$ is the first transition produces a token on some $x \in O$, i.e., $m \stackrel{v t}{\longrightarrow} m^{\prime}$ in $C$. Then it is possible to insert transitions from $O$ of $E$ into $v$ such that for the resulting $v^{\prime} t$ : $\bar{m} \stackrel{v^{\prime} t}{\longrightarrow} \bar{m}^{\prime}$ in $E$ and also $m^{\prime}$ and $\bar{m}^{\prime}$ strongly agree by Proposition 2.11.(1). Hence either $\bar{m} \stackrel{y}{\Longrightarrow}$ for one of the inserted transitions $y$ or $\bar{m} \stackrel{x}{\Longrightarrow}$, and $\bar{m}$ is not a stop except for inputs.

$\Leftarrow$ : Because $\bar{m}$ is no stop except for inputs but does not enable any transition $x \in O$ (by strong agreement), we have $\bar{m} \stackrel{v}{\rightarrow} \bar{m}^{\prime} \stackrel{t}{\rightarrow} \bar{m}^{\prime \prime}$ in $E$ where neither $t$ nor any transition in $v$ is in $O$, and $\bar{m}^{\prime \prime}$ enables a transition $x \in O$ disabled at $\bar{m}^{\prime}$. Hence, $x^{o} \in t^{\bullet}$ in $E$ and, consequently, $x \in t^{\bullet}$ in $C$ by Definition 2.5. Applying Proposition 2.11 (2), we get $m \stackrel{v}{\rightarrow} m^{\prime}$ in $C$ such that $m^{\prime}$ and $\bar{m}^{\prime}$ agree. Thus, transition $t$ is enabled at $m^{\prime}$ in $C$, and $m$ is responsive.

Next, we relate a stop except for inputs in the parallel composition of two environments to a stop except for inputs in one of the involved environments.

Lemma 3.6 (stop except for inputs vs. stop-semantics). Let $N_{1}$ and $N_{2}$ be composable open nets, and let $E=\operatorname{env}\left(N_{1}\right) \| \operatorname{env}\left(N_{2}\right)$. Let $m_{1}$ and $m_{2}$ be markings of env $\left(N_{1}\right)$ and env $\left(N_{2}\right)$, respectively. Then, $m=m_{1}+m_{2}$ is a stop except for inputs in $E$ iff $m_{1}$ and $m_{2}$ are stops except for inputs.

Proof. $\Rightarrow$ : W.l.o.g., assume that $m_{1}$ is not a stop except for inputs due to $m_{1} \stackrel{o}{\Longrightarrow}$ with $o \in O_{1}$. As $m_{2}$ enables $o \in I_{2}$, we get $m \stackrel{o}{\Longrightarrow}$ with $o \in O_{1} \uplus O_{2}$ by Proposition 2.8 hence $m$ is no stop except for inputs.

$\Leftarrow$ : Because $m_{1}$ and $m_{2}$ are stops except for inputs, there is no $o \in O_{1} \uplus O_{2}$ such that $m_{1} \stackrel{o}{\Longrightarrow}$ in $e n v\left(N_{1}\right)$ and $m_{2} \stackrel{o}{\Longrightarrow}$ in env $\left(N_{2}\right)$. Applying Proposition 2.8 . $m_{1}+m_{2} \stackrel{o}{\Longrightarrow}$ is not in $E$; thus, $m$ is a stop except for inputs.

The following proposition combines Lemma 3.5 and Lemma 3.6 thereby relating responsiveness and the stop-semantics. 
Proposition 3.7 (responsiveness vs. stop-semantics). Let $N_{1}$ and $N_{2}$ be composable open nets such that $N_{1} \oplus N_{2}$ is a closed net. Then

$$
N_{1} \text { and } N_{2} \text { are responsive iff } \operatorname{stop}\left(N_{1}\right) \cap \operatorname{stop}\left(N_{2}\right)=\emptyset .
$$

Proof. Let $C=N_{1} \oplus N_{2}$ and $E=e n v\left(N_{1}\right) \| \operatorname{env}\left(N_{2}\right)$.

$\Rightarrow$ : Proof by contraposition. Assume a trace $w \in \operatorname{stop}\left(N_{1}\right) \cap \operatorname{stop}\left(N_{2}\right)$. Hence, $m_{e n v\left(N_{1}\right)} \stackrel{w}{\Longrightarrow} m_{1}$ in $\operatorname{env}\left(N_{1}\right)$ and $m_{e n v\left(N_{2}\right)} \stackrel{w}{\Longrightarrow} m_{2}$ in $\operatorname{env}\left(N_{2}\right)$ such that both $m_{1}$ and $m_{2}$ are stops except for inputs. Applying Proposition 2.8, we have $m_{E} \stackrel{w}{\Longrightarrow} m_{1}+m_{2}$ in $E$ and, by Lemma 3.6. $m_{1}+m_{2}$ is a stop except for inputs. Markings $m_{E}$ and $m_{C}$ strongly agree by Definitions 2.4 and 2.5. By Proposition 2.11 (2), a marking $m$ is reachable from $m_{C}$ in $C$ such that $m_{1}+m_{2}$ and $m$ agree, and, by Lemma 3.5. $m$ is not responsive. Thus, $N_{1}$ and $N_{2}$ are not responsive.

$\Leftarrow$ : Proof by contraposition. Assume a marking $m$ is reachable in $C$ such that $m$ is not responsive. Markings $m_{E}$ and $m_{C}$ strongly agree by Definitions 2.4 and 2.5. Applying Proposition 2.11(1), we have $m_{E} \stackrel{w}{\Longrightarrow} m_{1}+m_{2}$ in $E$ for some $w$ such that $m$ and $m_{1}+m_{2}$ strongly agree, and, by Lemma 3.5, $m_{1}+m_{2}$ is a stop

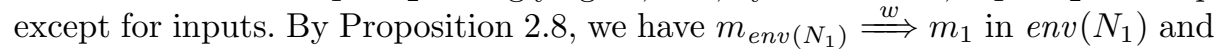
$m_{\text {env }\left(N_{2}\right)} \stackrel{w}{\Longrightarrow} m_{2}$ in $\operatorname{env}\left(N_{2}\right)$, and, by Lemma $3.6, m_{1}$ and $m_{2}$ are stops except for inputs. Thus, $w \in \operatorname{stop}\left(N_{1}\right) \cap \operatorname{stop}\left(N_{2}\right)$.

Example 3.3. For the open nets $S$ and $C$ in Fig. 1 the stop-traces are given in Example 3.2 . One can see that $\operatorname{stop}(S) \cap \operatorname{stop}(C)=\emptyset$; thus, $S$ and $C$ are indeed responsive, as already claimed in Example 3.1 .

The next theorem provides a trace-based characterization of $r$-accordance as inclusion of stop-traces.

Theorem 3.8 ( $r$-accordance and stop inclusion coincide). For two interfaceequivalent open nets Impl and Spec, we have

$$
\operatorname{Impl} \sqsubseteq_{r, a c c} \text { Spec } \quad \text { iff } \quad \text { stop }(\operatorname{Impl}) \subseteq \operatorname{stop}(\text { Spec }) .
$$

Proof. $\Leftarrow$ : Proof by contraposition. Consider an open net $C$ such that $\operatorname{Impl} \oplus C$ and, by interface equivalence, Spec $\oplus C$ are closed nets. Assume that $C$ is not an $r$-controller of Impl. Then Impl and $C$ are not responsive by Definition 3.2, and there exists a trace $w \in \operatorname{stop}(\operatorname{Impl}) \cap \operatorname{stop}(C)$ by Proposition 3.7. Because of stop-inclusion, we have $w \in \operatorname{stop}($ Spec $) \cap \operatorname{stop}(C)$. Again with Proposition 3.7. we see that Spec and $C$ are not responsive; that is, $C$ is not an $r$-controller of Spec.

$\Rightarrow$ : The idea is to construct for a stop-trace $w$ of Impl a net and show using the accordance of $I m p l$ and Spec that $w$ is also a stop-trace of Spec.

Let $I$ be the input and $O$ be the output places of Impl and of Spec. Let $w \in \operatorname{stop}(\operatorname{Impl})$ and $w=w_{1} \ldots w_{n}$ with $w_{j} \in I \uplus O$, for $j=1, \ldots, n$. Define the open net $N_{w}=\left(P, T, F, m_{0}, O, I, \emptyset\right)$ by

$$
\text { - } P=\left\{p_{0}, \ldots, p_{n}\right\},
$$


$-T=\left\{t_{1}, \ldots, t_{n}\right\}$,

$-F=\left\{\left(p_{i}, t_{i+1}\right) \mid 0 \leq i \leq n-1\right\}$

$\uplus\left\{\left(t_{i}, p_{i}\right) \mid 1 \leq i \leq n\right\}$

$\uplus\left\{\left(w_{i}, t_{i}\right) \mid 1 \leq i \leq n, w_{i} \in O\right\}$

$\uplus\left\{\left(t_{i}, w_{i}\right) \mid 1 \leq i \leq n, w_{i} \in I\right\}$, and

$-m_{0}=\left[p_{0}\right]$.

W.l.o.g., we assume $I \neq \emptyset$. Otherwise, $I=O=\emptyset$ by Definition 2.3 and, therefore, stop $(\operatorname{Impl})=\{\varepsilon\} \subseteq\{\varepsilon\}=\operatorname{stop}($ Spec $)$ by Definitions 2.5 and 3.4

Let $o \in I$ be arbitrary but fixed. We extend $N_{w}$ to an open net $N_{w, o}=$ $\left(P^{\prime}, T^{\prime}, F^{\prime}, m_{0}^{\prime}, O, I, \Omega\right)$ - see Fig. 5 - with

$$
\begin{aligned}
-P^{\prime}= & P \uplus\left\{p, p^{\prime}\right\} \uplus\left\{p_{0}^{\prime}, \ldots, p_{n-1}^{\prime}\right\}, \\
-T^{\prime}= & T \uplus\left\{t, t_{0}^{\prime}, \ldots, t_{n-1}^{\prime}, t_{0}^{\prime \prime}, \ldots, t_{n-1}^{\prime \prime}\right\} \uplus\left\{t_{w_{i}} \mid w_{i} \in O\right\}, \\
-F^{\prime}= & F \\
& \uplus\left\{\left(p^{\prime}, t\right),\left(t, p^{\prime}\right),(t, o)\right\} \\
& \uplus\left\{\left(p, t_{w_{i}}\right) \mid w_{i} \in O\right\} \\
& \uplus\left\{\left(t_{w_{i}}, p^{\prime}\right) \mid w_{i} \in O\right\} \\
& \uplus\left\{\left(w_{i}, t_{w_{i}}\right) \mid w_{i} \in O\right\} \\
& \uplus\left\{\left(p_{i}, t_{i}^{\prime}\right) \mid 0 \leq i \leq n-1\right\} \\
& \uplus\left\{\left(t_{i}^{\prime}, p_{i}^{\prime}\right) \mid 0 \leq i \leq n-1\right\} \\
& \uplus\left\{\left(p_{i}^{\prime}, t_{i}^{\prime \prime}\right) \mid 0 \leq i \leq n-1\right\} \\
& \uplus\left\{\left(t_{i}^{\prime}, p_{i}^{\prime}\right) \mid 0 \leq i \leq n-1\right\} \\
& \uplus\left\{\left(t_{i}^{\prime \prime}, o\right) \mid 0 \leq i \leq n-1\right\}, \\
-m_{0}^{\prime}= & {\left[p_{0}, p\right], \text { and } } \\
-\Omega= & \left\{\left[p_{n}, p\right]\right\} .
\end{aligned}
$$

At a stop except for inputs of $\operatorname{env}\left(N_{w, o}\right)$, no transition $t$ with $o \in t^{\bullet}$ (in $\left.N_{w, o}\right)$ is enabled or can be enabled by firing $\tau$-labeled transitions of $\operatorname{env}\left(N_{w, o}\right)$ by Definition 3.4. Hence, a marking of $\operatorname{env}\left(N_{w, o}\right)$ is a reachable stop except for inputs if and only if it is the marking $\left[p_{n}, p\right](1)$ - keep in mind that every $a \in I$ is an output place of $N_{w, o}$.

Obviously, $\operatorname{Impl} \oplus N_{w, o}$ as well as $\operatorname{Spec} \oplus N_{w, o}$ are closed nets by construction of $N_{w, o}$. Because $w \in \operatorname{stop}\left(N_{w, o}\right)$ according to observation (1), Impl and $N_{w, o}$ are not responsive by Proposition 3.7 and choice of $w$. Hence, $N_{w, o}$ is not a controller of Impl by Definition 3.2 and neither a controller of Spec, as Impl accords with Spec. We conclude that Spec and $N_{w, o}$ are not responsive because of Definition 3.2 Again with Proposition 3.7 and Definition 3.4 , there exists a $v \in(I \uplus O)^{*}$ such that

$$
m_{\text {env }(\text { Spec })} \stackrel{v}{\Longrightarrow} m_{1} \text { and } m_{\text {env }\left(N_{w, o}\right)} \stackrel{v}{\Longrightarrow} m_{2},
$$

where both $m_{1}$ and $m_{2}$ are stops except for inputs.

According to observation (1), transitions $t_{1}, \ldots, t_{n}$ of $N_{w, o}$ occur in this order in a run $u$ of $\operatorname{env}\left(N_{w, o}\right)$ underlying $v$ and, thus, there is no occurrence of a transition $t_{j}^{\prime}$ in $u$ by construction. Furthermore, no transition $t_{w_{i}}$ has fired and 


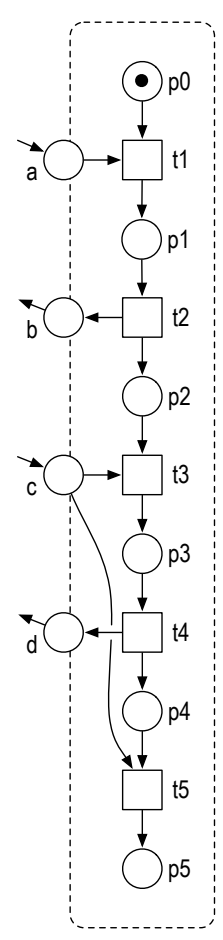

(a) $N_{w}$

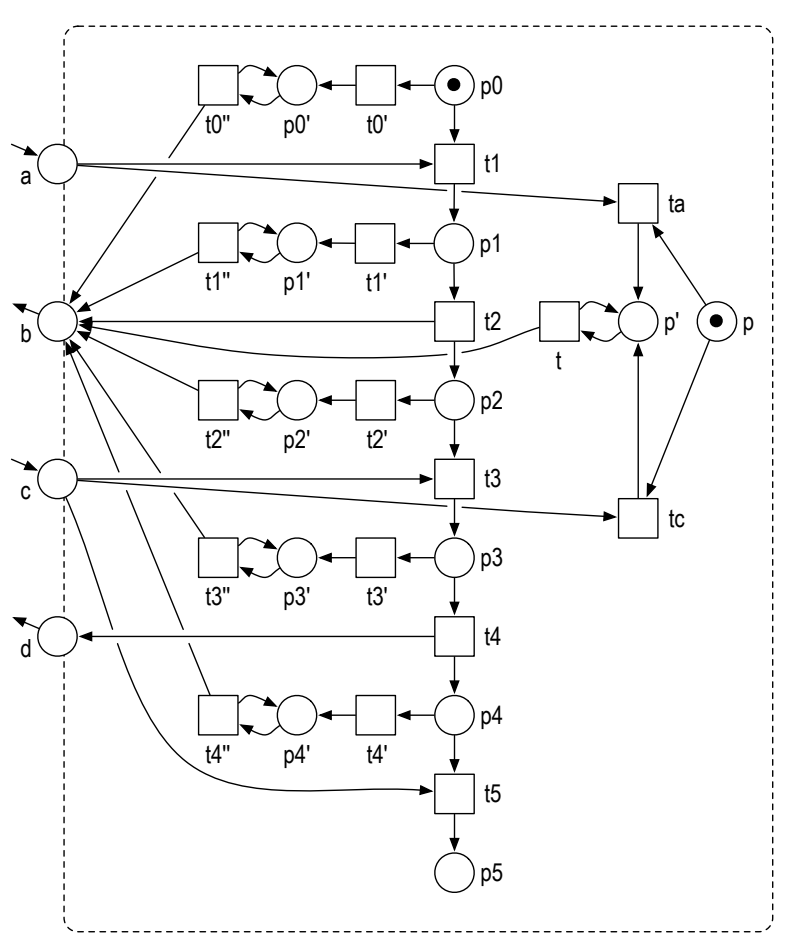

(b) $N_{w, b}$

Fig. 5: Construction of $N_{w}$ and $N_{w, b}$ for $w=a b c d c$ with $b, d \in I$ and $a, c \in O$

removed the token from $p$. These facts imply that the Parikh vectors of $w$ and $v$ agree: Each $t_{i}$ consumes a token from or produces a token on $w_{i}$, but all interface places are empty at the end of the traces.

In $u$, each occurrence of $t_{j}$ with $w_{j} \in t_{j}^{\bullet}$ (as output place of $N_{w, o}$, i.e., $w_{j} \in I$ ) is paired with a succeeding occurrence of $w_{j}$ (as transition of $e n v\left(N_{w, o}\right)$ ); otherwise, transition $w_{j}$ would be enabled at $m_{2}$ in $\operatorname{env}\left(N_{w, o}\right)$ and $m_{2}$ would not be a stop except for inputs. As transition $w_{j}$ is not in conflict with any other transition of $\operatorname{env}\left(N_{w, o}\right)$, we assume that $w_{j}$ fires immediately after $t_{j}$. In the corresponding rearranged trace $v^{\prime}$ of $v$, all $w_{j} \in I$ occur in the same order as in $w$, and $v^{\prime}$ still leads to $m_{2}$.

Similarly, each occurrence of $t_{j}$ with $w_{j} \in \bullet^{\bullet}$ (as input place of $N_{w, o}$, i.e., $\left.w_{j} \in O\right)$ is paired with a preceding occurrence of $w_{j}$ (as transition of $e n v\left(N_{w, o}\right)$ ), which can be delayed such that it occurs immediately before $t_{j}$. In the corresponding rearranged trace $v^{\prime \prime}$ of $v^{\prime}$, all $w_{j} \in O$ occur in the same order as in $w$, because $v^{\prime}$ and $v^{\prime \prime}$ have the same Parikh vector as $w$; thus, $v^{\prime \prime}$ is $w$.

We have transformed $v$ to $w$ by moving $w_{j} \in I$ backwards and $w_{j} \in O$ forwards. This can also be done in the run underlying $v$ in env(Spec), because 
the respective transitions have an empty preset and postset, respectively. Thus,

$$
m_{\text {env }(\text { Spec })} \stackrel{w}{\Longrightarrow} m_{1}\left(\text { and } m_{\text {env }\left(N_{w, o}\right)} \stackrel{w}{\Longrightarrow} m_{2}\right)
$$

and therefore $w \in \operatorname{stop}($ Spec $)$.

Example 3.4. For the open net $S^{\prime}$ in Fig. 3, we have $\operatorname{stop}\left(S^{\prime}\right)=\left\{\left.w \in\{r, t\}^{*}|| w\right|_{t}=|w|_{r}+1 \wedge \forall v \sqsubseteq w:|v|_{t} \leq|v|_{r}+1\right\} \subseteq \operatorname{stop}(S)$ (see Example 3.2). We conclude with Theorem 3.8 that $S^{\prime} r$-accords with $S$.

Accordance, as defined in Definition 3.3 does not guarantee compositionality; that is, it is not a precongruence w.r.t. open net composition $\oplus$. We showed this in Example 3.1 using open nets $S$ and $S^{\prime}$. To see the difference between $S$ and $S^{\prime}$ consider any trace of env $(S)$ not containing $e$. In the marking reached, there is a token on $p_{0}, p_{1}$, or $e^{o}$ and the trace $r e$ is always possible. In contrast, env $\left(S^{\prime}\right)$ can always refuse re, because env $\left(S^{\prime}\right)$ can never perform an $e$. Therefore, it is not possible to differentiate between $S$ and $S^{\prime}$ with something even weaker than standard failure semantics, as introduced by [7] (like the trace semantics we employed in Definition 3.4.

\subsection{Deriving the coarsest precongruence for responsiveness}

Taking into account the example in Fig. 4 and the observation that refusal information is necessary to distinguish open nets in terms of $r$-accordance, we shall characterize the precongruence $\sqsubseteq_{r, a c c}^{c}$ in terms of a variant of failure semantics. For this, we will not use CSP failures, as introduced by Brookes et al. [7, but Vogler's $\mathcal{F}^{+}$-semantics [33. Whereas a failure is a pair $(w, X)$ where $w$ is a trace of a net and $X$ is a subset of the alphabet - a refusal set - the $\mathcal{F}^{+}$-semantics is a stronger notion, considering pairs $(w, X)$ where $X$ is a set of traces; such a pair is a tree failure.

Definition $3.9\left(\mathcal{F}^{+}\right.$-semantics). The $\mathcal{F}^{+}$-semantics of a labeled net $N$ is

$$
\begin{aligned}
& \mathcal{F}^{+}(N)=\left\{(w, X) \in \Sigma^{*} \times \mathcal{P}\left(\Sigma^{+}\right) \mid\right. \exists m \in M_{N}: \\
&\left.m_{N} \stackrel{w}{\Longrightarrow} m \wedge \nexists w^{\prime} \in X: m \stackrel{w^{\prime}}{\Longrightarrow}\right\} .
\end{aligned}
$$

We say that after executing $w, N$ refuses $X$.

Example 3.5. We can distinguish the open nets $S$ and $S^{\prime}$ in Fig. 1a and Fig. 3 by their $\mathcal{F}^{+}$-semantics: We have $\left(\varepsilon, r^{*} e\right) \in \mathcal{F}^{+}\left(S^{\prime}\right)$ (because no trace of $S^{\prime}$ contains an $e$ ) but $\left(\varepsilon, r^{*} e\right) \notin \mathcal{F}^{+}(S)$ (because we cannot prevent trace re after trace $\varepsilon$, as explained below Example 3.4.

If we consider the composition $N_{1} \oplus N_{2}$ of two open nets $N_{1}$ and $N_{2}$, then its $\mathcal{F}^{+}$-semantics coincides with that of the parallel composition of the two environments, env $\left(N_{1}\right) \Uparrow \operatorname{env}\left(N_{2}\right)$. 
Lemma 3.10. For two composable open nets $N_{1}$ and $N_{2}$, we have

$$
\mathcal{F}^{+}\left(\operatorname{env}\left(N_{1} \oplus N_{2}\right)\right)=\mathcal{F}^{+}\left(\operatorname{env}\left(N_{1}\right) \Uparrow \operatorname{env}\left(N_{2}\right)\right) .
$$

Proof. Follows directly from Lemma 2.12 If one net has a tree failure $(w, X)$ due to a marking $m$, then the other net can reach an agreeing marking $m^{\prime}$ via the trace $w$. If some trace $v \in X$ could be performed from $m^{\prime}$, this would also be possible from $m$ due to weak bisimilarity, yielding a contradiction. Thus, $(w, X)$ is also a tree failure of the other net.

Before determining the $\mathcal{F}^{+}$-semantics for the composition of two open nets, we first recall how this can be done for the composition of two labeled nets 33 , Theorem 3.3.15] and for the hiding of common actions [33, Theorem 3.4.2]; we recall this to prepare the next section, where we study a new variation of the $\mathcal{F}^{+}$-semantics.

Proposition 3.11 $\left(\mathcal{F}^{+}\right.$-semantics for labeled net composition [33]). For two composable labeled nets $N_{1}$ and $N_{2}$, we have

$$
\begin{aligned}
\mathcal{F}^{+}\left(N_{1} \| N_{2}\right)=\{(w, X) \mid & \exists\left(w_{1}, X_{1}\right) \in \mathcal{F}^{+}\left(N_{1}\right),\left(w_{2}, X_{2}\right) \in \mathcal{F}^{+}\left(N_{2}\right): \\
w & \in w_{1} \| w_{2} \wedge \forall x \in X: \\
& \left.x \in x_{1} \| x_{2} \text { implies } x_{1} \in X_{1} \vee x_{2} \in X_{2}\right\} .
\end{aligned}
$$

In the next proposition recapitulates [33, Theorem 3.4.2]. We consider a labeled net $N / A, A \subseteq \Sigma$ and use $\phi(w)$ to denote $\left.w\right|_{\Sigma \backslash A}$. We canonically extend the notion of $\phi(w)$ pointwise to sets of traces.

Proposition 3.12 ([33]). For any labeled net $N$ and any label set $A \subseteq \Sigma_{N}^{*}$, we have

$$
\mathcal{F}^{+}(N / A)=\left\{(\phi(w), X) \mid\left(w, \phi^{-1}(X)\right) \in \mathcal{F}^{+}(N)\right\} .
$$

We now combine Lemma 3.10 and Propositions 3.12 and 3.11 to show how the $\mathcal{F}^{+}$-semantics for the composition of two open nets can be determined.

Proposition $3.13\left(\mathcal{F}^{+}\right.$-semantics for open net composition). For two composable open nets $N_{1}$ and $N_{2}$, we have

$$
\begin{aligned}
\mathcal{F}^{+}\left(N_{1} \oplus N_{2}\right)=\{(w, X) \mid & \exists\left(w_{1}, X_{1}\right) \in \mathcal{F}^{+}\left(N_{1}\right),\left(w_{2}, X_{2}\right) \in \mathcal{F}^{+}\left(N_{2}\right): \\
w & \in w_{1} \Uparrow w_{2} \wedge \forall x \in X: \\
& \left.x \in x_{1} \Uparrow x_{2} \text { implies } x_{1} \in X_{1} \vee x_{2} \in X_{2}\right\} .
\end{aligned}
$$

Proof. Proposition 3.11 shows that the right part of this equation - with $\|$ replacing $\Uparrow$-is equal to $\mathcal{F}^{+}\left(e n v\left(N_{1}\right) \| e n v\left(N_{2}\right)\right)$; then, one can hide the common actions of env $\left(N_{1}\right)$ and env $\left(N_{2}\right)$, and by Proposition 3.12 the right hand side is equal to $\mathcal{F}^{+}\left(\operatorname{env}\left(N_{1}\right) \Uparrow \operatorname{env}\left(N_{2}\right)\right)$; the latter is equal to $\mathcal{F}^{+}\left(\operatorname{env}\left(N_{1} \oplus N_{2}\right)\right)=\mathcal{F}^{+}\left(N_{1} \oplus N_{2}\right)$ by Lemma 3.10 .

For the present setting, the tree failures used in the $\mathcal{F}^{+}$-semantics give too much information about the moment of choice in an open net. This information can be removed by closing up under an ordering over tree failures. The resulting modification of the $\mathcal{F}^{+}$-semantics yields the following refinement relation. 
Definition 3.14 $\left(\mathcal{F}^{+}\right.$-refinement). For two interface-equivalent labeled nets Impl and Spec, Impl $\mathcal{F}^{+}$-refines Spec, denoted by Impl $\sqsubseteq_{\mathcal{F}+}$ Spec, if

$$
\forall(w, X) \in \mathcal{F}^{+}(\operatorname{Impl}): \exists x \in\{\varepsilon\} \cup \downarrow X:\left(w x, x^{-1} X\right) \in \mathcal{F}^{+}(\text {Spec }) .
$$

For two interface-equivalent open nets $I m p l$ and $S p e c$, we define $I m p l \sqsubseteq_{\mathcal{F}_{f i n}^{+}} S p e c$, if $e n v(\operatorname{Impl}) \sqsubseteq_{\mathcal{F}_{\text {fin }}^{+}} \operatorname{env}(\mathrm{Spec})$.

Example 3.6. We have $\left(\varepsilon, r^{*} e\right) \in \mathcal{F}^{+}\left(S^{\prime}\right)$ (see Example 3.5) but for all $x \in$ $\{\varepsilon\} \cup \downarrow r^{*} e,\left(x, x^{-1} r^{*} e\right) \notin \mathcal{F}^{+}(S)$ because $r^{-1} r^{*} e=r^{*} e$. Thus, $S^{\prime}$ does not $\mathcal{F}^{+}$-refine $S$.

Using $\mathcal{F}^{+}$-semantics and -refinement is a technically very beneficial reformulation 29] of $\mathcal{F}^{++}$-inclusion in 33 . Our definition of $\mathcal{F}^{+}$-refinement is equivalent to the definition of the refinement relation $\sqsubseteq_{\mathcal{F}+}$ in 29 , which coincides with should (or fair) testing [6 29] as proved in [29, Theorem 36]. Should testing is a precongruence for composition [29, and with the help of Lemma 3.10, we can show that it is also a precongruence for the composition operator $\oplus$.

Theorem 3.15 (precongruence). $\mathcal{F}^{+}$-refinement is a precongruence for the composition operator $\oplus$.

Proof. Let Impl and Spec be interface-equivalent open nets with $I m p l \sqsubseteq{ }_{\mathcal{F}}+$ Spec, and let $C$ be an open net composable with both. We have to show, $\operatorname{Impl} \oplus C \sqsubseteq_{\mathcal{F}+}$ Spec $\oplus C$.

We have $\mathcal{F}^{+}(\operatorname{env}($ Spec $\oplus C))=\mathcal{F}^{+}(\operatorname{env}($ Spec $) \Uparrow \operatorname{env}(C))$ by Lemma 3.10 . Let $A$ denote the common actions of env(Spec) and env $(C)$. Then we can replace operator $\Uparrow$ with operator $\|$ and make the hiding explicit, which results in $\mathcal{F}^{+}($env $($Spec $) \Uparrow e n v(C))=\mathcal{F}^{+}(($env $($Spec $) \| \operatorname{env}(C)) / A)$. Likewise, we derive $\mathcal{F}^{+}(\operatorname{env}(\operatorname{Impl} \oplus C))=\mathcal{F}^{+}((\operatorname{env}(\operatorname{Impl}) \| \operatorname{env}(C)) / A)$.

Impl $\mathcal{F}^{+}$-refines Spec and in [29, Lemma 46] $\mathcal{F}^{+}$-refinement for labeled transition systems has been proved to be a precongruence for the composition operator $\|$, and it is also preserved under hiding of common actions [29, Lemma 45]. Thus, we obtain that $(e n v(\operatorname{Impl}) \| \operatorname{env}(C)) / A \sqsubseteq_{\mathcal{F}^{+}}(\operatorname{env}(\operatorname{Spec}) \| \operatorname{env}(C)) / A$. As this only depends on the $\mathcal{F}^{+}$-semantics of the two nets, the equations above show $\operatorname{Impl} \oplus C \sqsubseteq_{\mathcal{F}+} \operatorname{Spec} \oplus C$.

The proof of Theorem 3.15 gives the following result.

Proposition 3.16. $\mathcal{F}^{+}$-refinement is a precongruence for $\|$and hiding on labeled nets.

With the next theorem, we prove that $\mathcal{F}^{+}$-refinement is the coarsest precongruence that is contained in the $r$-accordance relation.

Theorem 3.17 (precongruence and $\mathcal{F}^{+}$-refinement coincide). For two interface-equivalent open nets Impl and Spec, we have

$$
\operatorname{Impl} \sqsubseteq_{\mathcal{F}+} \text { Spec iff Impl } \sqsubseteq_{r, a c c}^{c} \text { Spec } .
$$


Proof. $\Rightarrow:$ In the following, we assume a trace $w \in \operatorname{stop}(\operatorname{Impl})$ and prove $w \in$ stop (Spec). Then, applying Theorem 3.8, we get Impl $\sqsubseteq_{r, a c c}$ Spec, which in turn shows the claim with Theorem 3.15 and the definition of $\complement_{r, a c c}^{c}$.

So let $O$ be the set of output places of $I m p l$ and, equivalently, of Spec. We have $w \in \operatorname{stop}($ Impl $)$ if and only if $(w, O) \in \mathcal{F}^{+}($Impl) by Definition 3.4 and 3.9. Then, by Impl $\sqsubseteq_{\mathcal{F}+}$ Spec, there must be a suitable $x \in\{\varepsilon\} \cup \downarrow O=\{\varepsilon\} \cup O$ that makes the defining condition of Definition 3.14 true. We cannot have $x \in O$ because $(w x,\{\varepsilon\}) \notin \mathcal{F}^{+}($Spec $)$by Definition 3.9. Thus, $x=\varepsilon$ and $(w, O) \in \mathcal{F}^{+}($Spec $)$, implying $w \in \operatorname{stop}($ Spec $)$.

$\Leftarrow$ : Let $(w, X) \in \mathcal{F}^{+}(\operatorname{Impl})$. In addition, consider an open net $C$ with the new output $x$ and the new input $y$. Open net $C$ has the empty initial marking, no final marking, and contains only a single transition that can indefinitely repeat to produce a token in $x$ while consuming a token from $y$. The idea is to construct an open net $N$ from $(w, X)$ such that $C$ is not an $r$-controller of Impl $\oplus N$ because of $(w, X)$. For the moment, assume such a net $N$ has been constructed. By Impl $\sqsubseteq_{r, a c c}^{c}$ Spec and because $\sqsubseteq_{r, a c c}^{c}$ is a precongruence, we have $\operatorname{Impl} \oplus N \sqsubseteq_{r, a c c}^{c} \operatorname{Spec} \oplus N$ and thus $\operatorname{Impl} \oplus N \sqsubseteq_{r, a c c}$ Spec $\oplus N$ by Definition 3.3 . Thus, $C$ is also not an $r$-controller of Spec $\oplus N$, and from this we will conclude that $(w, X)$ is covered by $\mathcal{F}^{+}$(Spec) according to Definition 3.14. Then we will have proved $I m p l \sqsubseteq_{\mathcal{F}^{+}}$Spec.

As to the actual construction of $N$ : The open net $N$ has inputs $I=O_{\operatorname{Impl}} \uplus$ $\{x\}$ and outputs $O=I_{\text {Impl }} \uplus\{y\}$ and enables a transition sequence $v=t_{1} \ldots t_{k}$. Each transition in $v$ is connected to an interface place of $N$ such that the corresponding trace of interface actions is $w$; that is, $N$ contains net $N_{w}$ as in Fig. 5 (except that $p_{k}$ is called $p_{\varepsilon}$ here). Thus, we can essentially fire the trace $w$ of $e n v(N)$ in $\operatorname{Impl} \oplus N$ and, therefore, in $\operatorname{Impl} \oplus N \oplus C$ by firing $v$ instead of the labeled transitions. This way, we reach in $\operatorname{Impl}$ the marking $m$ that refuses $X$ in $e n v(\operatorname{Impl})$; in $N$, there is only one token in the place $p_{\varepsilon}$ and the token in the place $p$ has been consumed. This token is necessary to enable a transition $t^{\prime}$ that is essential for responsiveness; that is, transitions $t$ and $t^{\prime}$ would repeatedly communicate with $C$. The place $p$ can only be marked again by firing some transition $t_{z}^{\prime}$ with $z \in X$, and this in turn requires the firing of a transition sequence that - similarly to $v$-looks to $\operatorname{Impl}$ (or rather env(Impl)) like trace $z$. But this trace cannot be fired at $m$; hence, $C$ is not an $r$-controller of $\operatorname{Impl} \oplus N$.

To achieve the effect just described, the second part of the open net $N$ encodes the tree part $X$ of tree failure $(w, X)$. Common prefixes thereby correspond to the same path in the $X$-part of $N$. When reaching an element of $X$, a token can be produced in the place $p$. Figure 6 illustrates this construction; it is a small adaptation of a construction that is used in [33, Fig. 3.19].

Let $w=w_{1} \ldots w_{k}$ such that for $j=1, \ldots, k, w_{j} \in I_{\text {Impl }} \uplus O_{\text {Impl }}$. Define the open net $N=\left(P, T, F, m_{N}, O, I, \emptyset\right)$ by

$$
\begin{aligned}
-P= & \{p\} \\
& \uplus\left\{p_{i} \mid 0 \leq i \leq k-1\right\} \\
& \uplus\left\{p_{u} \mid u \in \downarrow X \cup\{\varepsilon\}\right\}
\end{aligned}
$$




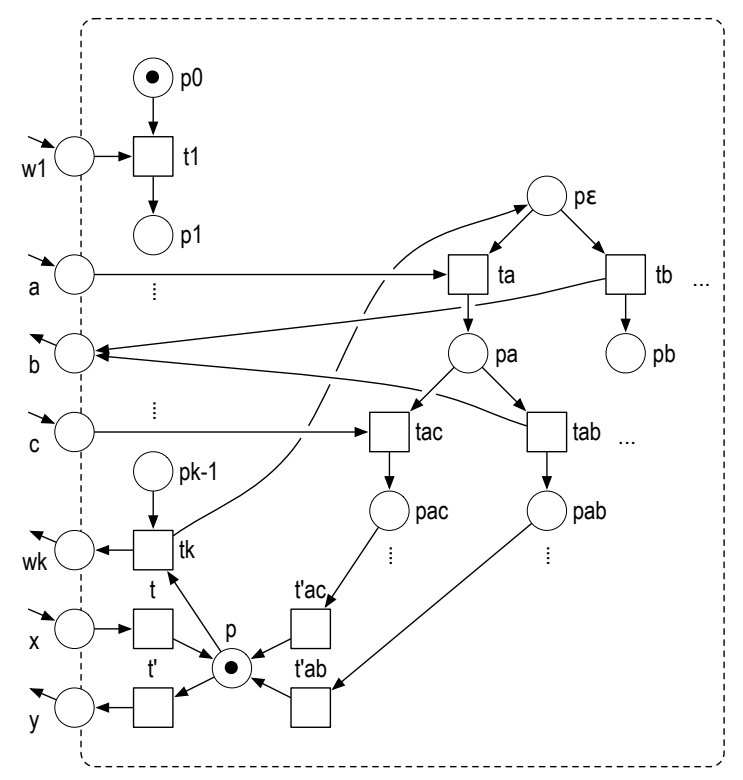

Fig. 6: Illustration of the construction of open net $N$.

$$
\begin{aligned}
-T= & \left\{t, t^{\prime}\right\} \\
& \uplus\left\{t_{i} \mid 1 \leq i \leq k\right\} \\
& \uplus\left\{t_{u} \mid u \in \downarrow X \wedge u \neq \varepsilon\right\} \\
& \uplus\left\{t_{z}^{\prime} \mid z \in X\right\} \\
-F= & \left\{\left(p_{i}, t_{i+1}\right) \mid 0 \leq i \leq k-1\right\} \\
& \uplus\left\{\left(t_{i}, p_{i}\right) \mid 1 \leq i \leq k-1\right\} \\
& \uplus\left\{(x, t),(t, p),\left(p, t^{\prime}\right),\left(t^{\prime}, y\right),\left(p, t_{k}\right),\left(t_{k}, p_{\varepsilon}\right)\right\} \\
& \uplus\left\{\left(p_{u}, t_{u a}\right) \mid a \in I_{\text {Impl }} \uplus O_{\text {Impl }} \wedge u a \in \downarrow X\right\} \\
& \uplus\left\{\left(t_{u}, p_{u}\right) \mid u \in \downarrow X \wedge u \neq \varepsilon\right\} \\
& \uplus\left\{\left(p_{z}, t_{z}^{\prime}\right),\left(t_{z}^{\prime}, p\right) \mid z \in X\right\} \\
& \uplus\left\{\left(w_{i}, t_{i}\right) \mid 1 \leq i \leq k \wedge w_{i} \in O_{\text {Impl }}\right\} \\
& \uplus\left\{\left(t_{i}, w_{i}\right) \mid 1 \leq i \leq k \wedge w_{i} \in I_{\text {Impl }}\right\} \\
& \uplus\left\{\left(a, t_{u a}\right) \mid a \in O_{\text {Impl }} \wedge u a \in \downarrow X\right\} \\
& \uplus\left\{\left(t_{u a}, a\right) \mid a \in I_{\text {Impl }} \wedge u a \in \downarrow X\right\}, \text { and } \\
-m_{N}= & {\left[p_{0}, p\right] . }
\end{aligned}
$$

As argued previously, we now have that $C$ is not an $r$-controller of $\operatorname{Spec} \oplus N$; that is, a marking $m_{1}$ can be reached in Spec $\oplus N \oplus C$ where responsiveness is violated. Clearly, $p$ must be empty in $m_{1}$; thus, $v$ has been fired in $N$ and possibly also some transitions in its $X$-part. There is only one token in the places of inner $(N)$, and it is in some $p_{u}$ with $u \in \downarrow X \cup\{\varepsilon\}$. Let $m_{2}$ be the projection of $m_{1}$ onto the places of Spec. From the point of view of Spec, we have fired a trace $w u$ of env(Spec), reaching $m_{2}$. Because in Spec $\oplus N \oplus C$ no $t_{u u^{\prime}}^{\prime}$ can become enabled (otherwise, $C$ would be an $r$-controller), $u^{\prime}$ cannot be 
fired in env(Spec) at $m_{2}$. Thus, $\left(w u,\left\{u^{\prime} \mid u u^{\prime} \in X\right\}\right) \in \mathcal{F}^{+}($Spec $)$and, therefore, Impl $\mathcal{F}^{+}$Spec.

Example 3.7. We already showed with Fig. 4 that for the open nets $S$ and $S^{\prime}$ $S^{\prime} \sqsubseteq_{r, a c c}^{c} S$ does not hold. This is now confirmed with Theorem 3.17 because $S^{\prime}$ does not $\mathcal{F}^{+}$-refine $S$ by Example 3.6 .

\section{Unbounded nets and final markings}

In this section, we consider possibly unbounded open nets and final markings. We refer to the resulting variant of responsiveness as final-responsiveness or $f$-responsiveness for short.

Definition 4.1 ( $f$-responsiveness). Let $N_{1}$ and $N_{2}$ be composable open nets. A marking $m$ of $N_{1} \oplus N_{2}$ is $f$-responsive if either $m$ is responsive or we can reach a final marking of $N_{1} \oplus N_{2}$ from $m$. Open nets $N_{1}$ and $N_{2}$ are $f$-responsive if their composition $N_{1} \oplus N_{2}$ is a closed net and every reachable marking in $N_{1} \oplus N_{2}$ is $f$-responsive.

The notion of $f$-responsiveness generalizes responsiveness defined in Definition 3.1. While responsiveness requires at least one net of the composition to repeatedly talk to the other net, $f$-responsiveness also allows the composition to terminate instead - that is, to reach a common final marking.

Next, we redefine the notion of a controller and accordance for this variant of responsiveness which yields $f r$-controllers and $f r$-accordance. Again, because $f r$-accordance will turn out not to be a precongruence, we also introduce its coarsest precongruence.

As for responsiveness, for every open net $N$, there exists an $f r$-controlleragain, this open net just has to continuously send a message.

Definition 4.2 ( $f r$-controller, $f r$-accordance). An open net $C$ is an $f r$-controller of an open net $N$ if $N$ and $C$ are $f$-responsive.

For two interface-equivalent open nets Impl and Spec, Impl fr-accords with Spec, denoted by Impl $\sqsubseteq_{f r, a c c} S p e c$, if for all open nets $C: C$ is an $f r$-controller of Spec implies $C$ is an $f r$-controller of Impl.

We denote the coarsest precongruence w.r.t. $\oplus$ contained in $\sqsubseteq_{f r, a c c}$ by $\sqsubseteq_{f r, a c c}^{c}$.

Example 4.1. The open net $C^{\prime}$ in Fig. 7a represents another client for the unreliable time server $S$ in Fig. 1a. The client $C^{\prime}$ repeatedly updates its system time and responds with a response packet. However, if the time server sends an error message, $C^{\prime}$ receives this message (input place $e$ ) and terminates (final marking $\left[p_{4}\right]$ ). The open nets $S$ and $C^{\prime}$ are composable; their composition $S \oplus C^{\prime}$ is a closed net, which is depicted in Fig. $7 \mathrm{~b} C^{\prime}$ is not an $r$-controller of $S$, because the nonresponsive marking $\left[p_{4}\right]$ is reachable in their composition $S \oplus C^{\prime}$. In contrast, $C^{\prime}$ is an $f r$-controller of $S$ because $\left[p_{4}\right]$ is a final marking of $S \oplus C^{\prime}$. 


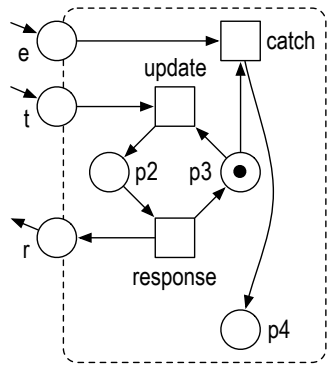

(a) Open net $C^{\prime}$

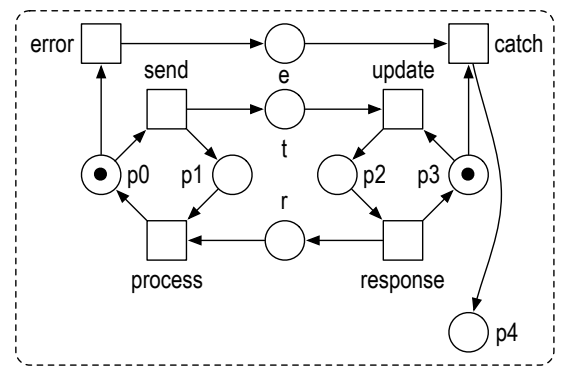

(b) Open net $S \oplus C^{\prime}$

Fig. 7: Open net $C^{\prime}$ modeling a terminating client for the open net $S$ in Fig. 1a and their composition $S \oplus C^{\prime}$. In addition to the models, we have $\Omega_{C^{\prime}}=\Omega_{S \oplus C^{\prime}}=$ $\left\{\left[p_{4}\right]\right\}$.

The open net $S^{\prime}$ in Fig. 3 fr-accords with the open net $S$ in Fig. 1a Every fr-controller $C$ of $S$ must provide a token on $r$ for each token on $t$; otherwise, $S$ can get stuck in a nonfinal marking with a token on $p_{1}$. (Additionally, the $f r$ controller must be able to consume a token from $e$ and reach a final marking.) Thus, $S^{\prime}$ and $C$ are responsive and $C$ is an $f r$-controller of $S^{\prime}$.

Like $r$-accordance, also $f r$-accordance does not guarantee compositionality; that is, it is not a precongruence with respect to open net composition $\oplus$. Consider again Fig. 4 for a counterexample. The open net $S^{\prime} f r$-accords with the open net $S$, but $S^{\prime} \oplus A$ does not $f r$-accord with $S \oplus A$, because the open net $B$ is an $f r$-controller of $S \oplus A$ but not of $S^{\prime} \oplus A$ : The only final marking $\left[p_{5}, p_{6}\right]$ is not reachable in $\left(S^{\prime} \oplus A\right) \oplus B$, and the trace $x$ is a stop-trace of both $S^{\prime} \oplus A$ and $B$.

We continue by first giving a trace-based characterization for $f r$-accordance. Afterward, we characterize the coarsest precongruence that is contained in the fr-accordance relation.

\subsection{A trace-based semantics for final-responsiveness}

We extend Definition 3.4 by a set of dead-traces, a weak version of a notion with the same name in 3132. A dead-trace is a stop-trace leading to a nonfinal stop except for inputs - that is, a marking that is dead except for inputs.

Definition 4.3 (stopdead-semantics). Let $N$ be a labeled net. A marking $m$ of $N$ is dead except for inputs if $m$ is a stop except for inputs and there exists no final marking $m^{\prime}$ of $N$ with $m \stackrel{\varepsilon}{\Longrightarrow} m^{\prime}$. The stopdead-semantics of $N$ is defined by the sets of traces

$-\operatorname{stop}(N)$ and

$-\operatorname{dead}(N)=\left\{w \mid m_{N} \stackrel{w}{\Longrightarrow} m\right.$ and $m$ is dead except for inputs $\}$. 
Example 4.2. For the open nets $S$ and $C^{\prime}$ in Fig. 1a and 7a, [] is the only final marking of $S$ and $\left[p_{4}\right]$ is the only final marking $C^{\prime}$. Therefore, we have

$\operatorname{dead}(S)=\left\{\left.w \in\{r, t\}^{*}|| w\right|_{t}=|w|_{r}+1 \wedge \forall v \sqsubseteq w:|v|_{t} \leq|v|_{r}+1\right\}$ $\cup\left\{\left.w e v \in L(S)|| w v\right|_{t}<|w v|_{r}\right\}$

Furthermore, we have

$\operatorname{stop}\left(C^{\prime}\right)=\operatorname{stop}(C) \cup\left\{\left.w e v\left|w \in\{r, t\}^{*} \wedge v \in\{r, t, e\}^{*} \wedge \forall u \sqsubseteq w v:\right| u\right|_{r} \leq|u|_{t}\right\}$

and

$\operatorname{dead}\left(C^{\prime}\right)=\operatorname{stop}(C) \cup\left\{w e v \mid w \in\{r, t\}^{*} \wedge v \in\{r, t, e\}^{*} \wedge \forall u \sqsubseteq w v:\right.$

$\left.|u|_{r} \leq|u|_{t} \wedge\left(|w v|_{t}>|w v|_{r} \vee|v|_{e} \geq 1\right)\right\}$

where $\operatorname{stop}(C)$ has been defined in Example 3.2 .

The presence of dead-traces in open nets $N_{1}$ and $N_{2}$ is closely related to the question whether $N_{1}$ and $N_{2}$ are $f$-responsive. We continue by relating first $f$-responsive and then the stopdead-semantics to markings that are dead except for inputs.

Lemma 4.4 ( $f$-responsive vs. dead except for inputs). Let $N_{1}$ and $N_{2}$ be composable open nets such that $N_{1} \oplus N_{2}$ is a closed net. Let $E=\operatorname{env}\left(N_{1}\right) \| \operatorname{env}\left(N_{2}\right)$, and let $m$ be a marking of $N_{1} \oplus N_{2}$ and $\bar{m}$ be a marking of $E$ such that $m$ and $\bar{m}$ agree. If $m$ is $f$-responsive, then $\bar{m}$ is not dead except for inputs. If $m$ and $\bar{m}$ strongly agree, the converse also holds.

Proof. We have $N_{1} \oplus N_{2}=e n v\left(N_{1} \oplus N_{2}\right)=: C$ and $\left(I_{1} \cap O_{2}\right) \uplus\left(I_{2} \cap O_{1}\right)=$ $O_{1} \uplus O_{2}=: O$, because $N_{1} \oplus N_{2}$ is a closed net. Note that only transitions in $O$ are not $\tau$-labeled in $E$.

$\Rightarrow$ : If $m$ is responsive or if $m$ and $\bar{m}$ do not strongly agree, we are done by Lemma 3.5. Otherwise, there is a final marking $m^{\prime}$ of $C$ reachable from $m$. According to Proposition 2.11(1), there is a marking $\bar{m}^{\prime}$ reachable from $\bar{m}$ in $E$ such that $m^{\prime}$ and $\bar{m}^{\prime}$ agree (even strongly). Marking $\bar{m}^{\prime}$ is a final marking of $E$ by Proposition 2.11 (3). Thus, $\bar{m}$ is not dead except for inputs by Definition 4.3 .

$\Leftarrow$ : If $\bar{m}$ is not a stop except for inputs, then $m$ is responsive by Lemma 3.5 and therefore $f$-responsive. Otherwise, there is a final marking $\bar{m}^{\prime}$ of $E$ reachable from $\bar{m}$. Applying Proposition 2.11(2), there is a marking $m^{\prime}$ reachable from $m$ in $C$ such that $m^{\prime}$ and $\bar{m}^{\prime}$ agree. Marking $m^{\prime}$ is a final marking of $C$ by Proposition 2.11(3), proving $f$-responsiveness of $m$.

Lemma 4.5 (dead except for inputs vs. stopdead-semantics). Let $N_{1}$ and $N_{2}$ be composable open nets, and let $E=\operatorname{env}\left(N_{1}\right) \| \operatorname{env}\left(N_{2}\right)$. Let $m_{1}$ and $m_{2}$ be markings of env $\left(N_{1}\right)$ and env $\left(N_{2}\right)$, respectively. Then, $m=m_{1}+m_{2}$ is dead except for inputs in $E$ iff $m_{1}$ is a stop except for inputs and $m_{2}$ is dead except for inputs, or vice versa.

Proof. $\Rightarrow$ : Because $m$ is a stop except for inputs by Definition 4.3 , both $m_{1}$ and $m_{2}$ are stops except for inputs by Lemma 3.6. Assume neither $m_{1}$ nor $m_{2}$ are dead except for inputs due to $m_{1}^{\prime}$ and $m_{2}^{\prime}$ respectively. Then $m=m_{1}+m_{2} \stackrel{\varepsilon}{\Longrightarrow}$ $m_{1}^{\prime}+m_{2}^{\prime}$ by Proposition 2.8 and $m_{1}^{\prime}+m_{2}^{\prime}$ is a final marking by Proposition 2.11(3). This contradicts the assumption. 
$\Leftarrow$ : Due to Lemma 3.6, $m$ is a stop except for inputs. W.l.o.g., assume $m_{2}$ is dead except for inputs. Whenever $m \stackrel{\varepsilon}{\Longrightarrow} m^{\prime}$, Proposition 2.8 gives us $m_{1} \stackrel{\varepsilon}{\Longrightarrow} m_{1}^{\prime}$ and $m_{2} \stackrel{\varepsilon}{\Longrightarrow} m_{2}^{\prime}$ where neither $m_{2}^{\prime}$ nor-by Proposition 2.11(3)- $m^{\prime}=m_{1}^{\prime}+m_{2}^{\prime}$ are final. Thus, $m$ is dead except for inputs in $E$ by Definition 4.3

We combine Lemma 4.4 and Lemma 4.5 and show how the stop dead-semantics can be used to characterize $f$-responsiveness.

Proposition 4.6 ( $f$-responsiveness vs. stopdead-semantics). Let $N_{1}$ and $N_{2}$ be composable open nets such that $N_{1} \oplus N_{2}$ is a closed net. Then

$$
\begin{aligned}
& N_{1} \text { and } N_{2} \text { are } f \text {-responsive iff } \operatorname{stop}\left(N_{1}\right) \cap \operatorname{dead}\left(N_{2}\right)=\emptyset \text { and } \\
& \operatorname{dead}\left(N_{1}\right) \cap \operatorname{stop}\left(N_{2}\right)=\emptyset \text {. }
\end{aligned}
$$

Proof. Let $C=N_{1} \oplus N_{2}$ and $E=e n v\left(N_{1}\right) \| e n v\left(N_{2}\right)$.

$\Rightarrow$ : Proof by contraposition. W.l.o.g., we assume a trace $w \in \operatorname{stop}\left(N_{1}\right) \cap$ $\operatorname{dead}\left(N_{2}\right)$. Hence, $m_{\text {env }\left(N_{1}\right)} \stackrel{w}{\Longrightarrow} m_{1}$ in $\operatorname{env}\left(N_{1}\right)$ and $m_{\text {env }\left(N_{2}\right)} \stackrel{w}{\Longrightarrow} m_{2}$ in $\operatorname{env}\left(N_{2}\right)$ such that $m_{1}$ is a stop except for inputs and $m_{2}$ is dead except for inputs. By Lemma 4.5 $m_{1}+m_{2}$ is dead except for inputs in E. By Proposition 2.11(2), a marking $m$ is reachable in $C$ such that $m$ and $m_{1}+m_{2}$ agree, and $m$ is not $f$-responsive by Lemma 4.4 .

$\Leftarrow$ : Proof by contraposition. Assume $m_{C} \stackrel{\varepsilon}{\Longrightarrow} m$ in $C$ such that $m$ is not $f$-responsive. Applying Proposition 2.11(1), we can reach some $m_{1}+m_{2}$ in $E$ (with $m_{i}$ a marking of $N_{i}, i=1,2$ ) such that $m$ and $m_{1}+m_{2}$ strongly agree, and, by Lemma 4.4, $m_{1}+m_{2}$ is dead except for inputs. By Proposition 2.8, we

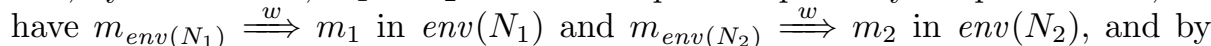
Lemma 4.5. $m_{1}$ is a stop except for inputs and $m_{2}$ is dead except for inputs, or vice versa. Thus, $w \in \operatorname{stop}\left(N_{1}\right) \cap \operatorname{dead}\left(N_{2}\right)$ or $w \in \operatorname{dead}\left(N_{1}\right) \cap \operatorname{stop}\left(N_{2}\right)$.

Example 4.3. Consider again Examples 3.2 and 4.2 One can see that stop $(S) \cap$ $\operatorname{dead}\left(C^{\prime}\right)=\emptyset$ and $\operatorname{dead}(S) \cap \operatorname{stop}\left(C^{\prime}\right)=\emptyset$; thus, $C^{\prime}$ is indeed an fr-controller of $S$, as already claimed in Example 4.1 .

Inclusion of the stop- and dead-traces of open nets defines a refinement relation. With the next theorem, we prove that an open net Impl fr-accords with an open net Spec if and only if every stop-trace of $I m p l$ is contained in the stoptraces of $S p e c$ and every dead-trace of $I m p l$ is contained in the dead-traces of Spec. In other words, we provide a trace-based characterization of $f r$-accordance.

Theorem 4.7 ( $f r$-accordance and stopdead-inclusion coincide). For two interface-equivalent open nets Impl and Spec, we have

$$
\begin{aligned}
& \text { Impl } \sqsubseteq_{f r, a c c} \text { Spec } \quad \text { iff } \quad \text { stop }(\operatorname{Impl}) \subseteq \operatorname{stop}(\text { Spec }) \text { and } \\
& \operatorname{dead}(\operatorname{Impl}) \subseteq \operatorname{dead}(\text { Spec }) .
\end{aligned}
$$

Proof. $\Leftarrow:$ Proof by contraposition. Consider an open net $C$ such that $\operatorname{Impl} \oplus$ $C$ and, equivalently, Spec $\oplus C$ are closed nets. Assume that $C$ is not an $f r$ controller of Impl. Then Impl and $C$ are not $f$-responsive by Definition 4.2 , 
and we find a trace $w \in \operatorname{stop}(\operatorname{Impl}) \cap \operatorname{dead}(C)$ or $w \in \operatorname{dead}(\operatorname{Impl}) \cap \operatorname{stop}(C)$ by Proposition 4.6. Due to stopdead-inclusion, we have $w \in \operatorname{stop}(\operatorname{Spec}) \cap \operatorname{dead}(C)$ and $w \in \operatorname{dead}($ Spec $) \cap \operatorname{stop}(C)$, respectively. Again with Proposition 4.6. Spec and $C$ are not $f$-responsive; that is, $C$ is not an $f r$-controller of Spec.

$\Rightarrow$ : Let $I$ be the input and $O$ be the output places of $I m p l$ and, equivalently, of Spec. If $I=O=\emptyset$, we have stop (Impl) $=\{\varepsilon\}=\operatorname{stop}($ Spec $)$. Furthermore, either $\operatorname{dead}(\operatorname{Impl})=\emptyset$ (and we are done) or $\operatorname{dead}(\operatorname{Impl})=\{\varepsilon\}$ and we consider an open net $C$ just consisting of a marked place, giving a final marking; $C$ is not a controller of $I m p l$, hence not of Spec, implying $\operatorname{dead}($ Spec $)=\{\varepsilon\}$.

For the case $I \neq \emptyset \neq O$, we consider a trace $w \in \operatorname{dead}(\operatorname{Impl})$. Let $w=$ $w_{1} \ldots w_{n}$ with $w_{j} \in I \uplus O$, for $j=1, \ldots, n$, and let $o \in I$ be arbitrary but fixed. We define an open net $N_{w, o}=\left(P^{\prime}, T^{\prime}, F^{\prime}, m_{0}^{\prime}, O, I, \Omega\right)$ exactly as in the proof of Theorem 3.8 see Fig. 5. As there, we see that a marking of env $\left(N_{w, o}\right)$ is a reachable stop except for inputs if and only if it is the only final marking $\left[p_{n}, p\right]$ $\left(1^{\prime}\right)$, i.e., $\operatorname{dead}\left(N_{w, o}\right)=\emptyset$.

Obviously, $\operatorname{Impl} \oplus N_{w, o}$ as well as $\operatorname{Spec} \oplus N_{w, o}$ are closed nets by construction of $N_{w, o}$. Because $w \in \operatorname{stop}\left(N_{w, o}\right)$ according to observation (1'), Impl and $N_{w, o}$ are not $f$-responsive by Proposition 4.6 and choice of $w$. Hence, $N_{w, o}$ is not an $f r$ controller of Impl and neither an $f r$-controller of Spec, because Impl fr-accords with Spec. Thus, Spec and $N_{w, o}$ are not $f$-responsive because of Definition 4.2 . Again with Proposition 4.6 and Definition 4.3 , there exists $v \in(I \uplus O)^{*}$ with

$$
m_{\text {env }(\text { Spec })} \stackrel{v}{\Longrightarrow} m_{1} \text { and } m_{e n v\left(N_{w, o}\right)} \stackrel{v}{\Longrightarrow} m_{2}
$$

such that both $m_{1}$ and $m_{2}$ are stops except for inputs, and additionally $m_{1}$ or $m_{2}$ is dead except for inputs. As $\operatorname{dead}\left(N_{w, o}\right)=\emptyset, m_{1}$ is dead except for inputs of env(Spec); furthermore, $m_{2}=\left[p_{n}, p\right]$.

As in the proof of Theorem 3.8, we derive

$$
m_{\text {env }(\text { Spec })} \stackrel{w}{\Longrightarrow} m_{1} \text { and } m_{\text {env }\left(N_{w, o}\right)} \stackrel{w}{\Longrightarrow} m_{2}
$$

and therefore $w \in \operatorname{dead}($ Spec $)$.

For a trace $w \in \operatorname{stop}(\operatorname{Impl})$, we fix some arbitrary $o \in I$ and define an open net $N_{w, o}^{\prime}=\left(P^{\prime}, T^{\prime}, F^{\prime}, m_{0}^{\prime}, O, I, \emptyset\right)$, which is identical to $N_{w, o}$ except for its empty set of final markings. Thus, $w \in \operatorname{dead}\left(N_{w, o}^{\prime}\right)$, and we succeed with an argumentation similar to the previous one.

Example 4.4. In Example 3.4 we showed that $\operatorname{stop}\left(S^{\prime}\right) \subseteq \operatorname{stop}(S)$. Each $w \in$ $\operatorname{stop}\left(S^{\prime}\right)$ can reach the nonfinal marking $\left[p_{1}\right]$ in $S^{\prime}$ and in $S$, hence $\operatorname{stop}\left(S^{\prime}\right)=$ $\operatorname{dead}\left(S^{\prime}\right)$ and $\operatorname{stop}\left(S^{\prime}\right) \subseteq \operatorname{dead}(S)$ (see Examples 3.4 and 4.2 . Therefore, $S^{\prime}$ fr-accords with $S$.

As shown in Example 4.1, $f r$-accordance is not a precongruence with respect to open net composition $\oplus$. Therefore, we characterize the coarsest precongruence, which is contained in $f r$-accordance in the following. 


\subsection{Deriving the coarsest precongruence for final-responsiveness}

The notion of $f$-responsiveness distinguishes between final and nonfinal markings. This information is needed to determine whether a marking is dead except for inputs. As we cannot derive this information from the $\mathcal{F}^{+}$-semantics, we must enhance it. The idea is basically to add an additional ingredient to a tree failure $(w, X)$ yielding a triple $(w, X, Y)$. This ingredient is a set $Y$, collecting traces that cannot lead the net to a final marking -including traces that cannot be performed at all. We bind the traces in $Y$ to a certain marking $m$ that is reached by executing $w$. Different markings $m$ can be reached by $w$ because of nondeterminism, so different sets $Y$ may be assigned to them. This construction ensures that we can identify traces in $\operatorname{dead}(N)$.

Definition $4.8\left(\mathcal{F}_{f i n}^{+}\right.$-semantics $)$. The $\mathcal{F}_{f i n}^{+}$-semantics of a labeled net $N$ is a set of fintree failures and defined as

$$
\begin{aligned}
\mathcal{F}_{\text {fin }}^{+}(N)=\{(w, X, Y) & \in \Sigma^{*} \times \mathcal{P}\left(\Sigma^{+}\right) \times \mathcal{P}\left(\Sigma^{*}\right) \mid \\
& \exists m \in M_{N}: m_{N} \stackrel{w}{\Longrightarrow} m \\
& \wedge \forall x \in X: m \not \\
& \left.\wedge \forall y \in Y: \forall m^{\prime}: m \stackrel{y}{\Longrightarrow} m^{\prime} \text { implies } m^{\prime} \notin \Omega_{N}\right\} .
\end{aligned}
$$

We say that after executing $w, N$ refuses $X$ and fin-refuses $Y$.

Example 4.5. We can distinguish the open nets $S$ and $S^{\prime}$ in Fig. 1 a and 3 by their $\mathcal{F}_{f i n}^{+}$-semantics: We have $\left(\varepsilon, \emptyset,(t r)^{*} e\right) \notin \mathcal{F}_{\text {fin }}^{+}(S)$; that is, after executing $\varepsilon$ reaching $\left[p_{0}\right],[e]$, or $\left[p_{1}, t\right]$ we can always perform some $w \in(t r)^{*} r$ and reach a final marking. However, $\left(\varepsilon, \emptyset,(t r)^{*} e\right) \in \mathcal{F}_{f i n}^{+}\left(S^{\prime}\right)$ because env $\left(S^{\prime}\right)$ can never perform $e$.

The $\mathcal{F}_{f i n}^{+}$-refinement relation is similarly defined as the $\mathcal{F}^{+}$-refinement relation in Definition 3.14 by closing up under an ordering over the fintree failures in $\mathcal{F}_{\text {fin }}^{+}$; this removes the too detailed information about the moment of choice in an open net.

Definition 4.9 $\left(\mathcal{F}_{f i n}^{+}\right.$-refinement). For two interface-equivalent labeled nets Impl and Spec, Impl $\mathcal{F}_{f i n}^{+}$-refines Spec, denoted by Impl $\sqsubseteq_{\mathcal{F}_{f i n}^{+}}$Spec, if

$$
\begin{aligned}
& \forall(w, X, Y) \in \mathcal{F}_{\text {fin }}^{+}(\operatorname{Impl}): \\
& \quad \exists x \in\{\varepsilon\} \cup \downarrow X \cup \downarrow Y:\left(w x, x^{-1} X, x^{-1} Y\right) \in \mathcal{F}_{f i n}^{+}(\text {Spec }) .
\end{aligned}
$$

For two interface-equivalent open nets $I m p l$ and $S p e c$, we define $I m p l \sqsubseteq_{\mathcal{F}_{\text {fin }}^{+}} S p e c$, if $\operatorname{env}(\operatorname{Impl}) \sqsubseteq_{\mathcal{F}_{\text {fin }}^{+}} \operatorname{env}($ Spec $)$.

Example 4.6. We have $\left(\varepsilon, \emptyset,(t r)^{*} e\right) \in \mathcal{F}_{\text {fin }}^{+}\left(S^{\prime}\right)$ by Example 4.5 . For all $w \in(t r)^{*}$, we have $\left(w, \emptyset,(t r)^{*} e\right) \notin \mathcal{F}_{\text {fin }}^{+}(S)$ : After $w$, we reach any of the markings $\left[p_{0}\right]$, $\left[p_{1}, t\right]$, or $[e]$ from which we can always reach the final marking [] with trace $e$ or tre. Furthermore, for all $v \in(t r)^{*} t$, we have $\left(v, \emptyset, r(t r)^{*} e\right) \notin \mathcal{F}_{f i n}^{+}(S)$ : After $v$, we are in the marking $\left[p_{1}\right]$ from which we reach the final marking [] with trace re. Thus, $S^{\prime}$ does not $\mathcal{F}_{\text {fin }}^{+}$-refine $S$. 
We will now show that $\mathcal{F}_{f i n}^{+}$-refinement is a precongruence for the composition operator $\oplus$. The proof will turn out to be fairly difficult. As in Sect. 3.2, we relate $\oplus$ to $\Uparrow$ on labeled nets and use that $\Uparrow$ is $\|$ followed by hiding of common actions.

If we consider the composition of two open nets $N_{1}$ and $N_{2}$, then its $\mathcal{F}_{\text {fin }}^{+}$ semantics coincides with that of the parallel composition of the two environments, env $\left(N_{1}\right)$ and $\operatorname{env}\left(N_{2}\right)$.

Lemma 4.10. For two composable open nets $N_{1}$ and $N_{2}$, we have

$$
\mathcal{F}_{\text {fin }}^{+}\left(\operatorname{env}\left(N_{1} \oplus N_{2}\right)\right)=\mathcal{F}_{\text {fin }}^{+}\left(\operatorname{env}\left(N_{1}\right) \Uparrow \operatorname{env}\left(N_{2}\right)\right) .
$$

Proof. This lemma follows directly from Lemma 2.12 If one net has a fintree failure $(w, X, Y)$ due to a marking $m$, then the other net can reach an agreeing marking $m^{\prime}$ with the trace $w$. If a trace $x \in X$ could be performed from $m^{\prime}$ in the second net, this would also be possible from $m$ in the first net due to bisimilarity, yielding a contradiction.

If a final marking $m_{1}^{\prime}$ could be reached from $m^{\prime}$ by performing $y \in Y$, then an agreeing $m_{1}$ can be reached from $m$. In the second net, all merged interface places $p$ or their derived $p^{i}$ and $p^{o}$ are empty at $m_{1}^{\prime}$, as they are at $m_{1}$. Hence, $m_{1}$ and $m_{1}^{\prime}$ coincide on the common places and $m_{1}$ is final. This is a contradiction, and $(w, X, Y)$ is also a fintree failure of the second net.

Next, we show how to determine the $\mathcal{F}_{\text {fin }}^{+}$-semantics for the composition of two labeled nets. Here and below, we use $\pi_{1}(w)$ and $\pi_{2}(w)$ to denote $\left.w\right|_{\Sigma_{1}}$ and $\left.w\right|_{\Sigma_{2}}$ for labeled nets $N_{1}$ and $N_{2}$ with alphabets $\Sigma_{1}$ and $\Sigma_{2}$. For a labeled net $N / A$ with $A \subseteq \Sigma, \phi(w)$ denotes $\left.w\right|_{\Sigma \backslash A}$. We canonically extend the notions of $\pi_{i}(w)$ and $\phi(w)$ pointwise to sets of traces.

Lemma $4.11\left(\mathcal{F}_{f i n}^{+}\right.$-semantics for labeled net composition). For composable labeled nets $N_{1}$ and $N_{2}$, we have

$$
\begin{aligned}
\mathcal{F}_{\text {fin }}^{+}\left(N_{1} \| N_{2}\right)=\left\{\left(w, X_{1} \cup X_{2}, Y_{1} \cup Y_{2}\right) \mid\right. & \left(\pi_{1}(w), \pi_{1}\left(X_{1}\right), \pi_{1}\left(Y_{1}\right)\right) \in \mathcal{F}_{\text {fin }}^{+}\left(N_{1}\right), \\
& \left.\left(\pi_{2}(w), \pi_{2}\left(X_{2}\right), \pi_{2}\left(Y_{2}\right)\right) \in \mathcal{F}_{\text {fin }}^{+}\left(N_{2}\right)\right\} .
\end{aligned}
$$

Proof. $\subseteq$ : Let $E=N_{1} \| N_{2}$ and let $(w, X, Y)$ be a fintree failure of $E$. Then there exists a marking $m$ with $m_{E} \stackrel{w}{\Longrightarrow} m$ according to Definition 4.8. Applying Proposition 2.8, we can project $w$ onto $w_{1}=\pi_{1}(w)$ and $w_{2}=\pi_{2}(w)$ such that $w \in w_{1} \| w_{2}, m_{N_{1}}=\left.\left.m_{E}\right|_{P_{1}} \stackrel{w_{1}}{\Longrightarrow} m\right|_{P_{1}}$, and $m_{N_{2}}=\left.\left.m_{E}\right|_{P_{2}} \stackrel{w_{2}}{\Longrightarrow} m\right|_{P_{2}}$. For each $x \in X$, we have $\left.m\right|_{P_{1}} \stackrel{\pi_{1}(x)}{\rightleftharpoons}$ or $\left.m\right|_{P_{2}} \stackrel{\pi_{2}(x)}{\Longrightarrow}$ by Proposition 3.11 . Set $X_{1}$ consists of the $x$ with the first and set $X_{2}$ of the $x$ with the second property, where $X_{1}$ and $X_{2}$ might overlap. Likewise, we can subdivide $Y$ by Proposition 2.8. Note that if a $y \in Y$ can be performed at $m$ at all, then it does not reach a final marking; that is, neither $\pi_{1}(y)$ nor $\pi_{2}(y)$ ever reach a final marking. Thus, no trace in $\pi_{1}\left(X_{1}\right)$ can be performed from $\left.m\right|_{P_{1}}$ and no trace in $\pi_{1}\left(Y_{1}\right)$ can reach a final marking from $\left.m\right|_{P_{1}}$. Hence, $\left(\pi_{1}(w), \pi_{1}\left(X_{1}\right), \pi_{1}\left(Y_{1}\right)\right) \in \mathcal{F}_{\text {fin }}^{+}\left(N_{1}\right)$, and similarly for $N_{2}$.

$\supseteq$ : Likewise, given $\left(w, X_{1} \cup X_{2}, Y_{1} \cup Y_{2}\right)$ with $\left(\pi_{1}(w), \pi_{1}\left(X_{1}\right), \pi_{1}\left(Y_{1}\right)\right) \in \mathcal{F}_{\text {fin }}^{+}\left(N_{1}\right)$ and $\left(\pi_{2}(w), \pi_{1}\left(X_{2}\right), \pi_{1}\left(Y_{2}\right)\right) \in \mathcal{F}_{\text {fin }}^{+}\left(N_{2}\right)$ due to $m_{1}$ and $m_{2}$, one finds that $w$ is a trace of $E$ reaching $m_{1}+m_{2}$, which justifies the first fintree failure. 
From the $\mathcal{F}^{+}$-semantics in Proposition 3.12 , we adapt the following construction of $\mathcal{F}_{\text {fin }}^{+}(N / A)$.

Lemma 4.12. For any labeled net $N$ and any label set $A \subseteq \Sigma_{N}^{*}$, we have

$$
\mathcal{F}_{\text {fin }}^{+}(N / A)=\left\{(\phi(w), X, Y) \mid\left(w, \phi^{-1}(X), \phi^{-1}(Y)\right) \in \mathcal{F}_{\text {fin }}^{+}(N)\right\} .
$$

The next proposition yields the $\mathcal{F}_{\text {fin }}^{+}$-semantics for the composition of two open nets.

Proposition $4.13\left(\mathcal{F}_{f i n}^{+}\right.$-semantics for open net composition). For composable open nets $N_{1}$ and $N_{2}$, we have

$$
\begin{aligned}
\mathcal{F}_{f i n}^{+}\left(N_{1} \oplus N_{2}\right)=\{(w, X, Y) \mid & \exists\left(w_{1}, X_{1}, Y_{1}\right) \in \mathcal{F}_{\text {fin }}^{+}\left(N_{1}\right),\left(w_{2}, X_{2}, Y_{2}\right) \in \mathcal{F}_{f i n}^{+}\left(N_{2}\right): \\
w \in w_{1} \Uparrow w_{2} \wedge \forall x \in X, y \in Y: & \left(x \in x_{1} \Uparrow x_{2} \text { implies } x_{1} \in X_{1} \vee x_{2} \in X_{2}\right) \\
\wedge & \left.\left(y \in y_{1} \Uparrow y_{2} \text { implies } y_{1} \in Y_{1} \vee y_{2} \in Y_{2}\right)\right\} .
\end{aligned}
$$

Proof. Follows the same argumentation as the proof of Proposition 3.13 , because the sets $X$ and $Y$ are treated analogously.

Having characterized the $\mathcal{F}_{f i n}^{+}$-semantics for composition and hiding, we shall show that $\mathcal{F}_{\text {fin }}^{+}$-refinement is a precongruence for the composition operator $\oplus$. First, we show the precongruence result for labeled nets and operator $\|$. Then, we show that this result is also preserved under hiding. Finally, we combine these results to show the precongruence for open nets and the operator $\oplus$.

For the first and second step, we can build upon the proof ideas introduced for should testing in [29, Lemma 46], where saturation conditions like SAT1-3 below are employed. The key idea in [29] is to shift traces from the refusal set of Impl. We apply the same proof strategy for the $X$-part of the fintree failures, which is closed under suffix (SAT3). Because this does not hold for the $Y$-part, we cannot directly apply this idea here. We overcome this problem by adding the set $X$ to the set $Y$, thereby using the fourth of the following saturation conditions on fintree failures.

SAT1: $(w, X, Y) \in \mathcal{F}_{f i n}^{+}(N), X^{\prime} \subseteq X, Y^{\prime} \subseteq Y$ implies $\left(w, X^{\prime}, Y^{\prime}\right) \in \mathcal{F}_{\text {fin }}^{+}(N)$

SAT2: $(w, X, Y) \in \mathcal{F}_{\text {fin }}^{+}(N) \wedge \forall z \in Z:\left(w z, z^{-1} X, z^{-1} Y\right) \notin \mathcal{F}_{\text {fin }}^{+}(N)$ implies $(w, X \cup Z, Y \cup Z) \in \mathcal{F}_{\text {fin }}^{+}(N)$

SAT3: $(w, X, Y) \in \mathcal{F}_{\text {fin }}^{+}(N)$ implies $(w, \uparrow X, Y) \in \mathcal{F}_{\text {fin }}^{+}(N)$

SAT4: $(w, X, Y) \in \mathcal{F}_{\text {fin }}^{+}(N)$ implies $(w, X, X \cup Y) \in \mathcal{F}_{\text {fin }}^{+}(N)$

SAT1 states that, given a fintree failure $(w, X, Y)$, the sets $X$ and $Y$ can be arbitrarily decreased and the resulting triple is again a fintree failure. Furthermore, the refusal part of $\mathcal{F}_{\text {fin }}^{+}$is saturated in the sense that the sets $X$ and $Y$ can be extended by any set of traces $z$ such that $\left(w z, z^{-1} X, z^{-1} Y\right) \notin \mathcal{F}_{\text {fin }}^{+}(N)$ (SAT2). SAT3 states that the $X$-part is closed under suffix, and SAT4 shows that the refusal part of $\mathcal{F}_{\text {fin }}^{+}$is saturated in the sense that the set $X$ can be added to $Y$.

SAT1, SAT3 and SAT4 are obvious; to see SAT2, assume that some $z$ could be performed from the marking $m$ justifying the tree failure $(w, X, Y)$. 
Remark 4.1. We have also explored the idea to encode each $w \in Y$ by $w \checkmark$ for a new symbol $\checkmark$. Then, one can add the resulting traces to $X$ and work with something that looks like an ordinary tree failure. The hope was that this would allow us to use the result [29, Lemma 46] instead of its proof idea, but we have not managed to show the necessary saturation conditions for the domain used in $[29$.

Lemma 4.14. $\mathcal{F}_{\text {fin }}^{+}$-refinement is a precongruence for labeled nets for the composition operator $\|$.

Proof. Let $I m p l$ and $S p e c$ be interface-equivalent open nets, and let open net $C$ be composable with Spec. Let further $\operatorname{Impl} \sqsubseteq_{\mathcal{F}+}$ Spec. We show that $\operatorname{Impl} \| C \sqsubseteq_{\mathcal{F}^{+}}$ Spec $\| C$, following to a large extent the proof of [29, Lemma 46]. For understandability, we also show the full proof for the case $\Sigma_{S p e c}=\Sigma_{C}$ here, because in this case the projection functions in the construction of the synchronized fintree failures become the identity over the complete alphabet and hence disappear.

Consider a fintree failure $\left(w, X_{\text {Impl }} \cup X_{C}, Y_{\text {Impl }} \cup Y_{C}\right) \in \mathcal{F}_{\text {fin }}^{+}(\operatorname{Impl} \| C)$ such that $\left(w, X_{\text {Impl }}, Y_{\text {Impl }}\right) \in \mathcal{F}_{\text {fin }}^{+}(I m p l)$ and $\left(w, X_{C}, Y_{C}\right) \in \mathcal{F}_{\text {fin }}^{+}(C)$. Define the set

$$
W=\left\{v \mid\left(w v, v^{-1} X_{C}, v^{-1} Y_{C}\right) \notin \mathcal{F}_{\text {fin }}^{+}(C)\right\}
$$

which contains those traces that can be added to $X_{C}$ and $Y_{C}$ according to SAT2. We shift the traces in $W$ from $I m p l$ to $C$. To this end, we define four sets

$$
\begin{aligned}
X_{\text {Impl }}^{\prime} & =X_{\text {Impl }} \backslash \uparrow W, \\
Y_{\text {Impl }}^{\prime} & =Y_{\text {Impl }} \backslash \uparrow W, \\
X_{C}^{\prime} & =X_{C} \cup \uparrow W, \\
Y_{C}^{\prime} & =Y_{C} \cup \uparrow W .
\end{aligned}
$$

We immediately see: $X_{\text {Impl }} \cup X_{C} \subseteq X_{\text {Impl }}^{\prime} \cup X_{C}^{\prime}$ as well as $Y_{\text {Impl }} \cup Y_{C} \subseteq$ $Y_{\text {Impl }}^{\prime} \cup Y_{C}^{\prime}$ and $X_{\text {Impl }}^{\prime} \cup Y_{\text {Impl }}^{\prime} \subseteq X_{\text {Impl }} \cup Y_{\text {Impl }} \cup X_{C} \cup Y_{C}$. By SAT1, we have $\left(w, X_{\text {Impl }}^{\prime}, Y_{\text {Impl }}^{\prime}\right) \in \mathcal{F}_{\text {fin }}^{+}(I m p l)$.

Due to Impl $\mathcal{F}^{+}$Spec, there exists $x \in\{\varepsilon\} \cup \downarrow X_{\text {Impl }}^{\prime} \cup \downarrow Y_{\text {Impl }}^{\prime}$ such that

$$
\left(w x, x^{-1} X_{\text {Impl }}^{\prime}, x^{-1} Y_{\text {Impl }}^{\prime}\right) \in \mathcal{F}_{\text {fin }}^{+}(\text {Spec })
$$

We have $x \notin \uparrow W$. Assume the contrary: $x=\varepsilon$ implies $\varepsilon \in W$ which is a contradiction to the construction of $W . x \in \downarrow X_{\text {Impl }}^{\prime}$ implies $\exists x^{\prime} \in X_{\text {Impl }}^{\prime}: x \sqsubseteq$ $x^{\prime} \wedge \exists v \in W: v \sqsubseteq x \sqsubseteq x^{\prime}$ which is a contradiction to the definition of $X_{\text {Impl }}^{\prime}$. The same argument also applies to $x \in Y_{\text {Impl }}^{\prime}$.

From $x \notin W$, it follows that $\left(w x, x^{-1} X_{C}, x^{-1} Y_{C}\right) \in \mathcal{F}_{f i n}^{+}(C)$. Further, for all $u \in x^{-1} W$ (i.e., $x u \in W$ ), $\left(w x u, u^{-1} x^{-1} X_{C}, u^{-1} x^{-1} Y_{C}\right) \notin \mathcal{F}_{\text {fin }}^{+}(C)$.

By SAT2, $\left(w x, x^{-1}\left(X_{C} \cup W\right), x^{-1}\left(Y_{C} \cup W\right)\right) \in \mathcal{F}_{\text {fin }}^{+}(C)$. Consider now the second ingredient, $x^{-1}\left(X_{C} \cup W\right)$. By SAT3, this implies $\uparrow x^{-1}\left(X_{C} \cup W\right)$. With Lemma 2.14(3), we have $\uparrow x^{-1}\left(X_{C} \cup W\right) \supseteq x^{-1}\left(X_{C} \cup \uparrow W\right)=x^{-1} X_{C}^{\prime}$. Now, according to SAT1, $x^{-1} X_{C}^{\prime}$ can replace $x^{-1}\left(X_{C} \cup W\right)$. 
Consider now the third ingredient, $x^{-1}\left(Y_{C} \cup W\right)$. By SAT4, we extend this set to $x^{-1}\left(Y_{C} \cup W\right) \cup x^{-1} X_{C}^{\prime} \supseteq x^{-1} \uparrow W \cup x^{-1} Y_{C}=x^{-1}\left(\uparrow W \cup Y_{C}\right)=x^{-1} Y_{C}^{\prime}$. Now, SAT1 allows that $x^{-1} Y_{C}^{\prime}$ can replace $x^{-1}\left(Y_{C} \cup W\right)$.

Combining these results, we get $\left(w x, x^{-1} X_{C}^{\prime}, x^{-1} Y_{C}^{\prime}\right) \in \mathcal{F}_{\text {fin }}^{+}(C)$. Then, by Lemma 4.11 and (1), we obtain $\left(w x, x^{-1}\left(X_{\text {Impl }}^{\prime} \cup X_{C}^{\prime}\right), x^{-1}\left(Y_{\text {Impl }}^{\prime} \cup Y_{C}^{\prime}\right)\right) \in$ $\mathcal{F}_{\text {fin }}^{+}($Spec $\| C)$. By SAT1, we have $\left(w x, x^{-1}\left(X_{\text {Impl }} \cup X_{C}\right), x^{-1}\left(Y_{\text {Impl }} \cup Y_{C}\right)\right) \in$ $\mathcal{F}_{\text {fin }}^{+}($Spec $\| C)$ where $x \in\left(\{\varepsilon\} \cup \downarrow X_{\text {Impl }}^{\prime} \cup \downarrow Y_{\text {Impl }}^{\prime}\right) \subseteq\left(\{\varepsilon\} \cup \downarrow X_{\text {Impl }} \cup \downarrow Y_{\text {Impl }} \cup\right.$ $\left.\downarrow X_{C} \cup \downarrow Y_{C}\right)$.

Now consider the general case. Let $\pi$ and $\pi_{C}$ denote projections, projecting onto the alphabets $\Sigma_{S p e c}=\Sigma_{I m p l}$ and $\Sigma_{C}$, respectively. We have

1. $\pi(V \cup W)=\pi(V) \cup \pi(W)$

2. $\pi(\uparrow V) \subseteq \uparrow \pi(V)$

3. $\pi\left(w^{-1} V\right) \subseteq \pi(w)^{-1} \pi(V)$

Consider a fintree failure $\left(w, X_{\text {Impl }} \cup X_{C}, Y_{\text {Impl }} \cup Y_{C}\right) \in \mathcal{F}_{\text {fin }}^{+}(\operatorname{Impl} \| C)$ such that $\left(\pi(w), \pi\left(X_{\text {Impl }}\right), \pi\left(Y_{\text {Impl }}\right)\right) \in \mathcal{F}_{\text {fin }}^{+}(\operatorname{Impl})$ and $\left(\pi_{C}(w), \pi_{C}\left(X_{C}\right), \pi_{C}\left(Y_{C}\right)\right) \in$ $\mathcal{F}_{\text {fin }}^{+}(C)$. Define the set

$$
W=\left\{v \mid\left(\pi_{C}(w v), \pi_{C}\left(v^{-1} X_{C}\right), \pi_{C}\left(v^{-1} Y_{C}\right)\right) \notin \mathcal{F}_{\text {fin }}^{+}(C)\right\} .
$$

We shift the traces in $W$ from $\operatorname{Impl}$ to $C$. To this end, we define four sets

$$
\begin{aligned}
X_{\text {Impl }}^{\prime} & =X_{\text {Impl }} \backslash \uparrow W, \\
Y_{\text {Impl }}^{\prime} & =Y_{\text {Impl }} \backslash \uparrow W, \\
X_{C}^{\prime} & =X_{C} \cup \uparrow W, \\
Y_{C}^{\prime} & =Y_{C} \cup \uparrow W .
\end{aligned}
$$

We immediately see: $X_{\text {Impl }} \cup X_{C} \subseteq X_{\text {Impl }}^{\prime} \cup X_{C}^{\prime}$ as well as $Y_{\text {Impl }} \cup Y_{C} \subseteq Y_{\text {Impl }}^{\prime} \cup Y_{C}^{\prime}$ and $X_{\text {Impl }}^{\prime} \cup Y_{\text {Impl }}^{\prime} \subseteq X_{\text {Impl }} \cup Y_{\text {Impl }} \cup X_{C} \cup Y_{C}$. By SAT1, we have $\left(\pi(w), \pi\left(X_{\text {Impl }}^{\prime}\right), \pi\left(Y_{\text {Impl }}^{\prime}\right)\right) \in \mathcal{F}_{\text {fin }}^{+}(\operatorname{Impl})$.

Because of Impl $\sqsubseteq_{\mathcal{F}+}$ Spec, there exists $x \in\{\varepsilon\} \cup \downarrow X_{\text {Impl }}^{\prime} \cup \downarrow Y_{\text {Impl }}^{\prime}$ such that $\left(\pi(w x), \pi(x)^{-1} \pi\left(X_{\text {Impl }}^{\prime}\right), \pi(x)^{-1} \pi\left(Y_{\text {Impl }}^{\prime}\right)\right) \in \mathcal{F}_{\text {fin }}^{+}($Spec $)$. Hence, we have

$$
\left(\pi(w x), \pi\left(x^{-1} X_{\text {Impl }}^{\prime}\right), \pi\left(x^{-1} Y_{\text {Impl }}^{\prime}\right)\right) \in \mathcal{F}_{\text {fin }}^{+}(\text {Spec })
$$

due to Item 3 and SAT1.

Again, trace $x \notin \uparrow W$ (by the same argumentation as in the proof of the case $\left.\Sigma_{S p e c}=\Sigma_{C}\right)$, and we conclude that $\left(\pi_{C}(w x), \pi_{C}\left(x^{-1} X_{C}\right), \pi_{C}\left(x^{-1} Y_{C}\right)\right) \in$ $\mathcal{F}_{\text {fin }}^{+}(C)$. Further, for all $u \in x^{-1} W$ (i.e., $x u \in W$ ), $\left(\pi_{C}(w x u), \pi_{C}\left(u^{-1} x^{-1} X_{C}\right), \pi_{C}\left(u^{-1} x^{-1} Y_{C}\right)\right) \notin \mathcal{F}_{\text {fin }}^{+}(C)$, due to the definition of $W$.

Now, $\left(\pi_{C}(w x u), \pi_{C}(u)^{-1} \pi_{C}\left(x^{-1} X_{C}\right), \pi_{C}(u)^{-1} \pi_{C}\left(x^{-1} Y_{C}\right)\right) \notin \mathcal{F}_{\text {fin }}^{+}(C)$ with Item 3 and SAT1, and we obtain, by Item 1 and SAT2,

$$
\left(\pi_{C}(w x), \pi_{C}\left(x^{-1}\left(X_{C} \cup W\right)\right), \pi_{C}\left(x^{-1}\left(Y_{C} \cup W\right)\right)\right) \in \mathcal{F}_{\text {fin }}^{+}(C) .
$$


Consider the second ingredient, $\pi_{C}\left(x^{-1}\left(X_{C} \cup W\right)\right)$ of this fintree failure. Applying SAT3, we obtain $\uparrow \pi_{C}\left(x^{-1}\left(X_{C} \cup W\right)\right)$ and with SAT1 and Item 2, $\pi_{C}\left(\uparrow x^{-1}\left(X_{C} \cup W\right)\right)$. Because $x \notin \uparrow W$, we can apply Lemma 2.14 (3) and SAT1, and we arrive at $\pi_{C}\left(x^{-1}\left(X_{C} \cup \uparrow W\right)\right)=\pi_{C}\left(x^{-1} X_{C}^{\prime}\right)$.

For the third ingredient $\pi_{C}\left(x^{-1}\left(Y_{C} \cup W\right)\right)$ of this fintree failure, we obtain by SAT4 $\pi_{C}\left(x^{-1}\left(Y_{C} \cup W\right)\right) \cup \pi_{C}\left(x^{-1} X_{C}^{\prime}\right)$. By Item 1, we can transform this into $\pi_{C}\left(x^{-1}\left(Y_{C} \cup W \cup X_{C}^{\prime}\right)\right)$ and by SAT1 into $\pi_{C}\left(x^{-1}\left(Y_{C} \cup \uparrow W\right)\right)=\pi_{C}\left(x^{-1} Y_{C}^{\prime}\right)$.

Combining these results yields $\left(\pi_{C}(w x), \pi_{C}\left(x^{-1} X_{C}^{\prime}\right), \pi_{C}\left(x^{-1} Y_{C}^{\prime}\right)\right) \in \mathcal{F}_{f i n}^{+}(C)$. Then, with Lemma 4.11 and (2), we obtain

$$
\left(w x, x^{-1}\left(X_{\text {Impl }}^{\prime} \cup X_{C}^{\prime}\right), x^{-1}\left(Y_{\text {Impl }}^{\prime} \cup Y_{C}^{\prime}\right)\right) \in \mathcal{F}_{\text {fin }}^{+}(\text {Spec } \| C) .
$$

Applying SAT1 yields $\left(w x, x^{-1}\left(X_{\text {Impl }} \cup X_{C}\right), x^{-1}\left(Y_{\text {Impl }} \cup Y_{C}\right) \in \mathcal{F}_{\text {fin }}^{+}(\right.$Spec $\| C)$ where $x \in\left(\{\varepsilon\} \cup \downarrow X_{\text {Impl }}^{\prime} \cup \downarrow Y_{\text {Impl }}^{\prime}\right) \subseteq\left(\{\varepsilon\} \cup \downarrow X_{\text {Impl }} \cup \downarrow Y_{I m p l} \cup \downarrow X_{C} \cup \downarrow Y_{C}\right)$.

Remark 4.2. The proof of Lemma 4.14 is not restricted to sets of fintree failures of labeled nets, but holds for general sets of fintree failures for which the four saturation SAT1 - SAT4 hold.

We show that $\mathcal{F}_{\text {fin }}^{+}$-refinement for labeled nets is preserved under hiding.

Lemma 4.15. $\mathcal{F}_{\text {fin }}^{+}$-refinement for labeled nets is preserved under hiding.

Proof. Let Impl and Spec be interface-equivalent labeled nets such that Impl $\sqsubseteq_{\mathcal{F}_{f i n}^{+}}$Spec. Further, let $A \subseteq \Sigma^{*}$ and $(w, X, Y) \in \mathcal{F}_{\text {fin }}^{+}(\operatorname{Impl} / A)$. Consider $\left(v, \phi^{-1}(X), \phi^{-1}(Y)\right) \in \mathcal{F}_{\text {fin }}^{+}(\operatorname{Impl})$ with $w=\phi(v)$. Because $\operatorname{Impl} \mathcal{F}_{\text {fin }}^{+}$-refines Spec, there is $x \in\{\varepsilon\} \cup \downarrow \phi^{-1}(X) \cup \downarrow \phi^{-1}(Y)$ with $\left(v x, x^{-1} \phi^{-1}(X), x^{-1} \phi^{-1}(Y)\right) \in$ $\mathcal{F}_{\text {fin }}^{+}($Spec $)$. It can be shown that $\phi^{-1}\left(\phi(x)^{-1} X\right)=x^{-1} \phi^{-1}(X)$. Using this observation together with $\left(v, \phi^{-1}(X), \phi^{-1}(Y)\right) \in \mathcal{F}_{\text {fin }}^{+}(\operatorname{Impl})$, we conclude that $\left(\phi(v x), \phi(x)^{-1} X, \phi(x)^{-1} Y\right) \quad \in \quad \mathcal{F}_{\text {fin }}^{+}($Spec $/ A) \quad$ and additionally $\left(\phi(v) \phi(x), \phi(x)^{-1} X, \phi(x)^{-1} Y\right) \in \mathcal{F}_{\text {fin }}^{+}($Spec $/ A)$. Because $\phi(v)=w$ and $\phi(x) \in$ $\{\varepsilon\} \cup \downarrow(X) \cup \downarrow(Y)$, the lemma holds.

With Lemma 4.14 and Lemma 4.15 , we have the ingredients to show that $\mathcal{F}_{\text {fin }}^{+}$-refinement is also a precongruence for the composition operator $\oplus$.

Theorem 4.16 (precongruence). $\mathcal{F}_{\text {fin }}^{+}$-refinement is a precongruence for the composition operator $\oplus$.

Proof. The proof is analogous to the proof of Theorem 3.15 By Lemma 4.14 and Lemma $4.15 \mathcal{F}_{f i n}^{+}$-refinement is a precongruence for the composition operator $\Uparrow$ and, by Lemma 4.10, also for for the composition operator $\oplus$.

With the next theorem, we show that $\mathcal{F}_{\text {fin }}^{+}$-refinement and the coarsest precongruence that is contained in the $f r$-accordance relation coincide. 
Theorem 4.17 (precongruence and $\mathcal{F}_{\text {fin }}^{+}$-refinement coincide). For two interface-equivalent open nets Impl and Spec, we have

$$
\text { Impl } \sqsubseteq_{\mathcal{F}_{f i n}^{+}} \text {Spec iff Impl } \sqsubseteq_{f r, a c c}^{c} \text { Spec. }
$$

Proof. $\Rightarrow$ : Consider a trace $w \in \operatorname{stop}(\operatorname{Impl})(w \in \operatorname{dead}(\operatorname{Impl}))$; we prove $w \in$ stop $($ Spec $)(w \in \operatorname{dead}(S p e c))$. Then, applying Theorem 4.7, we get Impl $\sqsubseteq_{f r, a c c}$ Spec, and this in turn also shows the claim with Theorem 4.16 and the definition of $\sqsubseteq_{f r, a c c}^{c}$. So let $O$ be the set of output places of $I m p l$ and of Spec.

We have $w \in \operatorname{stop}(\operatorname{Impl})$ iff $(w, O, \emptyset) \in \mathcal{F}_{\text {fin }}^{+}($Impl) by Definitions 3.4 and 3.9 . Then, by Impl $\Xi_{\mathcal{F}_{\text {fin }}^{+}}$Spec, there must be a suitable $x \in\{\varepsilon\} \cup O \equiv\{\varepsilon\} \cup \downarrow O$ that satisfies the defining condition of Definition 4.8. We cannot have $x \in O$ because $(w x,\{\varepsilon\}, \emptyset) \notin \mathcal{F}_{\text {fin }}^{+}($Spec $)$by Definition 4.8. Thus, $x=\varepsilon$ and $(w, O, \emptyset) \in$ $\mathcal{F}_{\text {fin }}^{+}($Spec $)$, implying $w \in \operatorname{stop}($ Spec $)$.

We have $w \in \operatorname{dead}(\operatorname{Impl})$ iff $(w, O,\{\varepsilon\}) \in \mathcal{F}_{\text {fin }}^{+}(\operatorname{Impl})$ by Definition 4.3. Again, $x=\varepsilon$ and thus $(w, O,\{\varepsilon\}) \in \mathcal{F}_{\text {fin }}^{+}($Spec $)$, implying $w \in \operatorname{dead}($ Spec $)$.

$\Leftarrow$ : Suppose Impl $\sqsubseteq_{f r, a c c}^{c}$ Spec, and let $(w, X, Y) \in \mathcal{F}_{\text {fin }}^{+}(\operatorname{Impl})$. In addition, consider an open net $C$ with the new output $x$ and the new input $y$. Open net $C$ has the empty initial marking and contains only a single transition that can indefinitely repeat to produce a token in $x$ while consuming a token from place $y$. In addition, its final marking is the empty marking. The idea is to construct an open net $N$ from $(w, X, Y)$ such that $C$ is not an $f r$-controller of $I m p l \oplus N$ because of $(w, X, Y)$. By Impl $\sqsubseteq_{f r, a c c}^{c}$ Spec and because $\sqsubseteq_{f r, a c c}^{c}$ is a precongruence, we have $\operatorname{Impl} \oplus N \sqsubseteq_{f r, a c c}^{c}$ Spec $\oplus N$ and thus $\operatorname{Impl} \oplus N \sqsubseteq_{f r, a c c}$ Spec $\oplus N$ by Definition 4.2. Hence, $C$ is also not an $f r$-controller of $S p e c \oplus N$, and from this we shall conclude that $(w, X, Y)$ is covered by $\mathcal{F}_{\text {fin }}^{+}($Spec $)$according to Definition 4.9 . Then we will have proved $\operatorname{Impl} \sqsubseteq_{\mathcal{F}_{\text {fin }}^{+}}$Spec.

The construction of the open net $N$ is similar to the one in the proof of (the reverse implication of) Theorem 3.17 the tree part on the right-hand side of Fig. 6 corresponds to $X \cup Y$ now. The open net $N$ has input places $I=$ $O_{\text {Impl }} \uplus\{x\}$, output places $O=I_{\text {Impl }} \uplus\{y\}$, and enables a transition sequence $v=t_{1} \ldots t_{k}$. Each transition in $v$ is connected to an interface place of $N$ such that the corresponding trace of interface actions is $w$; that is, the net $N$ contains net $N_{w}$ as in Fig. 5 . Thus, we can essentially fire the trace $w$ of $e n v(N)$ in $\operatorname{Impl} \oplus N$ and, therefore, in $\operatorname{Impl} \oplus N \oplus C$ by firing $v$ instead of the labeled transitions. This way, we reach in $I m p l$ a marking $m$ that refuses $X$ in $e n v(I m p l)$; in $N$, there is only one token in a place $p_{\varepsilon}$ and the token in a place $p$ has been consumed. This token is necessary to enable transition $t^{\prime}$ that is - together with transition $t$ essential for $f$-responsiveness, because they can repeatedly communicate with $C$. The place $p$ can only be marked again by firing some transition $t_{x}^{\prime}$ with $x \in X$, and this in turn requires the firing of a transition sequence that - similarly to $v$-looks to $I m p l$ like the trace $x$. But this trace cannot be fired at $m$. In addition, every trace $y \in Y$ that cannot lead to a final marking in Impl leads to a final marking in the tree part of $N$. This construction guarantees that there is a marking reachable in the composition $\operatorname{Impl} \oplus N \oplus C$ which is neither responsive 
(because place $p$ is not marked and hence there is no communication between $C$ and $N$ ) nor reaches a final marking (because if $N \oplus C$ is in a final marking, then Impl is not). As a consequence, $\operatorname{Impl} \oplus N \oplus C$ is not $f$-responsive and, thus, $C$ is not an $f r$-controller of $\operatorname{Impl} \oplus N$.

To achieve the effect just described, the second part of the open net $N$ encodes the tree part for $X$ and $Y$ of fintree failure $(w, X, Y)$; this second part is a tree representing $X \cup Y$. Common prefixes thereby correspond to the same path in this part. If a path corresponds to some $y \in Y$, a token on the place at the end of this path is a final marking of $N$; if for example $b \in Y$, then the marking with just one token on the place $p_{b}$ is final; see Fig. 6. For a path corresponding to some $x \in X$, a token in the respective place allows to mark $p$ again.

Let $w=w_{1} \ldots w_{k}$ such that for $j=1, \ldots, k, w_{j} \in I_{\text {Impl }} \uplus O_{\text {Impl }}$. Let $N=$ $\left(P, T, F, m_{N}, O, I, \Omega\right)$ be an open net like the one in the proof of Theorem 3.17 . but replace every occurrence of $\downarrow X$ with $\downarrow X \cup \downarrow Y$ and add $\Omega=\left\{\left[p_{z}\right] \mid z \in Y\right\}$.

As argued previously, we now have that $C$ is not an $f r$-controller of $\operatorname{Spec} \oplus N$; that is, some marking $m_{1}$ can be reached in $\operatorname{Spec} \oplus N \oplus C$ where $f$-responsiveness is violated. Clearly, places $p, x$, and $y$ must be empty in $m_{1}$; thus, $v$ has been fired in $N$ plus possibly some transitions in the fintree part of the net. There is just one token in the places of inner $(N)$, and it is in some $p_{u}$ with $u u^{\prime} \in X$ (resp. $u u^{\prime} \in Y$ ). Let $m_{2}$ be the projection of $m_{1}$ onto the places of Spec. From the point of view of Spec, we have fired a trace wu of env(Spec) reaching $m_{2}$. Because in Spec $\oplus N \oplus C$ no $t_{u u^{\prime}}^{\prime}$ can become enabled and the composition cannot reach a final marking - otherwise, $C$ would be an $f r$-controller-no $u^{\prime}$ can be fired in env(Spec) at $m_{2}$ and a final marking is not reachable. Thus, we conclude $\left(w u,\left\{u^{\prime} \mid u u^{\prime} \in X\right\},\left\{u^{\prime} \mid u u^{\prime} \in Y\right\}\right) \in \mathcal{F}_{\text {fin }}^{+}($Spec $)$and, therefore, $\operatorname{Impl} \sqsubseteq \mathcal{F}_{\text {fin }}^{+}$Spec.

Example 4.7. As shown in Example 4.1. $S^{\prime} \sqsubseteq_{f r, a c c}^{c} S$ does not hold. This is now confirmed with Theorem 4.17 because $S^{\prime}$ does not $\mathcal{F}_{\text {fin }}^{+}$-refine $S$ by Example 4.6 .

\section{Bounded nets without responsiveness}

In the two previous sections, we presented precongruences for responsiveness and $f$-responsiveness. The former notion does not take into account final markings whereas the latter notion does. In this section, we study another property of open net compositions: boundedness. We first consider boundedness in isolation - that is, without requiring open nets to be responsive or to have final markingsand add boundedness to responsiveness and $f$-responsiveness in the subsequent sections. That way, technicalities become simpler.

The next two definitions define the notion of a controller and of accordance for boundedness.

Definition 5.1 ( $b$-controller, $b$-accordance). An open net $C$ is a $b$-controller of an open net $N$ if their composition $N \oplus C$ is a $b$-bounded, closed net. 
For interface-equivalent open nets Impl and Spec, Impl b-accords with Spec, denoted by Impl $\sqsubseteq_{b, a c c}$ Spec, if for all open nets $C: C$ is a $b$-controller of Spec implies $C$ is a $b$-controller of $I m p l$.

Example 5.1. The open net $C^{\prime}$ in Fig. 7 a is a 1-controller of the open net $S$ in Fig. 1a because their composition $S \oplus C^{\prime}$ in Fig. $7 \mathrm{~b}$ is a 1-bounded closed net. Consequently, $C^{\prime}$ is a $b$-controller of $S$ for every $b \in \mathbb{N}_{+}$. In contrast, the open net $C$ in Fig. $1 \mathrm{~b}$ is not a $b$-controller of $S$ for any $b \in \mathbb{N}_{+}$because the place $r$ is unbounded in their composition $S \oplus C$ in Fig. 1c after the error has been caught.

While every open net has at least one $r$-controller and one $f r$-controller, there exist open nets that do not have any $b$-controller. An example is an open net that performs a self loop and in every cycle produces a token in an output place, thereby violating any bound.

In the rest of this section, we give a trace-based semantics for open nets based on which we prove $b$-accordance to be a precongruence with regard to the open net composition operator $\oplus$.

Our trace-based semantics for $b$-boundedness of an open net $N$ is part of the $b$-bounded stopdead-semantics [3132. A bound violation is a marking that is not $b$-bounded, and we investigate the traces leading to such a bound violation, called strict bound $d_{b}$-violators. A bound violation is regarded as catastrophic because it cannot be corrected. Thus, the behavior after a bound violation does not matter, and we will hide all possible differences by treating all strict bound $_{b}$-violators and their continuations in the same way. Technically, we achieve the hiding by including all continuations of strict bound $b_{b}$-violators in a set bound $d_{b}$, the set of bound $_{b}$-violators. For the same reason, bound $b$ is contained in the second component of our b-bounded semantics, the language of $N$. This technique is called flooding in [14.

Definition 5.2 (b-bounded semantics). Let $N$ be a labeled net. A trace $w$ is a strict bound $d_{b}$-violator of $N$ if there exists a marking $m$ with $m_{N} \stackrel{w}{\Longrightarrow} m$ that is not b-bounded; a continuation of a strict bound $b_{b}$-violator of $N$ is a bound $b_{b}$ violator of $N$. The b-bounded semantics of $N$ is defined by

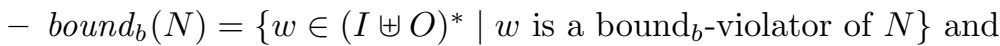

- $L_{b}(N)=L(N) \cup$ bound $_{b}(N)$.

We recall properties of bound $d_{b}$ and $L_{b}$, and how the bound $b^{-}$and $L_{b}$-semantics of a composition is calculated. We also consider a labeled net $N / A, A \subseteq \Sigma$ and we use $\phi(w)$ to denote $\left.w\right|_{\Sigma \backslash A}$. We canonically extend the notion of $\phi(w)$ pointwise to sets of traces.

Proposition 5.3. For two composable labeled nets $N_{1}$ and $N_{2}$, we have

$$
\begin{aligned}
& \text { 1. } \text { bound }_{b}\left(N_{1} \| N_{2}\right)=\uparrow\left(\left(\text { bound }_{b}\left(N_{1}\right) \| L_{b}\left(N_{2}\right)\right) \cup\left(L_{b}\left(N_{1}\right) \| \text { bound }_{b}\left(N_{2}\right)\right)\right) \\
& \text { 2. } \text { bound }_{b}\left(N_{1} \Uparrow N_{2}\right)=\uparrow\left(\left(\text { bound }_{b}\left(N_{1}\right) \Uparrow L_{b}\left(N_{2}\right)\right) \cup\left(L_{b}\left(N_{1}\right) \Uparrow \text { bound }_{b}\left(N_{2}\right)\right)\right) \text {, }
\end{aligned}
$$


3. $L_{b}\left(N_{1} \| N_{2}\right)=\left(L_{b}\left(N_{1}\right) \| L_{b}\left(N_{2}\right)\right) \cup \operatorname{bound}_{b}\left(N_{1} \| N_{2}\right)$,

4. $L_{b}\left(N_{1} \Uparrow N_{2}\right)=\left(L_{b}\left(N_{1}\right) \Uparrow L_{b}\left(N_{2}\right)\right) \cup \operatorname{bound}_{b}\left(N_{1} \Uparrow N_{2}\right)$,

5. $\operatorname{bound}_{b}(N / A)=\left\{\phi(w) \mid w \in\right.$ bound $\left._{b}(N)\right\}$,

6. $L_{b}(N / A)=\left\{\phi(w) \mid w \in L_{b}(N)\right\}$,

and for two composable open nets $N_{1}$ and $N_{2}$, we have

7. bound $_{b}\left(N_{1} \oplus N_{2}\right)=\uparrow\left(\left(\right.\right.$ bound $\left._{b}\left(N_{1}\right) \Uparrow L_{b}\left(N_{2}\right)\right) \cup\left(L_{b}\left(N_{1}\right) \Uparrow\right.$ bound $\left.\left._{b}\left(N_{2}\right)\right)\right)$

8. $L_{b}\left(N_{1} \oplus N_{2}\right)=\left(L_{b}\left(N_{1}\right) \Uparrow L_{b}\left(N_{2}\right)\right) \cup \operatorname{bound}_{b}\left(N_{1} \oplus N_{2}\right)$.

Proof. (1) has already been proved for $b=1$ in [33, Theorem 3.3.3]; we can use the same considerations to show that this result can be generalized to an arbitrary bound $b \in \mathbb{N}_{+} ;(2)$ follows directly from (1) by Definition 2.7.

(3)

$$
\begin{aligned}
& L_{b}\left(N_{1} \| N_{2}\right) \\
= & \left(L\left(N_{1}\right) \cup \text { bound }_{b}\left(N_{1}\right)\right) \|\left(L\left(N_{2}\right) \cup \text { bound }_{b}\left(N_{2}\right)\right) \cup \text { bound }_{b}\left(N_{1} \| N_{2}\right) \\
= & \left(L\left(N_{1}\right) \| L\left(N_{2}\right)\right) \cup\left(\text { bound }_{b}\left(N_{1}\right) \| L\left(N_{2}\right)\right) \cup\left(\text { bound }_{b}\left(N_{2}\right) \| L\left(N_{1}\right)\right) \\
\cup & \left(\text { bound }_{b}\left(N_{1}\right) \| \text { bound }_{b}\left(N_{2}\right) \cup \text { bound }_{b}\left(N_{1} \| N_{2}\right)\right. \\
& \left\{\text { By } L\left(N_{2}\right) \subseteq L_{b}\left(N_{2}\right), L\left(N_{1}\right) \subseteq L_{b}\left(N_{1}\right), \text { bound }_{b}\left(N_{1}\right) \subseteq L_{b}\left(N_{1}\right),(1)\right\} \\
= & L\left(N_{1}\right) \| L\left(N_{2}\right) \cup \text { bound }_{b}\left(N_{1} \| N_{2}\right) \\
& \{\text { by }[33, \text { Theorem } 3.1 .7(4)]\} \\
= & L\left(N_{1} \| N_{2}\right) \cup \text { bound }_{b}\left(N_{1} \| N_{2}\right)
\end{aligned}
$$

(4) follows the same argumentation as (3); (5) and (6) are obvious; and (7) and (8) have already been proved in [32, Theorem 30].

If we consider the composition of two open nets $N_{1}$ and $N_{2}$, then its $b$ bounded semantics coincides with that of the parallel composition of the two environments env $\left(N_{1}\right) \Uparrow \operatorname{env}\left(N_{2}\right)$.

Lemma 5.4. For two composable open nets $N_{1}$ and $N_{2}$, we have

1. bound $_{b}\left(N_{1} \oplus N_{2}\right)=$ bound $_{b}\left(\operatorname{env}\left(N_{1}\right) \Uparrow \operatorname{env}\left(N_{2}\right)\right)$, and

2. $L_{b}\left(N_{1} \oplus N_{2}\right)=L_{b}\left(\operatorname{env}\left(N_{1}\right) \Uparrow \operatorname{env}\left(N_{2}\right)\right)$.

Proof. The first item follows by Proposition 5.3(2) and (7). For the second item, we have $L_{b}\left(N_{1} \oplus N_{2}\right)=L\left(N_{1} \oplus N_{2}\right) \cup$ bound $\left(N_{1} \oplus N_{2}\right)$ by Definition 5.2 . With $L\left(N_{1} \oplus N_{2}\right)=L\left(\operatorname{env}\left(N_{1}\right) \Uparrow e n v\left(N_{2}\right)\right)$ by [32, Theorem 18(1)] and the first item, we conclude with Definition 5.2 that $L_{b}\left(N_{1} \oplus N_{2}\right)=L_{b}\left(\operatorname{env}\left(N_{1}\right) \Uparrow \operatorname{env}\left(N_{2}\right)\right)$.

The presence of bound $d_{b}$-violators of $N_{1}$ and $N_{2}$ is closely related to the question whether $N_{1}$ and $N_{2}$ are $b$-controller of each other. This result directly follows from Proposition $5.3(7)$.

Corollary 5.5 (b-boundedness vs. $b$-bounded semantics). For two composable open nets $N_{1}$ and $N_{2}$ such that $N_{1} \oplus N_{2}$ is a closed net, we have

$$
\begin{aligned}
N_{1} \text { is a b-controller of } N_{2} \text { iff } \begin{array}{l}
L_{b}\left(N_{1}\right) \cap \text { bound } \\
\text { bound }\left(N_{2}\right)
\end{array}\left(N_{1}\right) \cap L_{b}\left(N_{2}\right)=\emptyset .
\end{aligned}
$$


The inclusion of the $b$-bounded semantics of open nets defines a refinement relation. With the next theorem, we prove that it coincides with $b$-accordance; in other words, we provide a trace-based characterization for the latter.

Theorem 5.6 ( $b$-accordance and $b$-bounded semantics inclusion coincide). For two interface-equivalent open nets Impl and Spec, we have

$$
\begin{array}{cl}
\text { Impl } \sqsubseteq_{b, a c c} \text { Spec } \quad \text { iff } \quad & \text { bound }_{b}(\operatorname{Impl}) \subseteq \text { bound }_{b}(\text { Spec }) \text { and } \\
& L_{b}(\text { Impl }) \subseteq L_{b}(\text { Spec }) .
\end{array}
$$

Proof. $\Rightarrow$ : Use the proof of [32, Theorem 33].

$\Leftarrow$ : Proof by contraposition. Consider an open net $C$ such that $\operatorname{Impl} \oplus C$ and Spec $\oplus C$ are closed nets. Assume that $C$ is not a $b$-controller of $I m p l$. Then we have $L_{b}(\operatorname{Impl}) \cap$ bound $_{b}(C) \neq \emptyset$ or bound $(\operatorname{Impl}) \cap L_{b}(C) \neq \emptyset$ by Corollary 5.5 . Because of the assumed inclusions, we also have $L_{b}($ Spec $) \cap$ bound $_{b}(C) \neq \emptyset$ or bound $_{b}($ Spec $) \cap L_{b}(C) \neq \emptyset$. Again with Corollary 5.5. we see that $C$ is not a $b$-controller of Spec.

We show that $b$-accordance is a precongruence for the composition operators $\|$ and $\oplus$.

Proposition 5.7. Inclusion of bound $d_{b}$-and $L_{b}$-traces is a precongruence for $\|$ and hiding on labeled nets.

Proof. Follows from Proposition 5.3(1),(3),(5),(6).

Theorem 5.8 ( $b$-accordance is a precongruence). The $b$-accordance relation $\sqsubseteq_{b, a c c}$ is a precongruence w.r.t. the composition operator $\oplus$.

Proof. Let Impl and $S p e c$ be interface-equivalent open nets such that $\operatorname{Impl} \sqsubseteq_{b, a c c}$ Spec, and let $C$ be an open net that is composable with Impl (and Spec by interface equivalence). Then:

$$
\begin{aligned}
& \text { bound }_{b}(\operatorname{Impl} \oplus C)=\{\text { by Proposition 5.3. (7) }\} \\
& \uparrow\left(\left(\text { bound }_{b}(\operatorname{Impl}) \Uparrow L_{b}(C)\right) \cup\left(L_{b}(\operatorname{Impl}) \Uparrow \text { bound }_{b}(C)\right)\right) \\
& \subseteq\{\text { by Theorem 5.6 and Definition 2.7 }\} \\
& \uparrow\left(\left(\text { bound }_{b}(\text { Spec }) \Uparrow L_{b}(C)\right) \cup\left(L_{b}(\text { Spec }) \Uparrow \text { bound }_{b}(C)\right)\right) \\
& =\text { bound }_{b}(\text { Spec } \oplus C) \text { by Proposition 5.3(7) } \\
& L_{b}(\operatorname{Impl} \oplus C)=\{\text { by Proposition 5.3(8) }\} \\
& \left(L_{b}(\operatorname{Impl}) \Uparrow L_{b}(C)\right) \cup \text { bound }_{b}(\operatorname{Impl} \oplus C) \\
& \subseteq\{\text { by Theorem 5.6 and Definition 2.7 }\} \\
& \left(L_{b}(\text { Spec }) \Uparrow L_{b}(C)\right) \cup \text { bound }_{b}(\operatorname{Impl} \oplus C) \\
& \subseteq\left\{\text { by bound }(\operatorname{Impl} \oplus C) \subseteq \text { bound }_{b}(\operatorname{Spec} \oplus C)\right\} \\
& \left(L_{b}(\text { Spec }) \Uparrow L_{b}(C)\right) \cup \text { bound }_{b}(\text { Spec } \oplus C) \\
& =L_{b}(\text { Spec } \oplus C) \text { by Proposition 5.3 (8). }
\end{aligned}
$$

Thus, Impl $\oplus C \sqsubseteq_{b, a c c}$ Spec $\oplus C$ by Theorem 5.6 . 


\section{Bounded nets and no final markings}

In this section, we require the composition of open nets to be bounded and responsive; that is, we ignore final markings as in Sect. 3. The resulting variant of responsiveness, bounded responsiveness or $b$-responsiveness for short, is similar to safe $P$-deadlock equivalence in 33 .

Definition 6.1 (b-responsiveness). Let $N_{1}$ and $N_{2}$ be composable open nets. A marking of $N_{1} \oplus N_{2}$ is $b$-responsive if it is responsive and $b$-bounded. The open nets $N_{1}$ and $N_{2}$ are $b$-responsive if their composition $N_{1} \oplus N_{2}$ is a closed net and every reachable marking in $N_{1} \oplus N_{2}$ is b-responsive.

Two open nets are $b$-responsive if at least one net can repeatedly talk while respecting the message bound $b$. In fact, we can prove that, due to $b$-responsiveness, each net always has the chance to send a message (possibly after some messages from the other net). Thus, the word 'responsive' is really justified here.

Proposition 6.2. Let open nets $N_{1}$ and $N_{2}$ be b-responsive. Then, from any reachable marking $m$ of $N_{1} \oplus N_{2}$, markings $m_{1}$ and $m_{2}$ are reachable such that $m_{1} \stackrel{t_{1}}{\rightarrow}$ and $m_{2} \stackrel{t_{2}}{\rightarrow}$ with $t_{1}^{\bullet} \cap O_{1} \neq \emptyset$ and $t_{2}^{\bullet} \cap O_{2} \neq \emptyset$.

Proof. Proof by contradiction. Assume that there exists a marking $m$ from which no suitable $m_{1}$ or $m_{2}$ is reachable. This contradicts Definition 6.1. because all markings in $N_{1} \oplus N_{2}$ are $b$-responsive and thus responsive.

Now assume that there exists an $m$ from which only a marking $m_{1}$ is reachable but no $m_{2}$. Then, in $N_{1} \oplus N_{2}$ there exists a run to a marking $m_{1}$ enabling some $t_{1}$. No tokens are put onto $I_{1}=O_{2}$ in this run; otherwise, we would have found an $m_{2}$ just before such a firing. Hence, no transitions of $N_{2}$ are needed to enable $t_{1}$, and we can assume that all transitions of the run belong to $N_{1}$. Consequently, no token is removed from $O_{1}=I_{2}$. Now we fire $t_{1}$ and reach some $m^{\prime}$ with at least one token more on $O_{1}$. If $m^{\prime}$ has an $m_{2}^{\prime}$ as claimed in the lemma, this can also serve for $m$ as $m_{2}$. Hence, $m_{2}^{\prime}$ does not exist, but some $m_{1}^{\prime}$ must, as argued previously. We repeat this argument, and each time the token count on $O_{1}$ increases until bound $b$ is violated. However, this contradicts Definition 6.1, stating that $N_{1} \oplus N_{2}$ is $b$-bounded. As a consequence, a marking $m_{2}$ must be reachable from $m$.

We redefine the notion of a controller and of accordance for this variant of responsiveness to $b r$-controller and $b r$-accordance. As $b r$-accordance shall turn out not to be a precongruence, we also introduce its coarsest precongruence.

Definition 6.3 ( $b r$-controller, $b r$-accordance). An open net $C$ is a $b r$-controller of an open net $N$ if $N$ and $C$ are $b$-responsive.

For interface-equivalent open nets Impl and Spec, Impl br-accords with Spec, denoted by $\operatorname{Impl} \sqsubseteq_{b r, a c c} S p e c$, if for all open nets $C$ holds: $C$ is a $b r$-controller of Spec implies $C$ is a $b r$-controller of $I m p l$.

We denote the coarsest precongruence contained in $\sqsubseteq_{b r, a c c}$ by $\sqsubseteq_{b r, a c c}^{c}$. 


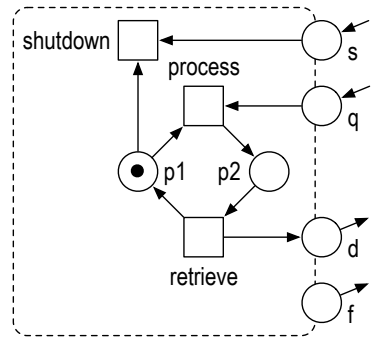

(a) Open net $D$

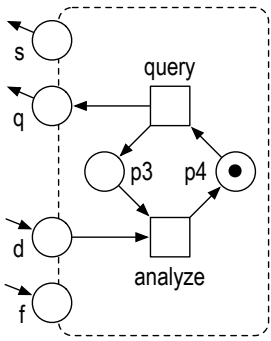

(b) Open net $U$

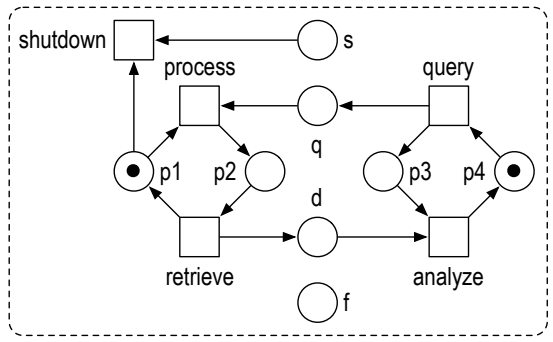

(c) Open net $D \oplus U$

Fig. 8: Open nets modeling a database server, a user, and their composition. In addition to the models, we have $\Omega_{D}=\{[]\}, \Omega_{U}=\left\{\left[p_{4}\right]\right\}$ and $\Omega_{D \oplus U}=\left\{\left[p_{4}\right]\right\}$.

Example 6.1. Figure 8 shows three open systems, each modeled as an open net. The open net $D$ models a database server. After processing a query (input place $q$ ), it responds with the retrieved data (output place $d$ ). A user may shut down $D$ by sending a shutdown message (input place $s$ ). $D$ has the (unused) capability to forward messages (output place $f$ ). The open net $U$ models a user of the database. It repeatedly queries the database and analyzes the returned data. $U$ never sends a shutdown message and ignores any forwarded message from $D$. The open nets $D$ and $U$ are composable. Their composition $D \oplus U$ is a 1-bounded closed net, which is depicted in Fig. 8c.

Figure 9 depicts a modified database server $D^{\prime}$. It has the same functionality as $D$ but forwards a shutdown message to the output place $f$. No $b r$-controller of $D$ sends a message $s$, as otherwise $D$ could fire shutdown and then could not produce any output, contradicting Proposition 6.2. Thus, $D^{\prime}$ br-accords with $D$. Vice versa, no $b r$-controller of $D^{\prime}$ sends a message $s$ because after sending $f, D^{\prime}$ cannot produce any token on $d$ or $f$. Thus, also $D$ br-accords with $D^{\prime}$. Observe that these two statements hold for any bound $b$.

Extending the example with the open nets $X$ and $Y$ in Fig. 10 , we can show that $b r$-accordance is not compositional: $X$ is a $b r$-controller of $D^{\prime} \oplus Y$ but not of $D \oplus Y$. Whereas the transition activate of $Y$ can be fired in $\left(D^{\prime} \oplus Y\right) \oplus X$ (enabling $b r$-responsiveness), it cannot be fired in $(D \oplus Y) \oplus X$. 


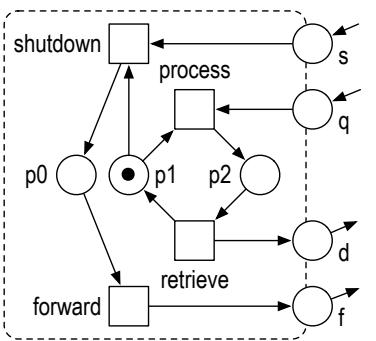

Fig. 9: The open net $D^{\prime}$ modeling a modified database server. We have $\Omega_{D^{\prime}}=$ $\left\{\left[p_{0}\right]\right\}$.

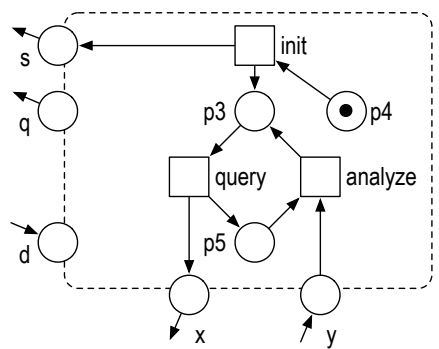

(a) Open net $X$

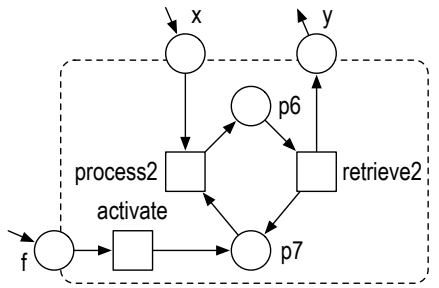

(b) Open net $Y$

Fig. 10: Two open nets proving that $b r$-accordance (and $b f r$-accordance, see Sect. 7) is not a precongruence with regard to open net composition $\oplus$. In addition to the models, we have $\Omega_{X}=\left\{\left[p_{3}\right]\right\}$ and $\Omega_{Y}=\left\{\left[p_{7}\right]\right\}$.

As in Sect. 4, we give a trace-based semantics for $b$-responsiveness. Then, we characterize the coarsest precongruence that is contained in the $b r$-accordance relation.

\subsection{A trace-based semantics for bounded responsiveness}

Our trace-based semantics for $b$-responsiveness of an open net $N$ essentially combines the stop-semantics of Definition 3.4 and the $b$-bounded semantics of Definition 5.2. It consists of the set of all bound $_{b}$-violators, the flooded language, and the flooded stop-semantics.

Definition 6.4 (b-bounded stop-semantics). The b-bounded stop-semantics of a labeled net $N$ is defined by the sets of traces

- $\operatorname{bound}_{b}(N)$

- $L_{b}(N)$, and

$-\operatorname{stop}_{b}(N)=\operatorname{stop}(N) \cup$ bound $_{b}(N)$.

The following lemma implies that, for the purpose of characterizing brcontrollers in Proposition 6.6 below, we could also work with a variant of the 
$b$-bounded stop-semantics that contains stop-traces instead of $s$ sto $_{b}$-traces. This observation makes the proof of Proposition 6.6 easy. Still, we use the $s_{0} p_{b}$-traces in this semantics because it gives a better sufficient condition for $b r$-accordance in Theorem 6.7 .

Lemma 6.5. For composable open nets $N_{1}$ and $N_{2}$ with $L_{b}\left(N_{1}\right) \cap \operatorname{bound}_{b}\left(N_{2}\right)=$ $\emptyset$ and bound $\left(N_{1}\right) \cap L_{b}\left(N_{2}\right)=\emptyset$, we have

$$
\operatorname{stop}\left(N_{1}\right) \cap \operatorname{stop}\left(N_{2}\right)=\emptyset \text { iff } \operatorname{stop}_{b}\left(N_{1}\right) \cap \operatorname{stop}_{b}\left(N_{2}\right)=\emptyset \text {. }
$$

Proof. $\Leftarrow$ : Follows immediately from Definition 6.4.

$\Rightarrow$ : We can write stop $_{b}\left(N_{1}\right) \cap$ stop $_{b}\left(N_{2}\right)$ as a union of four intersections

$$
\begin{aligned}
\operatorname{stop}_{b}\left(N_{1}\right) \cap \operatorname{stop}_{b}\left(N_{2}\right)= & \left(\operatorname{stop}\left(N_{1}\right) \cap \text { bound }_{b}\left(N_{2}\right)\right) \\
& \cup\left(\operatorname{bound}_{b}\left(N_{1}\right) \cap \operatorname{stop}\left(N_{2}\right)\right) \\
& \cup\left(\operatorname{bound}{ }_{b}\left(N_{1}\right) \cap \operatorname{bound}{ }_{b}\left(N_{2}\right)\right) \\
& \cup\left(\operatorname{stop}\left(N_{1}\right) \cap \operatorname{stop}\left(N_{2}\right)\right),
\end{aligned}
$$

all of which are empty:

$-\operatorname{stop}\left(N_{1}\right) \cap \operatorname{bound}_{b}\left(N_{2}\right)=\emptyset$, by $L_{b}\left(N_{1}\right) \cap \operatorname{bound}_{b}\left(N_{2}\right)=\emptyset$ and $\operatorname{stop}\left(N_{1}\right) \subseteq$ $L\left(N_{1}\right) \subseteq L_{b}\left(N_{1}\right)$

- $\operatorname{bound}_{b}\left(N_{1}\right) \cap \operatorname{stop}\left(N_{2}\right)=\emptyset$, by bound $\left(N_{1}\right) \cap L_{b}\left(N_{2}\right)=\emptyset$ and $\operatorname{stop}\left(N_{2}\right) \subseteq$ $L\left(N_{2}\right) \subseteq L_{b}\left(N_{2}\right)$

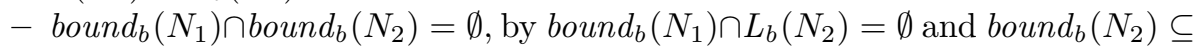
$L_{b}\left(N_{2}\right)$

$-\operatorname{stop}\left(N_{1}\right) \cap \operatorname{stop}\left(N_{2}\right)=\emptyset$ by assumption.

Some open net $C$ is a $b r$-controller if and only if it is a $b$-controller and an $r$-controller. Thus, Corollary 5.5 and Proposition 3.7 in combination with Lemma 6.5 give the following characterization of $b$-responsiveness.

Proposition 6.6 ( $b$-responsiveness vs. $b$-bounded stop-semantics). For composable open nets $N_{1}$ and $N_{2}$ such that $N_{1} \oplus N_{2}$ is a closed net, we have

$$
\begin{aligned}
& \operatorname{stop}_{b}\left(N_{1}\right) \cap \operatorname{stop}_{b}\left(N_{2}\right)=\emptyset \text { and } \\
& N_{1} \text { and } N_{2} \text { are b-responsive iff } L_{b}\left(N_{1}\right) \cap \text { bound } d_{b}\left(N_{2}\right)=\emptyset \text { and } \\
& \text { bound }_{b}\left(N_{1}\right) \cap L_{b}\left(N_{2}\right)=\emptyset \text {. }
\end{aligned}
$$

Inclusion of the $b$-bounded stop-semantics defines a refinement relation which implies $b r$-accordance.

Theorem 6.7 (b-bounded stop-inclusion implies $b r$-accordance). For two interface-equivalent open nets Impl and Spec, we have

bound $_{b}(\operatorname{Impl}) \subseteq$ bound $_{b}($ Spec $)$ and $L_{b}($ Impl $) \subseteq L_{b}($ Spec $)$ and implies $\quad$ Impl $\sqsubseteq_{b r, a c c}$ Spec. $\operatorname{stop}_{b}(\operatorname{Impl}) \subseteq \operatorname{stop}_{b}($ Spec $)$ 
Proof. Proof by contraposition. Consider an open net $C$ such that $\operatorname{Impl} \oplus C$ and Spec $\oplus C$ are closed nets. Assume that $C$ is not a $b r$-controller of Impl. Then, $I m p l$ and $C$ are not $b$-responsive by Definition 6.3, and we have $\operatorname{stop}_{b}(\operatorname{Impl}) \cap$ stop $_{b}(C) \neq \emptyset, L_{b}(\operatorname{Impl}) \cap$ bound $_{b}(C) \neq \emptyset$, or bound $(\operatorname{Impl}) \cap L_{b}(C) \neq \emptyset$ by Proposition 6.6. Because of the assumed inclusions, we have $\operatorname{stop}_{b}($ Spec $) \cap \operatorname{stop}_{b}(C) \neq \emptyset$, $L_{b}($ Spec $) \cap$ bound $_{b}(C) \neq \emptyset$, or bound $($ Spec $) \cap L_{b}(C) \neq \emptyset$. Again with Proposition 6.6. we see that $S p e c$ and $C$ are not $b$-responsive; that is, $C$ is not a $b r$ controller of Spec.

The converse of Theorem 6.7 does not hold in general, as the next example shows.

Example 6.2. Recall that the open net $D^{\prime} b r$-accords with the open net $D$ for every $b \in \mathbb{N}_{+}$(see Example 6.1) although the language of $D^{\prime}$ is not contained in the language of $D$. For instance, we have $s f \in L_{b}\left(D^{\prime}\right) \backslash L_{b}(D)$. However, sf cannot be used reliably by any $b r$-controller of $D$.

The cause of the counterexample in Example 6.2 and, thus, the reason why the converse of Theorem 6.7 does not hold is that $b r$-accordance ignores those parts of open nets Impl and Spec that cannot be used reliably - that is, those markings that cannot be covered in the composition with any br-controller. In contrast, standard trace-based semantics compare the two open nets as a whole.

That standard language inclusion can be too strict has been observed for a stronger criterion than $b$-responsiveness in 21|5|23. Mooij et al. 23] propose two solutions to overcome this problem. The first idea is to restrict the class of open nets considered to those where every place and transition can be covered. The second idea is to encode the covering nature of $b r$-accordance in the trace-based semantics. In the following, we work out the latter idea in the present setting.

\subsection{A coverable trace-based semantics for $b$-responsiveness}

We aim to encode the covering nature of $b r$-accordance in the $b$-bounded stopsemantics. To achieve this, we introduce a set that captures all br-uncoverable traces; that is, traces $w$ that cannot be executed by (the environment of) any $b r$-controller of $N$, regardless whether $w$ can be executed in env $(N)$ or not.

Replacing in every trace set of the $b$-bounded stop-semantics of an open net $N$ the set of bound $_{b}$-violators by the set of $b r$-uncoverable traces yields the coverable b-bounded stop-semantics of $N$. This semantics differs from the previous trace semantics, as the $b r$-uncoverable traces are an external characterizationthey quantify over all $b r$-controllers of $N$. The latter does not cause a problem, because we can calculate this set.

Definition 6.8 (coverable $b$-bounded stop-semantics). Let $N$ be an open net. A word $w \in(I \uplus O)^{*}$ is a br-uncoverable trace of $N$ if there does not exist a br-controller $C$ of $N$ with $w \in L_{b}(C)$. The coverable b-bounded stop-semantics of $N$ is defined by the three sets of traces

- $\operatorname{uncov}_{b r}(N)=\left\{w \in(I \uplus O)^{*} \mid w\right.$ is an br-uncoverable trace of $\left.N\right\}$, 
- $u L_{b r}(N)=L(N) \cup$ uncov $_{b r}(N)$, and

- ustop br $(N)=\operatorname{stop}(N) \cup$ uncov br $(N)$.

Example 6.3. As mentioned in Example 6.1, for any bound $b$, there exists no $b r$-controller of $D$ or $D^{\prime}$ that marks the place $s$. Thus, $s \in$ uncov $_{b r}(D)$ and $s \in$ uncovbr $_{b}\left(D^{\prime}\right)$, for instance.

By Proposition 6.6. a bound $b_{b}$-violator of an open net is an $b r$-uncoverable trace of $N$. So we directly conclude that the set of bound $d_{b}$-violators of $N$ is contained in the set of $b r$-uncoverable traces of $N$. As a result, the coverable $b$ bounded stop-semantics extends the $b$-bounded stop-semantics by flooding more traces: bound $_{b}$-violators and $b r$-uncoverable traces.

Lemma 6.9 (b-bounded stop-semantics is included). For any open net $N$, we have

- $\operatorname{bound}_{b}(N) \subseteq$ uncov br $(N)$,

$-L_{b}(N) \subseteq u L_{b r}(N)$, and

$-\operatorname{stop}_{b}(N) \subseteq$ ustop $_{b r}(N)$.

Inclusion of the flooded stop-traces, the flooded language, and the $b r$-uncoverable traces defines a refinement relation. We show that an open net Impl braccords with an open net Spec if the respective traces of Impl's coverable $b$ bounded stop-semantics are included in the respective traces of Spec's coverable $b$-bounded stop-semantics. For the proof, we use the following two lemmata. The first lemma states that for every trace $w$, which is neither a trace nor a $b r$-uncoverable trace of $N$, there exists a $b r$-controller of $N$ containing $w$ in its

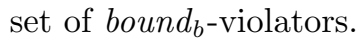

Lemma 6.10. Let $N$ be an open net. If $w \notin u L_{b r}(N)$, then there exists a brcontroller $C$ of $N$ with $w \in$ bound $_{b}(C)$.

Proof. Let $w \notin u L_{b r}(N)$ with $w=w_{1} \ldots w_{k}$ for $j=1, \ldots, k, w_{j} \in I_{N} \uplus O_{N}$. As $w \notin$ uncov $_{b r}(N)$, there exists a $b r$-controller $C$ of $N$ with $w \in L_{b}(C)$ by Definition 6.8. If $w \notin$ bound $_{b}(C)$, we construct from $w$ and $C$ a $b r$-controller $N_{w}^{\text {bound }} \oplus C^{\prime}$ of $N$ with bound $b_{b}$-violator $w$ as follows: In a first step, we construct an open net $N_{w}$ that basically shifts all tokens from $N$ to $C$, and vice versa. Moreover, $N_{w}$ tracks whether a firing sequence in $C$ is a prefix of $w$, and subsequently moving a token in $N_{w}$ from an initially marked place $p_{0}$ to a place $p_{k}$. Intuitively, a token on a place $p_{j}$ means we already encountered the trace $w_{1} \ldots w_{j}$. For shifting, we introduce several interface transitions in $N_{w}$ for each interface place in $N$. In a second step, if and only if the place $p_{k}$ is markedthat is, we encountered the trace $w_{1} \ldots w_{k}=w$-a "disaster" transition $t_{\text {disaster }}$ will be enabled, which may produce an unlimited number of tokens onto an inner place $p_{\text {disaster }}$. The latter construction yields the open net $N_{w}^{\text {bound }}$. This construction guarantees that $w$ is a bound $b_{b}$-violator of $N_{w}^{\text {bound }} \oplus C^{\prime}$.

Let $I^{\prime}=\left\{i^{\prime} \mid i \in I_{C}\right\}$ and $O^{\prime}=\left\{o^{\prime} \mid o \in O_{C}\right\}$ be "fresh" copies of $I_{C}$ and $O_{C}$. We derive the open net $C^{\prime}=\left(P_{C}, T_{C}, F_{C}^{\prime}, m_{C}, I^{\prime}, O^{\prime}, \emptyset\right)$ from $C$ by renaming the interface of $C$ and adjusting the flow relation accordingly. We define the open net $N_{w}=\left(P^{\prime}, T^{\prime}, F^{\prime}, m_{N_{w}}, O_{N} \uplus O_{C^{\prime}}, I_{N} \uplus I_{C^{\prime}}, \emptyset\right)$ with 


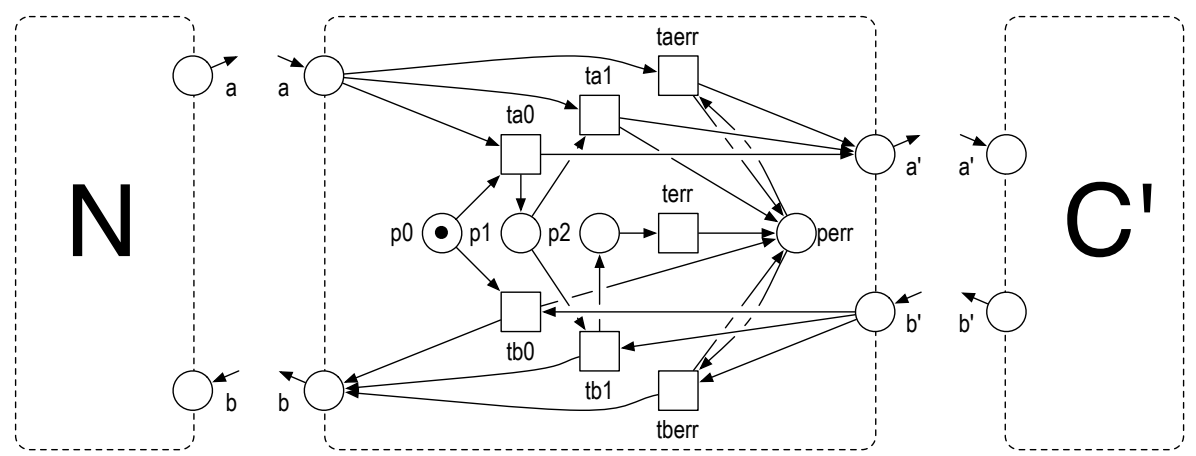

Fig. 11: Illustration of the construction of the open net $N_{w}$ for $N$ with $O_{N}=\{a\}$, $I_{N}=\{b\}$, and $w=a b$.

$$
\begin{aligned}
-P^{\prime}= & \left\{p_{i} \mid 0 \leq i \leq k\right\} \\
& \uplus\left\{p_{\text {err }}\right\}, \\
-T^{\prime}= & \left\{t_{i}^{x} \mid 0 \leq i \leq k-1 \wedge x \in O_{N} \uplus I_{N}\right\} \\
& \left.\uplus t_{e r r}^{x} \mid x \in O_{N} \uplus I_{N}\right\} \\
& \uplus\left\{t_{\text {err }}\right\}, \\
& \left\{\left(x, t_{i}^{x}\right),\left(t_{i}^{x}, x^{\prime}\right),\left(p_{i}, t_{i}^{x}\right) \mid 0 \leq i \leq k-1 \wedge x \in O_{N}\right\} \\
-F^{\prime}= & \left.\uplus\left(x^{\prime}, t_{i}^{x}\right),\left(t_{i}^{x}, x\right),\left(p_{i}, t_{i}^{x}\right) \mid 0 \leq i \leq k-1 \wedge x \in I_{N}\right\} \\
& \uplus\left\{\left(t_{i}^{x}, p_{i+1}\right) \mid 0 \leq i \leq k-1 \wedge x \in O_{N} \uplus I_{N} \wedge x=w_{i+1}\right\} \\
& \uplus\left\{\left(t_{i}^{x}, p_{\text {err }}\right) \mid 0 \leq i \leq k-1 \wedge x \in O_{N} \uplus I_{N} \wedge x \neq w_{i+1}\right\} \\
& \uplus\left\{\left(x, t_{\text {err }}^{x}\right),\left(t_{\text {err }}^{x}, x^{\prime}\right),\left(p_{\text {err }}, t_{\text {err }}^{x}\right),\left(t_{\text {err }}^{x}, p_{\text {err }}\right) \mid x \in O_{N}\right\} \\
& \uplus\left\{\left(x^{\prime}, t_{\text {err }}^{x}\right),\left(t_{\text {err }}^{x}, x\right),\left(p_{\text {err }}, t_{\text {err }}^{x}\right),\left(t_{\text {err }}^{x}, p_{\text {err }}\right) \mid x \in I_{N}\right\} \\
& \uplus\left\{\left(p_{k}, t_{\text {err }}\right),\left(t_{\text {err }}, p_{\text {err }}\right)\right\}, \\
-m_{N_{w}}= & {\left[p_{0}\right] . }
\end{aligned}
$$

Figure 11 illustrates the construction of $N_{w}$. Clearly, we have $L(C)=L\left(N_{w} \oplus\right.$ $\left.C^{\prime}\right)$, bound $(C)=$ bound $_{b}\left(N_{w} \oplus C^{\prime}\right)$, and stop $(C)=\operatorname{stop}\left(N_{w} \oplus C^{\prime}\right)$. Therefore, the open net $N_{w} \oplus C^{\prime}$ is a $b r$-controller of $N$ by Proposition 6.6 .

The places $p_{0}, \ldots, p_{k}, p_{\text {err }}$ together always carry one token, and $p_{k}$ gets marked if and only if we encountered a trace whose prefix is $w$. Next, we extend $N_{w}$ to an open net $N_{w}^{\text {bound }}$ by adding a disaster transition $t_{\text {disaster }}$. The transition $t_{\text {disaster }}$ may produce an unlimited number of tokens onto an inner place $p_{\text {disaster }}$ of $N_{w}^{\text {bound }}$. Formally, we define the open net $N_{w}^{\text {bound }}=$ $\left(P^{\prime} \uplus\left\{p_{\text {disaster }}\right\}, T^{\prime} \uplus\left\{t_{\text {disaster }}\right\}, F^{\prime} \uplus F^{\prime \prime}, m_{N_{w}}, O_{N} \uplus O_{C^{\prime}}, I_{N} \uplus I_{C^{\prime}}, \emptyset\right)$ with $F^{\prime \prime}=$ $\left\{\left(p_{k}, t_{\text {disaster }}\right),\left(t_{\text {disaster }}, p_{k}\right),\left(t_{\text {disaster }}, p_{\text {disaster }}\right)\right\}$. Thus, $w \in$ bound $_{b}\left(N_{w}^{\text {bound }} \oplus C^{\prime}\right)$ for the $b r$-controller $N_{w}^{\text {bound }} \oplus C^{\prime}$ of $N$.

The second lemma states that for every trace $w$ of $N$, which is neither a stop-trace nor $b r$-uncoverable, there exists a $b r$-controller of $N$ containing $w$ in its set of stop-traces. 
Lemma 6.11. Let $N$ be an open net. If $w \in L(N) \backslash$ ustop $_{b r}(N)$, then there exists a br-controller $C$ of $N$ with $w \in \operatorname{stop}(C)$.

Proof. Let $w \in L(N) \backslash$ ustop $_{b r}(N)$ with $w=w_{1} \ldots w_{k}$ for $j=1, \ldots, k, w_{j} \in$ $I_{N} \uplus O_{N}$. As $w \notin$ uncov $_{b r}(N)$, there exists a $b r$-controller $C$ of $N$ with $w \in L_{b}(C)$ by Definition 6.8. Then $w \in L(C) \backslash$ bound $_{b}(C)$; otherwise, $C$ is not a $b r$-controller of $N$ by Proposition 6.6. As $w$ is no stop-trace of $N$, there exists an output $o \in O_{N}$ such that $m_{e n v(N)} \stackrel{w o}{\Longrightarrow}$. We conclude that $w o \in L(N)$ and $w o \in L(C)$, thus wo is not a bound $_{b}$-violator of $N$ by Proposition 6.6

Like in the proof of Lemma 6.10, we construct a $b r$-controller $N_{w} \oplus C^{\prime}$ of $N$ with stop-trace $w$ : We track whether a firing sequence in $C$ is a prefix of $w$ by composing $C$ with another open net $N_{w}$, and subsequently moving a token in $N_{w}$ from an initially marked place $p_{0}$ to a place $p_{k}$. Later, if and only if the place $p_{k}$ is marked - that is, we encountered the trace $w_{1} \ldots w_{k}=w$ - we prevent any output from $N_{w}$, but allow input to $N_{w}$. This will make $w$ a stop-trace. Once $N_{w}$ receives one input-for example, $o \in O_{N}$ as discussed above - we make $N_{w}$ transparent again.

Let $I^{\prime}=\left\{i^{\prime} \mid i \in I_{C}\right\}$ and $O^{\prime}=\left\{o^{\prime} \mid o \in O_{C}\right\}$ be "fresh" copies of $I_{C}$ and $O_{C}$. We derive the open net $C^{\prime}=\left(P_{C}, T_{C}, F_{C}^{\prime}, m_{C}, I^{\prime}, O^{\prime}, \emptyset\right)$ from $C$ by renaming the interface of $C$ and adjusting the flow relation accordingly. We define the open net $N_{w}=\left(P^{\prime}, T^{\prime}, F^{\prime}, m_{N_{w}}, O_{N} \uplus O_{C^{\prime}}, I_{N} \uplus I_{C^{\prime}}, \emptyset\right)$ with

$$
\begin{aligned}
-P^{\prime}= & \left\{p_{i} \mid 0 \leq i \leq k\right\} \\
& \uplus\left\{p_{\text {err }}\right\}, \\
-T^{\prime}= & \left\{t_{i}^{x} \mid 0 \leq i \leq k \wedge x \in O_{N}\right\} \\
& \uplus\left\{t_{i}^{x} \mid 0 \leq i \leq k-1 \wedge x \in I_{N}\right\} \\
& \uplus\left\{t_{e r r}^{x} \mid x \in O_{N} \uplus I_{N}\right\} \\
& \uplus\left\{t_{e r r}\right\}, \\
& \left\{\left(x, t_{i}^{x}\right),\left(t_{i}^{x}, x^{\prime}\right),\left(p_{i}, t_{i}^{x}\right) \mid 0 \leq i \leq k \wedge x \in O_{N}\right\} \\
-F^{\prime}= & \left.\uplus\left(x^{\prime}, t_{i}^{x}\right),\left(t_{i}^{x}, x\right),\left(p_{i}, t_{i}^{x}\right) \mid 0 \leq i \leq k-1 \wedge x \in I_{N}\right\} \\
& \uplus\left\{\left(t_{i}^{x}, p_{i+1}\right) \mid 0 \leq i \leq k-1 \wedge x \in O_{N} \uplus I_{N} \wedge x=w_{i+1}\right\} \\
& \uplus\left\{\left(t_{i}^{x}, p_{e r r}\right) \mid 0 \leq i \leq k-1 \wedge x \in O_{N} \uplus I_{N} \wedge x \neq w_{i+1}\right\} \\
& \uplus\left\{\left(t_{k}^{x}, p_{e r r}\right) \mid x \in O_{N}\right\} \\
& \uplus\left\{\left(x, t_{e r r}^{x}\right),\left(t_{e r r}^{x}, x^{\prime}\right),\left(p_{e r r}, t_{e r r}^{x}\right),\left(t_{e r r}^{x}, p_{e r r}\right) \mid x \in O_{N}\right\} \\
& \uplus\left\{\left(x^{\prime}, t_{e r r}^{x}\right),\left(t_{e r r}^{x}, x\right),\left(p_{e r r}, t_{e r r}^{x}\right),\left(t_{e r r}^{x}, p_{e r r}\right) \mid x \in I_{N}\right\} \\
& \uplus\left\{\left(p_{k}, t_{e r r}\right),\left(t_{e r r}, p_{e r r}\right)\right\}, \\
= & {\left[p_{0}\right] . }
\end{aligned}
$$

Figure 12 illustrates the construction of $N_{w}$. Clearly, we have $w \in \operatorname{stop}\left(N_{w} \oplus C^{\prime}\right)$, and the open net $N_{w} \oplus C^{\prime}$ is a $b r$-controller of $N$.

Finally, we show that $b r$-accordance coincides with the refinement relation defined by inclusion of the coverable $b$-bounded stop-semantics. 


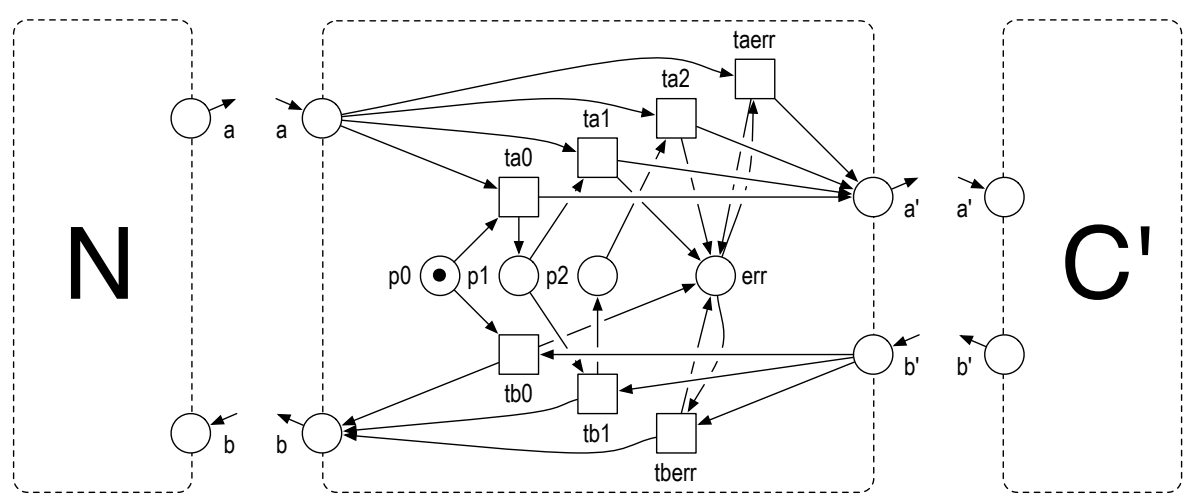

Fig. 12: Illustration of the construction of the open net $N_{w}$ for $N$ with $O_{N}=\{a\}$, $I_{N}=\{b\}$, and $w=a b$.

Theorem 6.12 (coverable $b$-bounded stop-inclusion vs. $b r$-accordance). For two interface-equivalent open nets Impl and Spec, we have

$$
\begin{array}{cl}
\text { Impl } \sqsubseteq_{b r, a c c} \text { Spec } \quad \text { iff } \quad & \text { uncov }_{b r}(\operatorname{Impl}) \subseteq \text { uncov }_{b r}(\text { Spec }), \\
& u L_{b r}(\operatorname{Impl}) \subseteq u L_{b r}(\text { Spec }), \text { and } \\
& \text { ustop }_{b r}(\operatorname{Impl}) \subseteq \text { ustop }_{b r}(\text { Spec }) .
\end{array}
$$

Proof. $\Rightarrow$ : Let $w \notin$ uncov $_{b r}$ (Spec); that is, there exists a $b r$-controller $C$ of Spec with $w \in L_{b}(C)$. Clearly, $C$ is a $b r$-controller of Impl by Impl $\sqsubseteq_{b r, a c c} S p e c$ and, thus, $w \notin$ uncov $_{b r}(\operatorname{Impl})$. This proves uncov $v_{b r}(\operatorname{Impl}) \subseteq$ uncov $_{b r}($ Spec $)$.

Let $w \in u L_{b r}(\operatorname{Impl}) \backslash u n \operatorname{cov}_{b r}(\operatorname{Impl})$ and assume $w \notin u L_{b r}($ Spec $)$. There exists a $b r$-controller $C$ of Spec with $w \in$ bound $_{b}(C)$ by Lemma 6.10. Clearly, $C$ is not a $b r$-controller of Impl by Proposition 6.6, and we have a contradiction to our assumption that Impl $\sqsubseteq_{b r, a c c}$ Spec. Thus, $w \in u L_{b r}($ Spec $)$.

Let $w \in$ ustop $_{b r}(\operatorname{Impl}) \backslash$ uncovbr $_{\text {(Impl }}$ ) and assume $w \notin$ ustop $_{b r}($ Spec $)$. Then, $w \in \operatorname{stop}(\operatorname{Impl}) \subseteq L($ Impl $)$ and $w \in L($ Spec $)$, as $u L_{b r}(\operatorname{Impl}) \subseteq u L_{b r}($ Spec $)$ has been shown already. We can construct a $b r$-controller $C$ of Spec with $w \in \operatorname{stop}(C)$ by Lemma 6.11. Clearly, $C$ is not a $b r$-controller of $I m p l$ by Proposition 6.6. and we have a contradiction to our assumption that Impl $\sqsubseteq_{b r, a c c}$ Spec. Thus, $w \in$ ustop $_{\text {br }}($ Spec $)$.

$\Leftarrow$ : Proof by contraposition. Assume that the three inclusions hold and that $C$ is not a $b r$-controller of $I m p l$. We show that $C$ is not a $b r$-controller of Spec either. If $I m p l$ and $C$ are not $b$-responsive, we have $L_{b}(\operatorname{Impl}) \cap$ bound $d_{b}(C) \neq \emptyset$, or bound $(\operatorname{Impl}) \cap L_{b}(C) \neq \emptyset$, or $\operatorname{stop}_{b}(\operatorname{Impl}) \cap \operatorname{stop}_{b}(C) \neq \emptyset$ by Proposition 6.6. We consider each case separately:

$-w \in L_{b}(\operatorname{Impl}) \cap$ bound $_{b}(C)$ : Then $w \in L_{b}(\operatorname{Impl}) \subseteq u L_{b r}(\operatorname{Impl}) \subseteq u L_{b r}($ Spec $)$ by Lemma 6.9 and assumption. If $w \in L($ Spec $)$ then $C$ is not a $b r$-controller of Spec by $w \in$ bound $_{b}(C)$ and Proposition 6.6 otherwise, if $w \in$ uncov $_{b r}($ Spec $)$, then $C$ is not a $b r$-controller of Spec by $w \in$ bound $_{b}(C) \subseteq L_{b}(C)$ and Definition 4.3 . 
$-w \in$ bound $_{b}(\operatorname{Impl}) \cap L_{b}(C)$ : Then $w \in$ bound $_{b}(\operatorname{Impl}) \subseteq$ uncov $_{b r}(\operatorname{Impl}) \subseteq$ uncov $_{b r}($ Spec $)$ by Lemma 6.9 and assumption. Then $C$ is not a $b r$-controller of $S p e c$ by Definition 4.3 .

$-w \in \operatorname{stop}_{b}(\operatorname{Impl}) \cap \operatorname{stop}_{b}(C):$ Then, $w \in \operatorname{stop}_{b}(\operatorname{Impl}) \subseteq$ ustop $_{b r}(\operatorname{Impl}) \subseteq$ ustop $_{b r}($ Spec $)$ by Lemma 6.9 and assumption. If $w \in \operatorname{stop}($ Spec) then $C$ is not a $b r$-controller of $S p e c$ by $w \in \operatorname{stop}_{b}(C)$ and Proposition 6.6. otherwise, if $w \in$ uncov $_{b r}($ Spec $)$, then $C$ is not a br-controller of $\operatorname{Spec}_{\text {by }} w \in \operatorname{stop}_{b}(C) \subseteq$ $L_{b}(C)$ and Definition 4.3 .

Example 6.4. Example 6.2 shows that $D^{\prime} \sqsubseteq b r, a c c \quad D$ although $s f \in L_{b}\left(D^{\prime}\right) \backslash$ $L_{b}(D)$. This difference is hidden in the coverable $b$-bounded stop-semantics due to flooding: $s f \in u L_{b r}\left(D^{\prime}\right) \subseteq u L_{b r}(D)$.

Despite the external characterization of the trace set uncov $v_{b r}$, we can compute the coverable $b$-bounded stop-semantics of an open net $N$ by using the notion of a most permissive controller [37], which is a controller that can visit all the markings that can be visited using any controller. So, the coverable markings of an open net are the markings that can be visited by a most permissive controller. The construction is not the focus of this article and hence not shown.

As shown in Example6.1, br-accordance is not a precongruence. The next section characterizes the coarsest precongruence that is contained in br-accordance.

\subsection{Deriving the coarsest precongruence for $b$-responsiveness}

To derive the coarsest precongruence for $b$-responsiveness, we need to cope with the restriction to $b$-boundedness and, therefore, add information about bound $b_{b^{-}}$ violators to the $\mathcal{F}^{+}$-semantics in Definition 3.9. For $b r$-accordance, we observed that it does not imply $L_{b}$-inclusion (see Example 6.2, whereas it implies $u L_{b r^{-}}$ inclusion (see Theorem 6.12). Therefore, one could wonder whether we should consider uncoverable traces rather than bound $d_{b}$-violators. The following lemma shows that for the coarsest precongruence the situation is different because this precongruence implies $L_{b}$-inclusion.

Lemma 6.13 ( $\sqsubseteq_{b r, a c c}^{c}$ implies $\left.\sqsubseteq_{b, a c c}\right)$. For interface-equivalent open nets Impl and Spec, we have

$$
\text { Impl } \sqsubseteq_{b r, a c c}^{c} \text { Spec implies Impl } \sqsubseteq_{b, a c c} \text { Spec. }
$$

Proof. Assume $\operatorname{Impl} \nsubseteq_{b, a c c}$ Spec and consider a $b$-controller $A$ of Spec which is not a $b$-controller of Impl. Then the construction in Fig. 13 shows that open net $C$ in Fig. $13 \mathrm{~b}$ is a $b r$-controller of $\operatorname{Spec} \oplus A^{\prime}$ but not of $\operatorname{Impl} \oplus A^{\prime}$. This contradicts that $\operatorname{Impl} \sqsubseteq_{b r, a c c}^{c}$ Spec.

In the light of Lemma 6.13, we add information about bound $d_{b}$-violators to the $\mathcal{F}^{+}$-semantics in Definition 3.9 . The resulting $b$-bounded $\mathcal{F}^{+}$-semantics consists of the $b$-bounded semantics and the $\mathcal{F}^{+}$-semantics extended with all tree failures $(w, X)$ where $w$ is a trace of bound $d_{b}$ and $X$ is any subset of the language. 


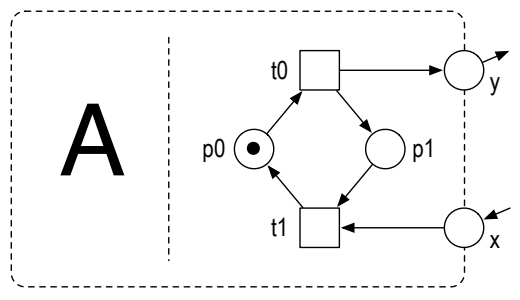

(a) Open net $A^{\prime}$

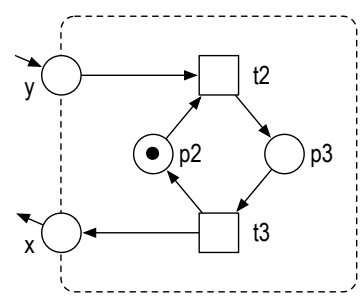

(b) Open net $C$

Fig. 13: Construction for proof of Lemma 6.13

Definition 6.14 (b-bounded $\mathcal{F}^{+}$-semantics). For a labeled net $N$, we define fbound $_{b}(N)=\left\{(w, X) \mid w \in\right.$ bound $\left._{b}(N) \wedge X \in \mathcal{P}\left((I \uplus O)^{+}\right)\right\}$and the b-bounded $\mathcal{F}^{+}{ }_{\text {-semantics of } N \text { by }}$

1. $\operatorname{bound}_{b}(N)$ and

2. $\mathcal{F}_{b}^{+}(N)=\mathcal{F}^{+}(N) \cup$ fbound $_{b}(N)$.

The $\mathcal{F}_{b}^{+}$-refinement relation combines $b$-accordance (see Definition 5.1) and the $\mathcal{F}^{+}$-refinement relation (see Definition 3.14). It is a pleasant surprise that this combination works.

Definition 6.15 $\left(\mathcal{F}_{b}^{+}\right.$-refinement). For two interface-equivalent labeled nets Impl and Spec, Impl $\mathcal{F}_{b}^{+}$-refines Spec, denoted by Impl $\sqsubseteq_{\mathcal{F}_{b}^{+}}$Spec, if

1. bound $_{b}(\operatorname{Impl}) \subseteq$ bound $_{b}($ Spec $)$ and

2. $\forall(w, X) \in \mathcal{F}_{b}^{+}($Impl $): \exists x \in\{\varepsilon\} \cup \downarrow X:\left(w x, x^{-1} X\right) \in \mathcal{F}_{b}^{+}($Spec $)$.

We say $(w, X)$ is dominated by $\left(w x, x^{-1} X\right)$. For two interface-equivalent open nets Impl and Spec, we define Impl $\sqsubseteq_{\mathcal{F}_{b}^{+}} S p e c$, if env $(\operatorname{Impl}) \sqsubseteq_{\mathcal{F}_{b}^{+}}$env $($Spec $)$.

Example 6.5. Consider again the open nets $D$ and $D^{\prime}$. For any bound $b$, we have $(s f, \emptyset) \in \mathcal{F}_{b}^{+}\left(D^{\prime}\right)$ but $(s f, \emptyset) \notin \mathcal{F}_{b}^{+}(D)$; thus, $D^{\prime}$ does not $\mathcal{F}_{b}^{+}$-refine $D$.

The next lemma shows that the $b$-bounded $\mathcal{F}^{+}$-semantics refines the $b$-bounded semantics as it should in view of Lemma 6.13,

Lemma 6.16 $\left(\mathcal{F}_{b}^{+}\right.$-refinement implies language inclusion). For labeled nets Impl and Spec, we have

$$
\text { Impl } \sqsubseteq_{\mathcal{F}_{b}^{+}} \text {Spec implies } L_{b}(\text { Impl }) \subseteq L_{b}(\text { Spec }) .
$$

Proof. Let $w \in L_{b}(\operatorname{Impl})$. Then $(w, \emptyset) \in \mathcal{F}_{b}^{+}(\operatorname{Impl})$ by Definition 6.14, and $(w, \emptyset) \in \mathcal{F}_{b}^{+}($Spec $)$by Definition 6.15 which immediately implies $w \in L_{b}($ Spec $)$ by Definition 6.14 
If we consider the composition of two open nets $N_{1}$ and $N_{2}$, then its $b$ bounded $\mathcal{F}^{+}$-semantics coincides with that of the parallel composition of the two environments, env $\left(N_{1}\right) \Uparrow \operatorname{env}\left(N_{2}\right)$.

Lemma 6.17. For composable open nets $N_{1}$ and $N_{2}$, we have

$$
\mathcal{F}_{b}^{+}\left(N_{1} \oplus N_{2}\right)=\mathcal{F}_{b}^{+}\left(\operatorname{env}\left(N_{1}\right) \Uparrow \operatorname{env}\left(N_{2}\right)\right) .
$$

Proof. Follows directly from Lemma 2.12. If one net has a bound $b_{b}$-violator $w$ due to marking $m$, then the other net can reach an agreeing marking $m^{\prime}$ with trace $w$; thus $w$ is also a bound $_{b}$-violator for the other net (where, if the latter is $e n v\left(N_{1}\right) \Uparrow e n v\left(N_{2}\right)$, we fire 'interface transitions' to make the markings agree strongly if necessary). Likewise, if one net has a tree failure $(w, X)$ due to marking $m$, then the other net can reach an agreeing marking $m^{\prime}$ with trace $w$. If $w$ is a bound $_{b}$-violator in the other net, then $X$ is a refusal set by definition of the $b$ bounded $\mathcal{F}^{+}$-semantics. Otherwise, if some trace $v \in X$ could be performed from $m^{\prime}$, this would also be possible from $m$ due to bisimilarity, yielding a contradiction. Thus, $(w, X)$ is also a tree failure of the other net.

We want to show that $\mathcal{F}_{b}^{+}$-refinement is a precongruence on open nets for composition operator $\oplus$, and for this we will use the precongruence results for $\mathcal{F}^{+}$-refinement and $b$-accordance. First, we characterize the $b$-bounded $\mathcal{F}^{+}$semantics for labeled net composition and hiding, and then combine these results for open net composition.

Lemma 6.18 ( $b$-bounded $\mathcal{F}^{+}$-semantics for labeled net composition). For composable labeled nets $N_{1}$ and $N_{2}$, we have

$$
\begin{aligned}
& \mathcal{F}_{b}^{+}\left(N_{1} \| N_{2}\right)=\left\{(w, X) \mid \exists\left(w_{1}, X_{1}\right) \in \mathcal{F}_{b}^{+}\left(N_{1}\right),\left(w_{2}, X_{2}\right) \in \mathcal{F}_{b}^{+}\left(N_{2}\right):\right. \\
& w \in w_{1} \| w_{2} \wedge \forall x \in X: \\
& \left.x \in x_{1} \| x_{2} \text { implies } x_{1} \in X_{1} \vee x_{2} \in X_{2}\right\} \\
& \cup \operatorname{fbound}_{b}\left(N_{1} \| N_{2}\right) \text {. }
\end{aligned}
$$

Proof. We write $E$ for $N_{1} \| N_{2}$.

$\subseteq$ : Let $(w, X) \in \mathcal{F}_{b}^{+}(E)$. If $w$ is not a bound $b_{b}$ violator of $E$, then $(w, X) \in$ $\mathcal{F}^{+}(E)$ by Definition 6.14 and we conclude with Proposition 3.11 and Definition 6.14 that it is contained in the first set on the right hand side. If $w$ is a bound $d_{b}$-violator of $E$, then $(w, X) \in$ fbound $_{b}(E)$ by Definition 6.14 .

$\supseteq$ : Let $i=1,2$. If both $\left(w_{i}, X_{i}\right) \in \mathcal{F}^{+}\left(N_{i}\right)$, then $(w, X) \in \mathcal{F}^{+}(E)$ by Proposition 3.11 and $\mathcal{F}^{+}(E) \subseteq \mathcal{F}_{b}^{+}(E)$. Assume now that at least one tree failure $\left(w_{i}, X_{i}\right)$ is not contained in the respective $\mathcal{F}^{+}$-semantics. Then trace $w_{i}$ is a bound $d_{b}$-violator by Definition 6.14 and so is $w$ by Proposition 5.3(1), because $w_{3-i} \in L_{b}\left(N_{3-i}\right)$ as argued in the proof of Lemma 6.16. Thus, $(w, X) \in$ fbound $_{b}(E) \subseteq \mathcal{F}_{b}^{+}(E)$ due to Definition 6.14. Furthermore, fbound $(E) \subseteq \mathcal{F}_{b}^{+}(E)$ by Definition 6.14

In the next lemma, we consider a labeled net $N / A, A \subseteq \Sigma$ and we use $\phi(w)$ to denote $\left.w\right|_{\Sigma \backslash A}$. We canonically extend the notion of $\phi(w)$ pointwise to sets of traces. 
Lemma 6.19. For any labeled net $N$ and any label set $A \subseteq \Sigma_{N}^{*}$, we have

$$
\mathcal{F}_{b}^{+}(N / A)=\left\{(\phi(w), X) \mid\left(w, \phi^{-1}(X)\right) \in \mathcal{F}_{b}^{+}(N)\right\} .
$$

Proof. Follows by Proposition 5.3(5) and Proposition 3.12.

Recall Proposition 5.3.(7), which shows how to determine bound $_{b}\left(N_{1} \oplus N_{2}\right)$ from the bound $_{b^{-}}$and the $L_{b}$-semantics of the components; in our setting, we can read off the $L_{b}$-semantics from the $b$-bounded $\mathcal{F}^{+}$-semantics; see the proof of Lemma 6.16. The next proposition similarly characterizes the $b$-bounded $\mathcal{F}^{+}$semantics for open net composition, also using the $b$-bounded semantics. In the proofs of the following two results, we will denote the first set on the right-hand side in Lemma 6.18 by $\mathcal{F} 1_{b}^{+}\left(N_{1}, N_{2}\right)$.

Proposition 6.20 (b-bounded $\mathcal{F}^{+}$-semantics for open net composition). For composable open nets $N_{1}$ and $N_{2}$, we have

$$
\begin{aligned}
& \mathcal{F}_{b}^{+}\left(N_{1} \oplus N_{2}\right)=\left\{(w, X) \mid \exists\left(w_{1}, X_{1}\right) \in \mathcal{F}_{b}^{+}\left(N_{1}\right),\left(w_{2}, X_{2}\right) \in \mathcal{F}_{b}^{+}\left(N_{2}\right):\right. \\
& w \in w_{1} \Uparrow w_{2} \wedge \forall x \in X: \\
& \left.x \in x_{1} \Uparrow x_{2} \text { implies } x_{1} \in X_{1} \vee x_{2} \in X_{2}\right\} \\
& \cup \text { fbound }_{b}\left(N_{1} \oplus N_{2}\right) \text {. }
\end{aligned}
$$

Proof. According to Lemma 6.17, we can consider $\mathcal{F}_{b}^{+}\left(e n v\left(N_{1}\right) \Uparrow e n v\left(N_{2}\right)\right)$ instead of $\mathcal{F}_{b}^{+}\left(N_{1} \oplus N_{2}\right)$. Because $\Uparrow$ is $\|$ followed by hiding, we can determine this set by applying hiding (according to Proposition 5.3.(5) and Lemma 6.19) to the right-hand side of Lemma 6.18 As a result, $\mathcal{F} 1_{b}^{+}\left(N_{1}, N_{2}\right)$ turns into the first set in the present proposition, just as Proposition 3.13 results from Proposition 3.11 in combination with Proposition 3.12. More easily, fbound $\left(N_{1} \| N_{2}\right)$ is analogously translated into fbound $d_{b}\left(N_{1} \oplus N_{2}\right)$ according to Proposition 5.3(1) and $5.3(7)$.

Next, we show that $\mathcal{F}_{b}^{+}$-refinement is a precongruence on labeled nets for the composition operator $\|$. We will use the following three saturation conditions, which also hold for the $\mathcal{F}^{+}$-semantics [29]:

SAT1: $(w, X) \in \mathcal{F}_{b}^{+}(N), X^{\prime} \subseteq X$ implies $\left(w, X^{\prime}\right) \in \mathcal{F}_{b}^{+}(N)$

SAT2: $(w, X) \in \mathcal{F}_{b}^{+}(N) \wedge \forall z \in Z:\left(w z, z^{-1} X\right) \notin \mathcal{F}_{b}^{+}(N)$ implies $(w, X \cup Z) \in \mathcal{F}_{b}^{+}(N)$

SAT3: $(w, X) \in \mathcal{F}_{b}^{+}(N)$ implies $(w, \uparrow X) \in \mathcal{F}_{b}^{+}(N)$

In [29, $\|$ is defined directly on sets of tree failures, taking essentially the equation in Proposition 3.11 as definition. Then, just from the saturation conditions, it is shown that $\mathcal{F}^{+}$-refinement (defined as in Definition 6.15(2)) is a precongruence for $\|$. We will make use of this, although this defining equation does not match Lemma 6.18, but just gives $\mathcal{F} 1_{b}^{+}\left(N_{1}, N_{2}\right)$.

Lemma 6.21. $\mathcal{F}_{b}^{+}$-refinement is a precongruence on labeled nets for the composition operator $\|$. 
Proof. To see the three saturation conditions, consider first some $(w, X) \in$ $\mathcal{F}^{+}(N) \subseteq \mathcal{F}_{b}^{+}(N)$. Then, SAT1 and SAT3 follow directly from [29], as does SAT2 once we observe that $\left(w z, z^{-1} X\right) \notin \mathcal{F}_{b}^{+}(N)$ implies $\left(w z, z^{-1} X\right) \notin \mathcal{F}^{+}(N)$. Second, consider a tree failure $(w, X)$ with $w \in \operatorname{bound}_{b}(N)$; here, all three conditions are immediate because $(w, Y) \in \mathcal{F}_{b}^{+}(N)$ for any $Y \in \mathcal{P}\left((I \uplus O)^{+}\right)$.

Now let Impl $\sqsubseteq_{\mathcal{F}_{b}^{+}}$Spec and $C$ be a composable labeled net for $I m p l$ and Spec. We have to check the two items of Definition 6.15 in order to prove that $\operatorname{Impl}\left\|C \sqsubseteq \mathcal{F}_{b}^{+} \operatorname{Spec}\right\| C$.

The first item follows from Proposition 5.3(1) because our assumption implies bound $_{b}(\operatorname{Impl}) \subseteq$ bound $_{b}($ Spec $)$ and - due to Lemma 6.16- $L_{b}(\operatorname{Impl}) \subseteq L_{b}($ Spec $)$.

For the second item, we first consider some $(w, X) \in \mathcal{F} 1_{b}^{+}(\operatorname{Impl}, C)$. We observe that, due to Definition 6.15 (2), $\mathcal{F}_{b}^{+}(\operatorname{Impl})$ is related to $\mathcal{F}_{b}^{+}($Spec $)$in the sense of $\mathcal{F}^{+}$-refinement. Thus, according to the precongruence result of [29], $\exists x \in\{\varepsilon\} \cup \downarrow X:\left(w x, x^{-1} X\right) \in \mathcal{F} 1_{b}^{+}($Spec,$C) \subseteq \mathcal{F}_{b}^{+}($Spec $\| C)$. Second, we consider some $(w, X) \in$ fbound $_{b}(\operatorname{Impl} \| C)$. This time, due to bound $(\operatorname{Impl} \| C) \subseteq$ bound $_{b}\left(\right.$ Spec $\left._{\| C}\right)$, we even have $(w, X) \in$ fbound $_{b}(\operatorname{Spec} \| C) \subseteq \mathcal{F}_{b}^{+}($Spec $\| C)$ - that is, Definition 6.15(2) is satisfied taking $x=\varepsilon$.

We show that $\mathcal{F}_{b}^{+}$-refinement for labeled nets is preserved under hiding.

Lemma 6.22. $\mathcal{F}_{b}^{+}$-refinement is a precongruence on labeled nets for hiding.

Proof. Let Impl and Spec be labeled nets with Impl $\sqsubseteq_{\mathcal{F}^{+}}$Spec, and $A \subseteq$ $\Sigma^{*}$. Then Proposition 5.3(5) directly implies Definition 6.15(1) for Impl/A and Spec/A. Furthermore, the characterization in Lemma 6.19 corresponds to the defining equation for hiding in [29], so Definition 6.15(2) is inherited from the precongruence result in 29] for $\mathcal{F}^{+}$-refinement and hiding.

Now we directly get the first main result of this section that also the $\mathcal{F}_{b}^{+}$refinement relation is a precongruence for composition operator $\oplus$.

Theorem 6.23 (precongruence). $\mathcal{F}_{b}^{+}$-refinement is a precongruence for the composition operator $\oplus$.

Proof. This is now completely analogous to the proof of Theorem 3.15, except that here the semantics associates two sets with a net and we use Lemma 6.21 and 6.22 on the basis of Lemma 6.17 and Lemma 5.4

With the next theorem, we prove that the coarsest precongruence which is contained in the $b r$-accordance and $\mathcal{F}_{b}^{+}$-refinement coincide.

Theorem 6.24 (precongruence and $\mathcal{F}_{b}^{+}$-refinement coincide). For two interface-equivalent open nets Impl and Spec, we have

$$
\operatorname{Impl} \sqsubseteq_{b r, a c c}^{c} \text { Spec iff Impl } \sqsubseteq_{\mathcal{F}_{b}^{+}} \text {Spec . }
$$


Proof. $\Rightarrow$ : By Lemma $6.13 . \sqsubseteq_{b r, a c c}^{c}$ implies $\sqsubseteq_{b, a c c}$ from which we conclude bound $d_{b^{-}}$ inclusion. It remains to show $\mathcal{F}_{b}^{+}$-inclusion. Let $(w, X) \in \mathcal{F}_{b}^{+}(\operatorname{Impl})$. If $w \in$ bound $_{b}(\operatorname{Impl}) \subseteq$ bound $_{b}\left(\right.$ Spec) (by bound -inclusion), then $(w, X) \in \mathcal{F}_{b}^{+}($Spec $)$ and we are done. Otherwise, we use net $N$ in Fig. 6 and the open net $C$ as in the proof of (the reverse implication of) Theorem 3.17. Following the argumentation in the proof of Theorem 3.17, we have that $C$ is not a $b r$-controller of $I m p l \oplus N$ so it is not a $b r$-controller of $\operatorname{Spec} \oplus N$. We distinguish three cases: If $w \in$ bound $_{b}($ Spec $)$, then $(w, X) \in \mathcal{F}_{b}^{+}($Spec $)$by Definition 6.14. If $w u \in$ bound $_{b}($ Spec $)$ with $u \in \downarrow X$, then $\left(w u, u^{-1} X\right) \in \mathcal{F}_{b}^{+}$(Spec) by Definition 6.14. Otherwise, we use the argumentation in the proof of Theorem 3.17 to conclude that for some $u,\left(w u, u^{-1} X\right) \in \mathcal{F}^{+}($Spec $) \subseteq \mathcal{F}_{b}^{+}($Spec $)$.

$\Leftarrow$ : We show that $I m p l \sqsubseteq \mathcal{F}_{b}^{+}$Spec implies bound $d_{b}$-inclusion, $L_{b}$-inclusion and stop $_{b}$-inclusion from which we conclude by Theorem 6.7 that Impl $\sqsubseteq_{b r, a c c}$ Spec. The latter, in turn, also shows that $\operatorname{Impl} \sqsubseteq_{\mathcal{F}_{b}^{+}}$Spec implies Impl $\sqsubseteq_{b r, a c c}^{c}$ Spec with Theorem 6.23 and the definition of $\sqsubseteq_{b r, a c c}^{c}$.

bound $_{b}$-inclusion follows directly from Definition 6.15, and $L_{b}$-inclusion follows from Lemma 6.16. It remains to show $s \mathrm{~s}_{b}$-inclusion: Let $O$ be the set of output places of Impl and, equivalently, of Spec. We have $w \in \operatorname{stop}_{b}(\mathrm{Impl})$ iff $(w, O) \in \mathcal{F}_{b}^{+}(\operatorname{Impl})$ by Definition 6.14 Then $\operatorname{Impl} \sqsubseteq_{\mathcal{F}_{b}^{+}} \operatorname{Spec}$ implies $(w, O) \in$ $\mathcal{F}_{b}^{+}$(Spec), thus $w \in \operatorname{stop}_{b}($ Spec $)$.

Note that stop-inclusion does not hold because $(w, O) \in \mathcal{F}_{b}^{+}($Spec $)$does not imply $w \in \operatorname{stop}($ Spec $)$.

Example 6.6. For our example open nets $D$ and $D^{\prime}$, we have shown in Example 6.1 that $D^{\prime} b r$-accords with $D$, but $D^{\prime}$ does not $\mathcal{F}_{b}^{+}$-refine $D$ as illustrated in Example 6.5. As a consequence, $D^{\prime} \sqsubseteq_{b r, a c c}^{c} D$ does not hold.

\subsection{Decidability of $\mathcal{F}_{b}^{+}$-refinement}

In this section, we show that checking $\mathcal{F}_{b}^{+}$-refinement is decidable. For this, we work with the reachability graph $R G(N)$ of a labeled net $N$ and the set $B(N) \subseteq M_{N}$ of bound violations of $N$. Actually, our approach works for arbitrary labeled transition systems and the difference between inputs and outputs does not matter. To stress the generality of our approach and to abstract from the not so relevant details of open nets, we assume we are given an arbitrary LTS $S$ (as used in [29, Theorem 61]) where the state set of $S$ is partitioned into $Q \uplus B(S)$ with a finite set $Q$ of states that are reachable from the initial state $m_{S}$ (i.e., $M_{N}-B(S)$ in $N$ ) and a possibly infinite set $B(S)$ of bad states (i.e., $B(N)$ in $N)$. For such an LTS $S$, we can define bound $b_{b}(S), \mathcal{F}^{+}(S)$ and $\mathcal{F}^{+}$-refinement (as for labeled nets). Let $B \mathcal{F}_{b}^{+}(S)=\left\{(w, X) \mid w \in \operatorname{bound}_{b}(S), X \subseteq(I \cup O)^{+}\right\}$ and $\mathcal{F}_{b}^{+}(S)=\mathcal{F}^{+}(S) \cup B \mathcal{F}_{b}^{+}(S)$; define $\mathcal{F}_{b}^{+}$-refinement as in Definition 6.15.

Checking $\mathcal{F}_{b}^{+}$-refinement entails checking both items of Definition 6.15. The first item of Definition 6.15 that is, checking bound $b_{b}$-inclusion - is decidable because we can represent the languages $\operatorname{bound}_{b}(S)$ as a finite automaton. To 


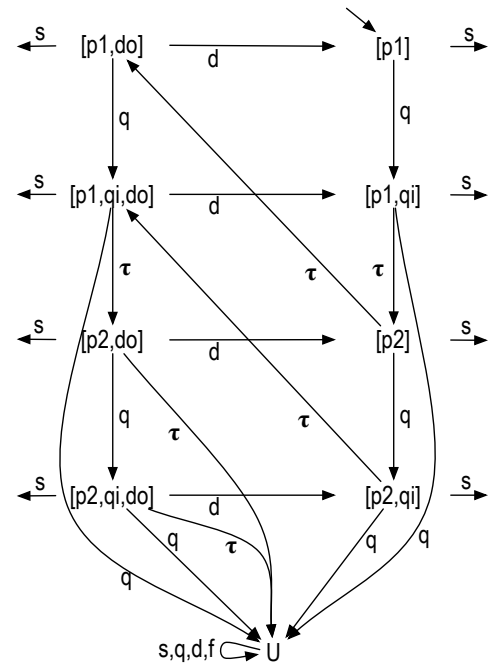

(a) Automaton for bound $d_{1}(D)$

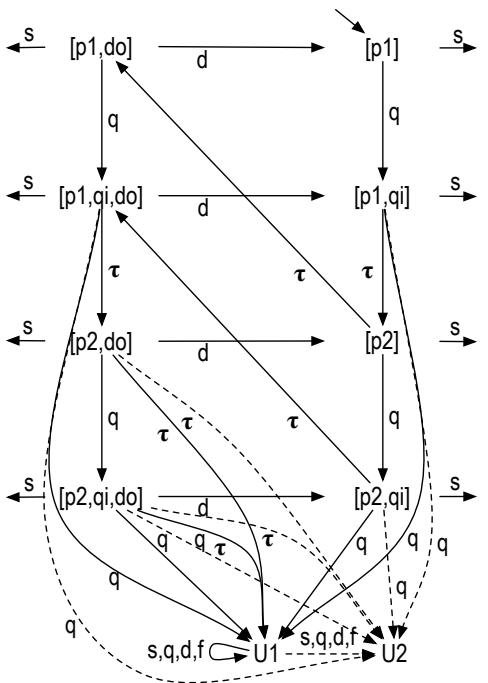

(b) Automaton $U_{12}(D)$

Fig. 14: Sketches of the two finite automata used in the proof of Theorem 6.28. A transition involving $s$ is indicated by an arrow without sink.

this end, we merge all bad states into a single state $U$; more formally, we add a new state $U$ with a self-loop for each action $a \in I \uplus O$, and replace each arc $m \stackrel{a}{\rightarrow} m^{\prime}$ by $m \stackrel{a}{\rightarrow} U$ whenever $m \notin B(S)$ while $m^{\prime} \in B(S)$; finally, we restrict the LTS to the states reachable from $m_{S}$ (which are all in $Q$ ) and consider only $U$ as final state. Now checking bound $_{b}$-inclusion reduces to checking language inclusion for two finite automata.

Example 6.7. Figure 14a sketches the automaton that represents bound $d_{1}(D)$. Parts of the automaton in Fig. 14a that can only be reached with an $s$-transition are not shown. For example, we have $\{q q, q q q q, q q f\} \subseteq$ bound $_{1}(D)$. The marking $\left[p_{1}, q^{i}, q^{i}\right]$ is not 1-bounded; therefore, the transition $\left[p_{1}, q^{i}\right] \stackrel{q}{\rightarrow}\left[p_{1}, q^{i}, q^{i}\right]$ in $R G(\operatorname{env}(D))$ was replaced with $\left[p_{1}, q^{i}\right] \stackrel{q}{\rightarrow} U$. The traces $q q$ and $q q q q$ are strict bound $d_{1}$-violators, as they lead from the initial state $\left[p_{1}\right]$ to the final state $U$ while visiting state $U$ only once (for trace $q q q q$ with three $\tau$-transitions in-between). The traces $q q q q$ and $q q f$ visit $U$ more than once.

To decide refinement of the tree failures - that is, the second item of Definition 6.15 - we shall use the construction of Rensink and Vogler [29, Theorem 61] for deciding $\mathcal{F}^{+}$-refinement for finite-state systems. As checking $\mathcal{F}^{+}$-refinement is known to be decidable [29, Theorem 61], we can conclude that checking $\mathcal{F}_{b}^{+}$refinement is decidable, too.

The automaton we constructed above for $S$ also represents the language $L_{b}(S)$ if we regard all states as final. But it is not suitable for representing 
$\mathcal{F}_{b}^{+}(S)$, since we cannot distinguish the bound $d_{b}$-violators that can be performed from those that have only been added as continuations; thus, the refusal sets are not represented properly. Therefore, we propose a different finite-state representation of $\mathcal{F}_{b}^{+}(S)$ on which checking $\mathcal{F}^{+}$-refinement coincides with checking $\mathcal{F}_{b}^{+}$-refinement for $S$.

We construct the finite LTS $U_{12}(S)$ as follows. The state set of $U_{12}(S)$ is $Q \uplus\left\{U_{1}, U_{2}\right\}$, the start state is $m_{S}, B\left(U_{12}(S)\right)=\left\{U_{1}, U_{2}\right\}$ is the set of bound violations, and the transition relation is defined as

$$
\begin{aligned}
& \left\{m \stackrel{a}{\rightarrow} m^{\prime} \mid m, m^{\prime} \in Q, a \in I \cup O \cup\{\tau\}, m \stackrel{a}{\rightarrow} m^{\prime} \text { in } S\right\} \\
\cup & \left\{m \stackrel{a}{\rightarrow} U_{1}, m \stackrel{\rightarrow}{\rightarrow} U_{2} \mid m \in Q, \exists m^{\prime} \in B(S), a \in I \cup O \cup\{\tau\}: m \stackrel{a}{\rightarrow} m^{\prime} \text { in } S\right\} \\
\cup & \left\{U_{1} \stackrel{a}{\rightarrow} U_{1}, U_{1} \stackrel{a}{\rightarrow} U_{2} \mid a \in I \cup O\right\} .
\end{aligned}
$$

Example 6.8. Figure $14 \mathrm{~b}$ shows a part of the automaton $U_{12}(D)$. For example, we have $(q q q q,\{f\}) \in \mathcal{F}_{b}^{+}(D) \cap \mathcal{F}^{+}\left(U_{12}(D)\right)$, because the trace $q q q q$ may lead to the state $U_{2}$ and then refuse $f$. Further, we have $(q,\{q q f\}) \in \mathcal{F}_{b}^{+}(D) \backslash \mathcal{F}^{+}\left(U_{12}(D)\right)$ observe $f$ can never fire in env $(D)$, while any state reached by $q$ in $U_{12}(D)$ can reach $U_{1}$ with $q q$ and then add $f$. There is $q \in \downarrow\{q q f\} \backslash\{q q f\}$ and the trace $q q$ is a bound $d_{1}$-violator of $D$.

The next lemma, gives three observations about $U_{12}(S)$.

Lemma 6.25. Let $S$ be an LTS. Then the following facts hold for $U_{12}(S)$.

1. $w \in$ bound $_{b}(S)$ iff $m_{N} \stackrel{w}{\Longrightarrow} U_{1}$ iff $m_{N} \stackrel{w}{\Longrightarrow} U_{2}$.

2. $B \mathcal{F}_{b}^{+}(S) \subseteq \mathcal{F}^{+}\left(U_{12}(S)\right)$ (i.e., $U_{2}$ can refuse all $X \subseteq(I \cup O)^{+}$)

3. $\mathcal{F}^{+}\left(U_{12}(S)\right) \subseteq \mathcal{F}_{b}^{+}(S)$ and $(w, X) \in \mathcal{F}_{b}^{+}(S) \backslash \mathcal{F}^{+}\left(U_{12}(S)\right)$ implies $\exists u \in$ $\downarrow X \backslash X$ such that $w u \in$ bound $_{b}(S)$

Proof. The first item follows immediately from the definition of $U_{12}(S)$, and the second item is an implication of the first item. Consider the third item. The sets agree on those $(w, X)$ with $w \in$ bound $_{b}(S)$ by Item 2 . So consider $w \notin$ bound $_{b}(S)$. If $(w, X) \in \mathcal{F}^{+}\left(U_{12}(S)\right)$ due to $m_{S} \stackrel{w}{\Longrightarrow} m$, then we also have $m_{S} \stackrel{w}{\Longrightarrow} m$ in $S$ with the same run. In $U_{12}(S), m$ could only have more traces due to runs using $U_{1}$, so it can only refuse less. Thus, $(w, X) \in \mathcal{F}^{+}(S)$ and inclusion follows.

If $(w, X) \in \mathcal{F}_{b}^{+}(S)$ due to $m$, but $(w, X) \notin \mathcal{F}^{+}\left(U_{12}(S)\right)$, then this must be due to a run from $m$ that touches $U_{1}$. Assume this happens for the first time after $u \neq \varepsilon$; that is, we have $m_{N} \stackrel{w}{\Longrightarrow} m \stackrel{u}{\Longrightarrow} U_{1} \stackrel{u^{\prime}}{\Longrightarrow}$ with $u u^{\prime} \in X$. Thus, $w u \in$ bound $_{b}(S)$ and $u \in \downarrow X$. As $m_{N} \stackrel{w}{\Longrightarrow} m \stackrel{u}{\Longrightarrow}$ also in $S$, we further have $u \notin X$.

The following lemma gives another prerequisite for the decidability proof.

Lemma 6.26. For $X, Y \in \mathcal{P}\left(\Sigma^{*}\right)$, let $x \in \downarrow Y \cup\{\varepsilon\}$ and $X=x^{-1} Y$. Then

1. $u \in \downarrow X$ implies $x u \in \downarrow Y$

2. $u \notin X$ implies $x u \notin Y$ 
Proof. (1) Due to $u u^{\prime} \in X$ and $x u u^{\prime} \in Y$.

(2) Suppose $x u \in Y$. Then $u \in X$ and we have a contradiction.

With the next lemma and the subsequent theorem, we return to speaking about open nets - though we could as well talk about two LTS. The lemma shows that deciding $\mathcal{F}_{b}^{+}$-refinement for two open nets $\mathrm{Impl}$ and $\mathrm{Spec}$ reduces to checking $\mathcal{F}^{+}$-refinement of their automata $U_{12}($ Spec $)$ and $U_{12}(\operatorname{Impl})$.

Lemma 6.27. For two open nets Impl and Spec such that bound $(\operatorname{Impl}) \subseteq$ bound $_{b}($ Spec $)$, we have

$$
\text { Impl } \sqsubseteq_{\mathcal{F}_{b}^{+}} \text {Spec iff } U_{12}(\operatorname{Impl}) \sqsubseteq_{\mathcal{F}^{+}} U_{12}(\text { Spec }) .
$$

Proof. $\Rightarrow$ : Each $(v, Y) \in \mathcal{F}^{+}\left(U_{12}(\operatorname{Impl})\right) \subseteq \mathcal{F}_{b}^{+}(\operatorname{Impl})$ (by Lemma 6.25(3)) is dominated by some $(w, X) \in \mathcal{F}_{b}^{+}$(Spec) due to some $x \in \downarrow Y \cup\{\varepsilon\}$, i.e., $X=$ $x^{-1} Y, w=v x$. If $(w, X) \in \mathcal{F}^{+}\left(U_{12}(\right.$ Impl $\left.)\right)$, we are done. So assume otherwise and consider $u \in \downarrow X \backslash X$ according to Lemma 6.25(3). Then $x u \in \downarrow Y \backslash Y$ by Lemma 6.26 and $\left(v x u,(x u)^{-1} Y\right) \in \mathcal{F}^{+}\left(U_{12}(\right.$ Spec $\left.)\right)$ because $v x u \in$ bound $_{b}($ Spec $)$ and $\varepsilon \notin(x u)^{-1} Y$. Hence, $(v, Y)$ is also covered in this case.

$\Leftarrow: \operatorname{Each}(w, X) \in \mathcal{F}^{+}\left(U_{12}(\right.$ Impl $\left.)\right)$ is dominated by $\mathcal{F}^{+}\left(U_{12}(\right.$ Spec $\left.)\right) \subseteq \mathcal{F}_{b}^{+}($Spec $)$ by Lemma 6.25 (3). So consider $(w, X) \in \mathcal{F}_{h}^{+}(\operatorname{Impl}) \backslash \mathcal{F}^{+}\left(U_{12}(\right.$ Impl $\left.)\right)$ and respectively $u \in \downarrow X \backslash X$ according to Lemma 6.25(3). Then, $\left(w u, u^{-1} X\right) \in \mathcal{F}_{b}^{+}($Spec $)$ because $w u \in$ bound $_{b}(\operatorname{Impl}) \subseteq$ bound $_{b}($ Spec $)$ and $\varepsilon \notin u^{-1} X$. Thus, $(w, X)$ is also dominated in this case.

With Lemma 6.27, we have shown:

Theorem $6.28\left(\mathcal{F}_{b}^{+}\right.$-refinement is decidable). For two interface-equivalent open nets Impl and Spec, checking whether Impl $\sqsubseteq_{\mathcal{F}_{b}^{+}}$Spec is decidable.

\section{Bounded nets and final markings}

In this section, we consider open nets with final markings and whose composition is bounded. We refer to this notion of responsiveness as $b f$-responsiveness.

Definition 7.1 ( $b f$-responsiveness). Let $N_{1}$ and $N_{2}$ be composable open nets. A marking of $N_{1} \oplus N_{2}$ is $b f$-responsive if it is $f$-responsive and $b$-bounded. Open nets $N_{1}$ and $N_{2}$ are $b f$-responsive if their composition $N_{1} \oplus N_{2}$ is a closed net and every reachable marking in $N_{1} \oplus N_{2}$ is bf-responsive.

Two open nets are $b f$-responsive if and only if they are $f$-responsive and their composition is $b$-bounded. In the presence of final markings, we can prove a stronger semantical characterization of responsiveness than in Proposition 6.2. Due to $b f$-responsiveness each net has always the chance to send a message or the composition terminates. 


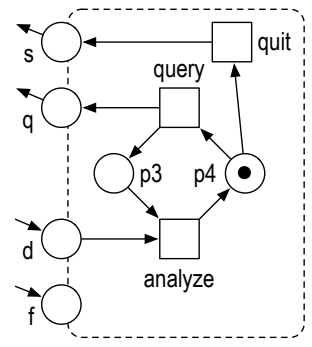

(a) Open net $U^{\prime}$

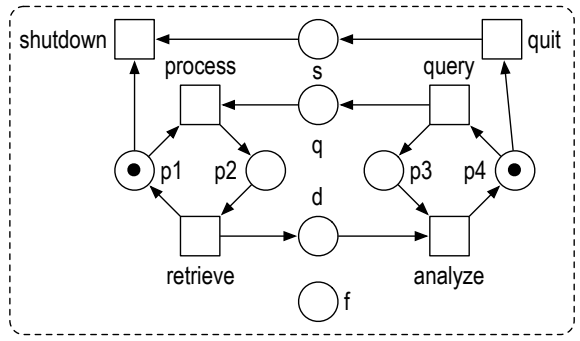

(b) Open net $D \oplus U^{\prime}$

Fig. 15: Open net $U^{\prime}$ modeling another user of open net $D$ in Fig. 8 a and their composition $D \oplus U^{\prime}$. In addition to the models, we have $\Omega_{U^{\prime}}=\Omega_{D \oplus U^{\prime}}=\{[]\}$.

Proposition 7.2. Let open nets $N_{1}$ and $N_{2}$ be bf-responsive. Then, from any reachable marking $m$, markings $m_{1}$ and $m_{2}$ are reachable such that $m_{1} \stackrel{t_{1}}{\longrightarrow}$ with $t_{1}^{\bullet} \cap O_{1} \neq \emptyset$ and $m_{2} \stackrel{t_{2}}{\longrightarrow}$ with $t_{2}^{\bullet} \cap O_{2} \neq \emptyset$, or a final marking is reachable.

Proof. Direct consequence of Proposition 6.2 and Definitions 7.1 and 4.1 .

Again, we redefine the notion of a controller and of accordance for this variant of responsiveness to $b f r$-controller and $b f r$-accordance. Also $b f r$-accordance shall turn out not to be a precongruence; thus, we also introduce its coarsest precongruence.

Definition 7.3 ( $b f r$-controller, $b f r$-accordance). An open net $C$ is a $b f r$ controller of an open net $N$ if $N$ and $C$ are $b f$-responsive.

For interface-equivalent open nets Impl and Spec, Impl bfr-accords with Spec, denoted by $I m p l \sqsubseteq_{b f r, a c c} S p e c$, if for all open nets $C$ holds: $C$ is a $b f r$-controller of Spec implies $C$ is a bfr-controller of Impl.

We denote the coarsest precongruence contained in $\sqsubseteq_{b f r}, a c c$ by $\sqsubseteq_{b f r, a c c}^{c}$.

Example 7.1. The open net $U^{\prime}$ in Fig. 15a represents another user of the database server $D$ in Fig. 8a. It has the same functionality as the open net $U$ in Fig. 8b, but may additionally decide to quit and shut down the database (output place $s$ ). The open nets $D$ and $U^{\prime}$ are composable; their composition $D \oplus U^{\prime}$ is a closed net, which is depicted in Fig. 15b. $U^{\prime}$ is not a $b r$-controller of $D$ because the nonresponsive marking [] is reachable in $D \oplus U^{\prime}$, but $U^{\prime}$ is a $b f r$-controller of $D$ because [] is a final marking of $D \oplus U^{\prime}$. Moreover, $U^{\prime}$ is not a $b r$-controller of $D^{\prime}$ because $D^{\prime} \oplus U^{\prime}$ can reach the non- $b f$-responsive marking $[f]$ (see Proposition 7.2). Thus, although $D^{\prime} b r$-accords with $D$ (see Example 6.1) it does not $b f r$-accord with $D$.

As shown in Example 6.1, $D$ br-accords with $D^{\prime}$. No bfr-controller of $D^{\prime}$ can send $s$ as otherwise this can lead to the marking $[f]$. Thus, we also have $D$ bfr-accords with $D^{\prime}$. 
Like the previous responsiveness variants, bounded final accordance is not a precongruence either. $D$ bfr-accords with $D^{\prime}$, but $D \oplus Y$ does not $b f r$-accord with $D^{\prime} \oplus Y$, because open net $X$ is a bfr-controller of $D^{\prime} \oplus Y$ but not of $D \oplus Y$ (see Fig. 10 .

We continue by giving a trace-based semantics for $b f$-responsiveness. Then, we formalize the coarsest precongruence, which is contained in the $b f r$-accordance relation.

\subsection{A trace-based semantics for bounded final responsiveness}

Our trace-based semantics for $b f$-responsiveness of an open net $N$ combines the stopdead-semantics of Definition 4.1 and the $b$-bounded stop-semantics of Definition 6.1. The resulting b-bounded stopdead-semantics consists of four sets of traces: bound $_{b}$-violators, the language, stop-traces, and dead-traces.

Definition 7.4 (b-bounded stopdead-semantics). The b-bounded stopdeadsemantics of a labeled net $N$ is defined by the sets of traces

- $\operatorname{bound}_{b}(N)$,

$-L_{b}(N)$,

$-\operatorname{stop}_{b}(N)$, and

$-\operatorname{dead}_{b}(N)=\operatorname{dead}(N) \cup \operatorname{bound}_{b}(N)$.

For the purpose of characterizing $b f r$-controllers, we could also work with a variant of the $b$-bounded stopdead-semantics that contains stop-traces and dead-traces instead of the flooded version. This is similar to the characterization of $b r$-controllers in Sect. 6.1. Still, we use $s_{\text {top }}$-traces and dead $d_{b}$-traces in this semantics because it gives a better sufficient condition for $b f r$-accordance in Theorem 7.7 below.

Lemma 7.5. For composable open nets $N_{1}$ and $N_{2}$ with $L_{b}\left(N_{1}\right) \cap$ bound $d_{b}\left(N_{2}\right)=$ $\emptyset$ and bound $\left(N_{1}\right) \cap L_{b}\left(N_{2}\right)=\emptyset$, we have

$$
\begin{array}{lll}
\operatorname{stop}\left(N_{1}\right) \cap \operatorname{dead}\left(N_{2}\right)=\emptyset \text { and } & \text { iff } & \operatorname{stop}_{b}\left(N_{1}\right) \cap \operatorname{dead}_{b}\left(N_{2}\right)=\emptyset \text { and } \\
\operatorname{dead}\left(N_{1}\right) \cap \operatorname{stop}\left(N_{2}\right)=\emptyset & & \operatorname{dead}_{b}\left(N_{1}\right) \cap \operatorname{stop}_{b}\left(N_{2}\right)=\emptyset .
\end{array}
$$

Proof. $\Leftarrow$ : Follows immediately from Definition 7.4

$\Rightarrow$ : We can write $\operatorname{stop}_{b}\left(N_{1}\right) \cap \operatorname{dead}_{b}\left(N_{2}\right)$ as a union of four intersections

$$
\begin{aligned}
\operatorname{stop}_{b}\left(N_{1}\right) \cap \operatorname{dead}_{b}\left(N_{2}\right)= & \left(\operatorname{stop}\left(N_{1}\right) \cap \operatorname{dead}\left(N_{2}\right)\right) \\
& \cup\left(\operatorname{bound}\left(N_{1}\right) \cap \operatorname{dead}\left(N_{2}\right)\right) \\
& \cup\left(\operatorname{stop}\left(N_{1}\right) \cap \operatorname{bound}_{b}\left(N_{2}\right)\right) \\
& \cup\left(\operatorname{bound}_{b}\left(N_{1}\right) \cap \operatorname{bound}_{b}\left(N_{2}\right)\right),
\end{aligned}
$$

all of which are empty:

- $\operatorname{stop}\left(N_{1}\right) \cap \operatorname{dead}\left(N_{2}\right)=\emptyset$ by assumption 
- bound $_{b}\left(N_{1}\right) \cap \operatorname{dead}\left(N_{2}\right)=\emptyset$, by bound $\left(N_{1}\right) \cap L_{b}\left(N_{2}\right)=\emptyset$ (Corollary 5.5) and $\operatorname{dead}\left(N_{2}\right) \subseteq L\left(N_{2}\right) \subseteq L_{b}\left(N_{2}\right)$

$-\operatorname{stop}\left(N_{1}\right) \cap$ bound $_{b}\left(N_{2}\right)=\emptyset$, by $L_{b}\left(N_{1}\right) \cap$ bound $_{b}\left(N_{2}\right)=\emptyset$ (Corollary 5.5) and $\operatorname{stop}\left(N_{1}\right) \subseteq L\left(N_{1}\right) \subseteq L_{b}\left(N_{1}\right)$

- bound $\left(N_{1}\right) \cap$ bound $_{b}\left(N_{2}\right)=\emptyset$, by bound $\left(N_{1}\right) \cap L_{b}\left(N_{2}\right)=\emptyset$ (Corollary 5.5) and bound $_{b}\left(N_{2}\right) \subseteq L_{b}\left(N_{2}\right)$

A similar argument applies to $\operatorname{dead}_{b}\left(N_{1}\right) \cap \operatorname{stop}_{b}\left(N_{2}\right)=\emptyset$.

An open net $C$ is a $b f r$-controller of an open net $N$ if and only if it is an $f r$-controller and a $b$-controller of $N$. Thus, by Proposition 4.6 and Corollary 5.5 in combination with Lemma 7.5 , we give the following characterization of $b f$ responsiveness.

Proposition 7.6 ( $b f$-responsiveness vs. $b$-bounded stopdead-semantics). Let $N_{1}$ and $N_{2}$ be composable open nets such that $N_{1} \oplus N_{2}$ is a closed net. Then

$$
N_{1} \text { and } N_{2} \text { are bf-responsive iff } \begin{aligned}
& \operatorname{stop}_{b}\left(N_{1}\right) \cap \operatorname{dead}_{b}\left(N_{2}\right)=\emptyset \text { and } \\
& \operatorname{dead}_{b}\left(N_{1}\right) \cap \operatorname{stop}_{b}\left(N_{2}\right)=\emptyset \text { and } \\
& \\
& \\
& L_{b}\left(N_{1}\right) \cap \text { bound }_{b}\left(N_{2}\right)=\emptyset \text { and } \\
& \text { bound }_{b}\left(N_{1}\right) \cap L_{b}\left(N_{2}\right)=\emptyset .
\end{aligned}
$$

Inclusion of the $b$-bounded stopdead-semantics defines a refinement relation which implies $b f$-responsiveness.

Theorem 7.7 (b-bounded stop dead-inclusion implies $b f r$-accordance). For two interface-equivalent open nets Impl and Spec, we have

$$
\begin{aligned}
& \text { bound }_{b}(\text { Impl }) \subseteq \text { bound }_{b}(\text { Spec }) \text { and } \\
& L_{b}(\text { Impl }) \subseteq L_{b}(\text { Spec }) \text { and } \\
& \text { stop }_{b}(\text { Impl }) \subseteq \operatorname{stop}_{b}(\text { Spec }) \text { and } \\
& \operatorname{dead}_{b}(\text { Impl }) \subseteq \operatorname{dead}_{b}(\text { Spec })
\end{aligned}
$$

Proof. Proof by contraposition. Consider an open net $C$ such that $\operatorname{Impl} \oplus C$ and, equivalently, Spec $\oplus C$ are closed nets. Otherwise, $C$ is neither a $b f r$ controller of $\mathrm{Impl}$ nor of Spec. Assume that $C$ is not a $b f r$-controller of $I m p l$. Then, Impl and $C$ are not $b f$-responsive by Definition 7.3 , and we have either $\operatorname{stop}_{b}\left(\operatorname{Impl} \cap \operatorname{dead}_{b}(C) \neq \emptyset, \operatorname{dead}_{b}(\operatorname{Impl}) \cap \operatorname{stop}_{b}(C) \neq \emptyset, L_{b}(\operatorname{Impl}) \cap \operatorname{bound}_{b}(C) \neq\right.$ $\emptyset$, or bound $(\operatorname{Impl}) \cap L_{b}(C) \neq \emptyset$ by Proposition 7.6. Because of the assumed inclusions, we have either stop $\sin _{b}(\operatorname{Spec}) \cap \operatorname{dead}_{b}(C) \neq \emptyset$, $\operatorname{dead}_{b}(\operatorname{Spec}) \cap \operatorname{stop}_{b}(C) \neq \emptyset$, $L_{b}($ Spec $) \cap$ bound $_{b}(C) \neq \emptyset$, or bound $($ Spec $) \cap L_{b}(C) \neq \emptyset$. Again with Proposition 7.6. we see that $S p e c$ and $C$ are not $b f$-responsive; that is, $C$ is not a bfr-controller of Spec and thus Impl $\sqsubseteq_{b f r, a c c}$ Spec.

As for Theorem 6.7, the converse of Theorem 7.7 does not hold in general either, because bounded final responsiveness considers only the reliable part of open nets. 
Example 7.2. Consider the open nets $D$ and $D^{\prime}$, this time, with the empty sets of final markings. No $b f r$-controller of $D$ will send an $s$ because this would eventually lead to firing of shutdown, yielding a non- $b f$-responsive marking where neither $p_{1}$ nor $p_{2}$ contains a token. Thus, we conclude that $D^{\prime} b f r$-accords with $D$. Now we can apply the same trace $s f$ as in Example 6.2 and obtain $s f \in$ $L_{b}\left(D^{\prime}\right) \backslash L_{b}(D)$.

For this reason, we adapt the coverable $b$-bounded stop-semantics from the previous section.

\subsection{A coverable trace-based semantics for bounded final responsiveness}

We combine the idea of the coverable $b$-bounded stop-semantics in Definition 6.8 and the stopdead-semantics in Definition 4.3 yielding the coverable b-bounded stop dead-semantics. For this, we define the notion of an $b f r$-uncoverable trace. As for the language and the stop-traces in the coverable $b$-bounded stop-semantics, we also flood the dead-traces in our new semantics.

Definition 7.8 (coverable $b$-bounded stopdead-semantics). Let $N$ be an open net. A word $w \in(I \uplus O)^{*}$ is a bfr-uncoverable trace of $N$ if there does not exist a $b f r$-controller $C$ of $N$ with $w \in L_{b}(C)$. The coverable b-bounded stopdead-semantics of $N$ is defined by the sets of traces

- uncov $_{b f r}(N)=\left\{w \in(I \uplus O)^{*} \mid w\right.$ is a bfr-uncoverable trace of $\left.N\right\}$,

- $u L_{b f r}(N)=L(N) \cup$ uncov $_{b f r}(N)$,

- ustop $_{b f r}(N)=\operatorname{stop}(N) \cup$ uncov $_{b f r}(N)$, and

$-\operatorname{udead}_{b f r}(N)=\operatorname{dead}(N) \cup \operatorname{uncov}_{b r}(N)$.

Example 7.3. We showed in Example 7.1 that $D$ bfr-accords with $D^{\prime}$. Now ob-

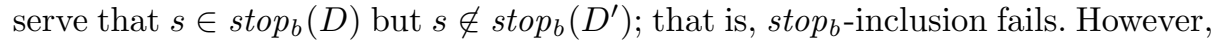
no $b f r$-controller of $D^{\prime}$ has trace $s$ in its language; otherwise, $D^{\prime}$ may reach the non- $b f r$-responsive marking []. Thus, we have $s \in u_{n \operatorname{cov}_{b f r}}\left(D^{\prime}\right)$ and $s$ is also in the flooded stop-set of $D^{\prime}$, i.e., $s \in$ ustop $_{b r}\left(D^{\prime}\right)$.

By Proposition 7.6, a bound $d_{b}$-violator of an open net is an $b f r$-uncoverable trace of $N$. So we directly conclude that the set of bound $d_{b}$-violators of $N$ is contained in the set of $b f r$-uncoverable traces of $N$. As a result, the coverable $b$-bounded stopdead-semantics includes the b-bounded stopdead-semantics.

Lemma 7.9 (b-bounded stopdead-semantics is included). For any open net $N$, we have

- $\operatorname{bound}_{b}(N) \subseteq$ uncov $_{b f r}(N)$,

$-L_{b}(N) \subseteq u L_{b f r}(N)$,

- $\operatorname{stop}_{b}(N) \subseteq$ ustop $_{b f r}(N)$, and

- $\operatorname{dead}_{b}(N) \subseteq \operatorname{udead}_{b f r}(N)$. 
Next, we characterize $b f r$-accordance. For the proof, we restate Lemmata 6.10 and 6.11 for $b f$-responsiveness. In addition, we show that for every trace $w$ of $N$, which is neither a dead-trace nor $b f r$-uncoverable, there exists a $b f r$-controller of $N$ containing $w$ in its set of stop-traces.

Lemma 7.10. Let $N$ be an open net. Then

1. If $w \notin u L_{b f r}(N)$, then there exists a bfr-controller $C$ of $N$ with $w \in$ bound $_{b}(C)$.

2. If $w \in L(N) \backslash$ ustop $_{b f r}(N)$, then there exists a bfr-controller $C$ of $N$ with $w \in \operatorname{dead}(C)$.

3. If $w \in L(N) \backslash$ udead $_{b f r}(N)$, then there exists a bfr-controller $C$ of $N$ with $w \in \operatorname{stop}(C)$.

Proof. (1) This is analogous to the proof of Lemma 6.10 except that $C$ is a bfr-controller of $N$ and we have to consider the final markings. The final markings of $N_{w}^{\text {bound }}$ are $\Omega_{N_{w}^{\text {bound }}}=\left\{[p] \mid p \in\left\{p_{0}, \ldots, p_{k}, p_{\text {err }}\right\}\right\}$. That way we do not add additional dead-traces to $N_{w}^{\text {bound }} \oplus C^{\prime}$ beside continuations of $w \in$ bound $_{b}\left(N_{w}^{\text {bound }} \oplus C^{\prime}\right)$. Clearly, $N_{w}^{\text {bound }} \oplus C^{\prime}$ is still a bfr-controller of $N$ because of $w \notin u L_{b r}(N)$ and Proposition 7.6.

(2) This is analogous to the proof of Lemma 6.11 except that $C$ is a $b f r-$ controller of $N$ and we put $\Omega_{N_{w}}=\left\{[p] \mid p \in\left\{p_{0}, \ldots, p_{k-1}, p_{e r r}\right\}\right\}$. As $\left[p_{k}\right]$ is not a final marking of $N_{w}$, the stop-trace $w$ of $N_{w} \oplus C^{\prime}$ is a dead-trace of $N_{w} \oplus C^{\prime}$.

(3) This is analogous to the proof of Lemma 6.11. except that $C$ is a $b f r-$ controller of $N$ and we have to change $N_{w}$ slightly: The introduced stop-trace $w$ of $N_{w} \oplus C^{\prime}$ may be a stop-trace of $N$, i.e., $w \in \operatorname{stop}(N) \backslash \operatorname{dead}(N)$. Thus, after $N_{w}$ recognizes $w$, we buffer all messages from $C^{\prime}$ to $N_{w}$ inside $N_{w}$ and define all markings of $N_{w}$ and $C^{\prime}$ as final markings. That way, we do not introduce an additional dead-trace of $N_{w} \oplus C^{\prime}$.

Theorem 7.11 (coverable $b$-bounded stopdead-inclusion vs. $b f r$-accordance). For two interface-equivalent open nets Impl and Spec, we have

$$
\begin{aligned}
& \text { uncov }_{b f r}(\operatorname{Impl}) \subseteq \operatorname{uncov}_{b f r}(\text { Spec }), \\
& I m p l \sqsubseteq_{b f r, a c c} \text { Spec } \quad \text { iff } \quad u L_{b f r}(\operatorname{Impl}) \subseteq u L_{b f r}(\text { Spec }), \\
& \text { ustop }_{b f r}(\operatorname{Impl}) \subseteq \text { ustop }_{b f r}(\text { Spec }) \text {, and } \\
& \operatorname{udead}_{b f r}(\operatorname{Impl}) \subseteq \operatorname{udead}_{b f r}(\text { Spec }) .
\end{aligned}
$$

Proof. $\Rightarrow$ : For the first three trace inclusions, we apply the same argumentation as in the proof of Theorem 6.12 by replacing Proposition 6.6, Lemma 6.10, and Lemma 6.11 with Proposition 7.6. Lemma 7.10(1) and(2), respectively.

Let $w \in \operatorname{udead}_{b f r}(\operatorname{Impl}) \backslash$ uncov $_{b f r}(\operatorname{Impl})$ and assume $w \notin \operatorname{udead}_{b f r}(\operatorname{Spec})$. Then, $w \in \operatorname{dead}(\operatorname{Impl}) \subseteq L(\operatorname{Impl})$ and $w \in L($ Spec $)$, as $u L_{b f r}(\operatorname{Impl}) \subseteq u L_{b f r}($ Spec $)$ has been shown already. We can construct a $b f r$-controller $C$ of Spec with $w \in$ stop $(C)$ by Lemma 7.10(3). Clearly, $C$ is not a $b f r$-controller of Impl by Proposition 7.6. and we have a contradiction to our assumption that Impl ${ }_{b f r}, a c c$ Spec. Thus, $w \in$ udead $_{b f r}($ Spec $)$. 
$\Leftarrow$ : Proof by contraposition. Assume that the four inclusions hold and that $C$ is not a $b f r$-controller of $I m p l$. We show that $C$ is not a $b f r$-controller of Spec either.

Impl and $C$ are not $b f$-responsive by Definition 7.3 and we have $\operatorname{stop}_{b}(\operatorname{Impl}) \cap$ $\operatorname{dead}_{b}(C) \neq \emptyset, \operatorname{dead}_{b}(\operatorname{Impl}) \cap \operatorname{stop}_{b}(C) \neq \emptyset, L_{b}(\operatorname{Impl}) \cap \operatorname{bound}_{b}(C) \neq \emptyset$, or bound $_{b}(\operatorname{Impl}) \cap L_{b}(C) \neq \emptyset$ by Proposition 7.6 . We consider each case separately:

$-w \in \operatorname{stop}_{b}(\operatorname{Impl}) \cap \operatorname{dead}_{b}(C)$ : Then, $w \in$ ustop $_{b f r}(\operatorname{Impl}) \subseteq$ ustop $_{b f r}($ Spec $)$ by Lemma 7.9 and assumption. If $w \in \operatorname{stop}($ Spec $)$ then $C$ is not a $b f r$-controller of Spec by Proposition 7.6. otherwise, if $w \in$ uncov $_{b f r}($ Spec $)$, then $C$ is not a bfr-controller of Spec by $w \in \operatorname{dead}_{b}(C) \subseteq L_{b}(C)$ and Definition 7.8.

$-w \in \operatorname{dead}_{b}(\operatorname{Impl}) \cap \operatorname{stop}_{b}(C)$ : Then, $w \in$ udead $_{b f r}(\operatorname{Impl}) \subseteq$ udead dffr $_{\text {(Spec }) \text { by }}$ Lemma 7.9 and assumption. If $w \in \operatorname{dead}($ Spec $)$ then $C$ is not a $b f r$-controller of Spec by Proposition 7.6 otherwise, if $w \in$ uncov $_{b f r}($ Spec $)$, then $C$ is not a bfr-controller of Spec by $w \in \operatorname{stop}_{b}(C) \subseteq L_{b}(C)$ and Definition 7.8 .

- $w \in L_{b}(\operatorname{Impl}) \cap$ bound $_{b}(C)$ or $w \in$ bound $_{b}(\operatorname{Impl}) \cap L_{b}(C)$ : same argumentation as in the proof of Theorem 6.12 by replacing Proposition 6.6 and Lemma 6.9 with Proposition 7.6 and Lemma 7.9 , respectively.

Like the trace set uncov br , also the trace set uncov $b f_{r}$ can be calculated using the notion of a most permissive controller. However, as this calculation is not the focus of this article, we do not show it.

\subsection{Deriving the coarsest precongruence for bounded final responsiveness}

In this section, we shall derive the coarsest precongruence for $b f$-responsiveness. To cope with the combination of $f$-responsiveness and $b$-boundedness, we add information about bound $_{b}$-violators to the $\mathcal{F}_{\text {fin }}^{+}$-semantics (Definition 4.8), similarly as we did to derive the $b$-bounded $\mathcal{F}^{+}$-semantics from the $\mathcal{F}^{+}$-semantics. The resulting semantics is the $b$-bounded $\mathcal{F}_{\text {fin }}^{+}$-semantics, where an element $(w, X, Y)$ is called $b$-bounded fintree failure.

Definition 7.12 (b-bounded $\mathcal{F}_{f i n}^{+}$-semantics). For a labeled net $N$, we define finbound $_{b}(N)=$ bound $_{b}(N) \times \mathcal{P}\left(\Sigma^{+}\right) \times \mathcal{P}\left(\Sigma^{*}\right)$ and the b-bounded $\mathcal{F}_{\text {fin }}^{+}$-semantics of $N$ by

- $\operatorname{bound}_{b}(N)$ and

$-\mathcal{F}_{b, f i n}^{+}(N)=\mathcal{F}_{\text {fin }}^{+}(N) \cup$ finbound $_{b}(N)$.

The $\mathcal{F}_{b, f i n}^{+}$-refinement relation combines the $\mathcal{F}_{\text {fin }}^{+}$-refinement relation (see Definition 4.9) and $b$-accordance (see Definition 5.1).

Definition $7.13\left(\mathcal{F}_{b, f i n}^{+}\right.$-refinement). For two interface-equivalent labeled nets Impl and Spec, Impl $\mathcal{F}_{b, f i n}^{+}$-refines Spec, denoted by Impl $\sqsubseteq_{\mathcal{F}_{b, f i n}^{+}}$Spec, if

1. bound $_{b}(\operatorname{Impl}) \subseteq \operatorname{bound}_{b}($ Spec $)$ and 
2. $\forall(w, X, Y) \in \mathcal{F}_{b, f i n}^{+}($Impl $): \exists x \in\{\varepsilon\} \cup \downarrow X \cup \downarrow Y:\left(w x, x^{-1} X, x^{-1} Y\right) \in$ $\mathcal{F}_{b, f i n}^{+}($Spec $)$.

We say $(w, X, Y)$ is dominated by $\left(w x, x^{-1} X, x^{-1} Y\right)$. For two interface-equivalent open nets Impl and Spec, we define Impl $\sqsubseteq_{\mathcal{F}_{b, f i n}^{+}}$Spec, if env(Impl) $\sqsubseteq_{\mathcal{F}_{b, \text { fin }}^{+}}$ env(Spec).

If two open nets are in the $\mathcal{F}_{b, f i n}^{+}$-refinement relation, then this implies inclusion of their bounded languages, stop-traces, and dead-traces.

Lemma 7.14. For two labeled nets Impl and Spec, we have

1. Impl $\sqsubseteq_{\mathcal{F}_{b, f i n}^{+}}$Spec implies $L_{b}(\operatorname{Impl}) \subseteq L_{b}($ Spec $)$.

2. Impl $\sqsubseteq_{\mathcal{F}_{b, \text { fin }}^{+}}^{+}$Spec implies stop $($Impl $) \subseteq \operatorname{stop}_{b}($ Spec $)$.

3. Impl $\sqsubseteq_{\mathcal{F}_{b, f i n}^{+}}^{+}$Spec implies $\operatorname{dead}_{b}(\operatorname{Impl}) \subseteq \operatorname{dead}_{b}($ Spec $)$.

Proof. (1) Let $w \in L_{b}\left(\right.$ Impl). Then $(w, \emptyset, \emptyset) \in \mathcal{F}_{b, f i n}^{+}($Impl $)$by Definition 7.12 and $(w, \emptyset, \emptyset) \in \mathcal{F}_{b, f i n}^{+}($Spec $)$by Definition 7.13 , which immediately implies $w \in$ $L_{b}$ (Spec) by Definition 7.12

(2) Let $w \in \operatorname{stop}_{b}(\operatorname{Impl})$. Then we use the proof of (the implication of) Theorem 4.17 by replacing stop by stop $b$ to conclude that $w \in \operatorname{stop}_{b}($ Spec $)$.

(3) Similar argumentation as for (2).

Example 7.4. In Example 7.2 we showed for the open nets $D$ and $D^{\prime}$ with the empty set of final markings that $L_{b}$-inclusion does not hold. Thus, with Lemma 7.14(1) we conclude that $D^{\prime}$ does not $\mathcal{F}_{b, f i n}^{+}$-refine $D$.

Again, the composition of two open nets $N_{1}$ and $N_{2}$ has the same $b$-bounded $\mathcal{F}_{f i n}^{+}$-semantics as the parallel composition of the two environments, env $\left(N_{1}\right) \Uparrow$ $\operatorname{env}\left(N_{2}\right)$.

Lemma 7.15. For composable open nets $N_{1}$ and $N_{2}$, we have

$$
\mathcal{F}_{b, f i n}^{+}\left(N_{1} \oplus N_{2}\right)=\mathcal{F}_{b, f i n}^{+}\left(e n v\left(N_{1}\right) \Uparrow e n v\left(N_{2}\right)\right) .
$$

Proof. Follows directly from Lemma 2.12 If one net has a bound $b_{b}$-violator $w$ due to marking $m$, then the other net can reach an agreeing marking $m^{\prime}$ with trace $w$; thus $w$ is also a bound $_{b}$-violator for the other net. Likewise, by applying the same argumentation as in the proof of Lemma 4.10. we conclude that if one net has a $b$-bounded fintree failure $(w, X, Y)$ so does the other net.

We shall show that $\mathcal{F}_{b, f i n}^{+}$-refinement is a precongruence on open nets for $\oplus$, thereby using the precongruence results for $\mathcal{F}_{\text {fin }}^{+}$-refinement and $b$-accordance. We characterize the $b$-bounded $\mathcal{F}_{\text {fin }}^{+}$-semantics for labeled net composition and hiding and finally combine these results to determine the $b$-bounded $\mathcal{F}_{f i n}^{+}$-semantics for open net composition. First, we consider the $b$-bounded $\mathcal{F}_{\text {fin }}^{+}$-semantics for the composition of two labeled nets. Here and below, we use $\pi_{1}(w)$ and $\pi_{2}(w)$ to denote $\left.w\right|_{\Sigma_{1}}$ and $\left.w\right|_{\Sigma_{2}}$ for labeled nets $N_{1}$ and $N_{2}$ with alphabets $\Sigma_{1}$ and $\Sigma_{2}$. 
Lemma $7.16\left(\mathcal{F}_{b, f i n}^{+}\right.$-semantics for labeled net composition). For two composable labeled nets $N_{1}$ and $N_{2}$, we have

$$
\begin{aligned}
\mathcal{F}_{b, f i n}^{+}\left(N_{1} \| N_{2}\right)= & \left\{\left(w, X_{1} \cup X_{2}, Y_{1} \cup Y_{2}\right) \mid \begin{array}{l}
\left(\pi_{1}(w), \pi_{1}\left(X_{1}\right), \pi_{1}\left(Y_{1}\right)\right) \in \mathcal{F}_{b, f i n}^{+}\left(N_{1}\right), \\
\left.\left(\pi_{2}(w), \pi_{2}\left(X_{2}\right), \pi_{2}\left(Y_{2}\right)\right) \in \mathcal{F}_{b, f i n}^{+}\left(N_{2}\right)\right\}
\end{array}\right. \\
\cup \text { finbound }_{b}\left(N_{1} \| N_{2}\right) . &
\end{aligned}
$$

Proof. We write $E$ for $N_{1} \| N_{2}$.

$\subseteq$ : Let $(w, X, Y) \in \mathcal{F}_{b, f i n}^{+}(E)$. If $w$ is not a bound -violator, then $(w, X, Y) \in$ $\mathcal{F}_{\text {fin }}^{+}(E)$ by Definition 7.12 and we conclude with Lemma 4.11 and Definition 7.12 that it is contained in the first set on the right hand side. If $w$ is a bound bviolator $^{\text {-vat }}$ of $E$, then $(w, X, Y) \in$ finbound $_{b}\left(N_{1} \| N_{2}\right)$ by Definition 7.12 .

$\supseteq$ : Let $i=1,2$. If both $\left(\pi_{i}(w), \pi_{i}\left(X_{i}\right), \pi_{i}\left(Y_{i}\right)\right) \in \mathcal{F}_{\text {fin }}^{+}\left(N_{i}\right)$, then $\left(w, X_{1} \cup\right.$ $\left.X_{2}, Y_{1} \cup Y_{2}\right) \in \mathcal{F}_{\text {fin }}^{+}(E)$ by Lemma 4.11 and $\mathcal{F}_{\text {fin }}^{+}(E) \subseteq \mathcal{F}_{b, f i n}^{+}(E)$. Assume now that at least one $b$-bounded fintree farlure $\left(\pi_{i}(w), \pi_{i}\left(X_{i}\right), \pi_{i}\left(Y_{i}\right)\right)$ is not contained in the respective $\mathcal{F}_{f i n}^{+}$-semantics. Then trace $\pi_{i}(w)$ is a bound $d_{b}$-violator by Definition 7.12 and so is $w$ by Proposition $5.3(1)$, because $\pi_{3-i}(w) \in L_{b}\left(N_{3-i}\right)$ as argued in the proof of Lemma 7.14 (1). Thus, $\left(w, X_{1} \cup X_{2}, Y_{1} \cup Y_{2}\right) \in$ finbound $_{b}(E) \subseteq$ $\mathcal{F}_{b, f i n}^{+}(E)$ due to Definition 7.12

In the next lemma, we consider a labeled net $N / A, A \subseteq \Sigma$ and we use $\phi(w)$ to denote $\left.w\right|_{\Sigma \backslash A}$. We canonically extend the notion of $\phi(w)$ pointwise to sets of traces.

Lemma 7.17. For any labeled net $N$ and any label set $A \subseteq \Sigma_{N}^{*}$, we have

$$
\mathcal{F}_{b, f i n}^{+}(N / A)=\left\{(\phi(w), X, Y) \mid\left(w, \phi^{-1}(X), \phi^{-1}(Y)\right) \in \mathcal{F}_{b, f i n}^{+}(N)\right\} .
$$

Proof. Follows from Lemma 4.12 .

The next proposition characterizes the $b$-bounded $\mathcal{F}_{\text {fin }}^{+}$-semantics for open net composition. In the proofs of the following two results, we will denote the first set on the right-hand side in Lemma 7.16 by $\mathcal{F} 1_{b, f i n}^{+}\left(N_{1}, N_{2}\right)$.

Proposition $7.18\left(\mathcal{F}_{b, f i n}^{+}\right.$-semantics for open net composition). For two composable open nets $N_{1}$ and $N_{2}$, we have

$$
\begin{gathered}
\mathcal{F}_{b, \text { fin }}^{+}\left(N_{1} \oplus N_{2}\right)=\quad\left\{(w, X, Y) \mid \exists\left(w_{1}, X_{1}, Y_{1}\right) \in \mathcal{F}_{b, \text { fin }}^{+}\left(N_{1}\right),\left(w_{2}, X_{2}, Y_{2}\right) \in \mathcal{F}_{b, \text { fin }}^{+}\left(N_{2}\right):\right. \\
w \in w_{1} \Uparrow w_{2} \wedge \forall x \in X, y \in Y: \\
\left(x \in x_{1} \Uparrow x_{2} \text { implies } x_{1} \in X_{1} \vee x_{2} \in X_{2}\right) \\
\left.\wedge\left(y \in y_{1} \Uparrow y_{2} \text { implies } y_{1} \in Y_{1} \vee y_{2} \in Y_{2}\right)\right\} \\
\cup \text { finbound }_{b}\left(N_{1} \oplus N_{2}\right) .
\end{gathered}
$$

Proof. According to Lemma 7.15 we can consider $\mathcal{F}_{b, f i n}^{+}\left(\operatorname{env}\left(N_{1}\right) \Uparrow \operatorname{env}\left(N_{2}\right)\right)$ instead of $\mathcal{F}_{b, f i n}^{+}\left(N_{1} \oplus N_{2}\right)$. Because $\Uparrow$ is $\|$ followed by hiding, we can determine this set by applying hiding (according to Lemma 7.17) to the right-hand side of Lemma 7.16. As a result, $\mathcal{F} 1_{b}^{+}\left(N_{1}, N_{2}\right)$ turns into the first set in the present proposition, just as Proposition 4.13 results from Lemma 4.11 with Lemma 4.12 . More easily, finbound $\left(N_{1} \| N_{2}\right)$ is analogously translated into finbound $b\left(N_{1} \oplus N_{2}\right)$ according to Proposition 5.3.(1) and 5.3(7). 
Lemma 7.19. $\mathcal{F}_{b}^{+}$-refinement is a precongruence on labeled nets for composition operator $\|$.

Proof. For this proof, we will use that the following four saturation conditions, which hold for the $\mathcal{F}_{f i n}^{+}$-semantics (see Lemma 4.14), also hold for the $b$-bounded $\mathcal{F}_{\text {fin }}^{+}$-semantics:

SAT1: $(w, X, Y) \in \mathcal{F}_{b, f i n}^{+}(N), X^{\prime} \subseteq X, Y^{\prime} \subseteq Y$ implies $\left(w, X^{\prime}, Y^{\prime}\right) \in \mathcal{F}_{b, f i n}^{+}(N)$

SAT2: $(w, X, Y) \in \mathcal{F}_{b, \text { fin }}^{+}(N) \wedge \forall z \in Z:\left(w z, z^{-1} X, z^{-1} Y\right) \notin \mathcal{F}_{b, f i n}^{+}(N)$ implies $(w, X \cup Z, Y \cup Z) \in \mathcal{F}_{b, f i n}^{+}(N)$

SAT3: $(w, X, Y) \in \mathcal{F}_{b, f i n}^{+}(N)$ implies $(w, \uparrow X, Y) \in \mathcal{F}_{b, f i n}^{+}(N)$

SAT4: $(w, X, Y) \in \mathcal{F}_{b, f i n}^{+}(N)$ implies $(w, X, X \cup Y) \in \mathcal{F}_{b, f i n}^{+}(N)$

To see these conditions, consider first some $(w, X, Y) \in \mathcal{F}_{\text {fin }}^{+}(N) \subseteq \mathcal{F}_{b, f i n}^{+}(N)$. Then, SAT1 - SAT4 follow directly from Lemma 4.14 . Now, consider a $b$-bounded fintree failure $(w, X, Y)$ with $w \in$ bound $_{b}(N)$; here, all four conditions are immediate because $\left(w, X^{\prime}, Y^{\prime}\right) \in \mathcal{F}_{b, f i n}^{+}(N)$ for any $X^{\prime} \in \mathcal{P}\left((I \uplus O)^{+}\right)$and $Y^{\prime} \in \mathcal{P}\left((I \uplus O)^{*}\right)$.

The precongruence result for $\mathcal{F}_{f i n}^{+}$-refinement holds for general sets of fintree failures (see Remark 4.2 below Lemma 4.14). We make use of this, although this defining equation does not match Lemma 7.16 , but just gives $\mathcal{F} 1_{b, f i n}^{+}\left(N_{1}, N_{2}\right)$.

Now let Impl $\sqsubseteq_{\mathcal{F}_{b, f i n}^{+}}$Spec and $C$ be a composable labeled net for Impl and Spec. We have to check the two items of Definition 6.15 in order to prove that $\operatorname{Impl} \| C \sqsubseteq_{\mathcal{F}_{b, \text { fin }}^{+}}$Spec $\| C$.

The first item follows from Proposition 5.3(1) (which holds for labeled nets in general) because our assumption implies bound $\left(\operatorname{Impl}_{b} \subseteq\right.$ bound $_{b}($ Spec $)$ as well as - due to Lemma 7.14 (1)- $L_{b}($ Impl $) \subseteq L_{b}($ Spec $)$.

For the second item, we first consider some $(w, X, Y) \in \mathcal{F} 1_{b, f i n}^{+}(\operatorname{Impl}, C)$. We observe that, due to Definition $7.13(2), \mathcal{F}_{b, f i n}^{+}($Impl $)$is related to $\mathcal{F}_{b, f i n}^{+}($Spec $)$in the sense of $\mathcal{F}_{\text {fin }}^{+}$-refinement. Thus, according to Lemma 4.14. $\exists x \in\{\varepsilon\} \cup \downarrow X \cup$ $\downarrow Y:\left(w x, x^{-1} X, x^{-1} Y\right) \in \mathcal{F} 1_{b, \text { fin }}^{+}($Spec,$C) \subseteq \mathcal{F}_{b, \text { fin }}^{+}($Spec $\| C)$. Second, we consider some $(w, X, Y) \in$ finbound $_{b}(\operatorname{Impl} \| C)$. This time, due to bound $(\operatorname{Impl} \| C) \subseteq$ bound $_{b}(\operatorname{Spec} \| C)$, we even have $(w, X, Y) \in$ finbound $_{b}(\operatorname{Spec} \| C) \subseteq \mathcal{F}_{b, \text { fin }}^{+}(\operatorname{Spec} \| C)$; that is, Definition $7.13(2)$ is satisfied taking $x=\varepsilon$.

Lemma 7.20. $\mathcal{F}_{b, f i n}^{+}$-refinement is a precongruence on labeled nets for hiding.

Proof. Let Impl and Spec be labeled nets with $\operatorname{Impl} \sqsubseteq_{\mathcal{F}_{b, f i n}^{+}} S p e c$, and $A \subseteq \Sigma^{*}$. Then Lemma 7.17(1) directly implies Definition 7.13(1) for Impl/A and Spec/A. Furthermore, the characterization in Lemma $7.17(2)$ corresponds to the defining equation for hiding in Lemma 4.15, so Definition 7.13(2) is inherited from the precongruence result in Lemma 4.15 for $\mathcal{F}_{\text {fin }}^{+}$-refinement and hiding.

Now we directly get the first main result of this section. 
Theorem 7.21 (precongruence). $\mathcal{F}_{b, f i n}^{+}$-refinement is a precongruence on open nets for composition operator $\oplus$.

Proof. This is now completely analogous to the proof of Theorem 4.16, except that here the semantics associates two sets with a net and we use Lemma 7.19 and 7.20 on the basis of Lemma 4.14 and Lemma 4.15 .

As for $\sqsubseteq_{b r, a c c}^{c}$ in Lemma 6.13 , we show that $\sqsubseteq_{b f r, a c c}^{c}$ implies $\sqsubseteq_{b, a c c}$.

Lemma $7.22\left(\sqsubseteq_{b f r, a c c}^{c}\right.$ implies $\left.\sqsubseteq_{b, a c c}\right)$. For two open nets Impl and Spec, we have

$$
\text { Impl } \sqsubseteq_{b f r, a c c}^{c} \text { Spec implies Impl } \sqsubseteq_{b, a c c} \text { Spec. }
$$

Proof. Use the proof of Lemma 6.13 while assuming the set of final markings to be the empty set for all open nets.

Next, we prove our second main result of this section: $\mathcal{F}_{b, f i n}^{+}$-refinement coincides with the coarsest precongruence that is contained in the $b f r$-accordance relation.

Theorem 7.23 (precongruence and $\mathcal{F}_{b, f i n}^{+}$-refinement coincide). For two interface-equivalent open nets Impl and Spec, we have

$$
\text { Impl } \sqsubseteq_{b f r, a c c}^{c} \text { Spec iff Impl } \sqsubseteq_{\mathcal{F}_{b, f i n}^{+}} \text {Spec. }
$$

Proof. $\Rightarrow$ : By Lemma 7.22, $\sqsubseteq_{b f r, a c c}^{c}$ implies $\sqsubseteq_{b, a c c}$ from which we conclude bound $_{b}$-inclusion. It remains to show $\mathcal{F}_{b, \text { fin }}^{+}$-inclusion. Let $(w, X, Y) \in \mathcal{F}_{b, f i n}^{+}(\operatorname{Impl})$ with $w \notin$ bound $_{b}(\operatorname{Impl})$. Otherwise, $w \in$ bound $_{b}(\operatorname{Impl}) \subseteq$ bound $_{b}($ Spec $)$ by bound $_{b}$-inclusion, and $(w, X, Y) \in \mathcal{F}_{b, \text { fin }}^{+}($Spec $)$by Definition 7.12 . We use net $N$ in Fig. 6 as in the proof of Theorem 4.17. Following the argumentation in the proof of Theorem 4.17, we have that if an open net $C$ is not a $b f r$-controller of $\operatorname{Impl} \oplus N$ so it is not a $b f r$-controller of Spec $\oplus N$. We distinguish three cases: If $w \in$ bound $_{b}($ Spec $)$, then $(w, X, Y) \in \mathcal{F}_{b, \text { fin }}^{+}($Spec $)$by Definition 7.12 If $w u \in$ bound $_{b}($ Spec $)$ with $u \in \downarrow X \cup \downarrow Y$, then $\left(w u, u^{-1} X, u^{-1} Y\right) \in \mathcal{F}_{b, \text { fin }}^{+}$(Spec) by Definition 7.12. Otherwise, we use the argumentation in the proof of Theorem 4.17 to conclude that $(w, X, Y) \in \mathcal{F}_{b, f i n}^{+}($Spec $)$.

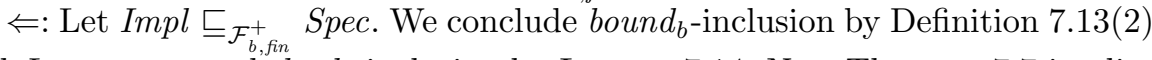
and $L_{b^{-}}$, stop $b^{-}$, and $d e a d_{b^{-}}$-inclusion by Lemma 7.14. Now Theorem 7.7 implies Impl $\sqsubseteq_{b f r, a c c} S p e c$, and this, in turn, also shows that Impl $\sqsubseteq_{\mathcal{F}_{b, f i n}^{+}}$Spec implies Impl $\sqsubseteq_{b f r, a c c}^{c}$ Spec with Theorem 7.21 and the definition of $\sqsubseteq_{b f r, a c c}^{c}$.

Example 7.5. In Example 7.2, we showed that $D^{\prime} b f r$-accords with $D$ (with the empty set of final markings) but $D^{\prime}$ does not $\mathcal{F}_{b, f i n}^{+}$-refine $D$ by Example 7.4. Consequently, $D^{\prime} \sqsubseteq_{b f r, a c c}^{c} D$ does not hold. 


\subsection{Decidability of $\mathcal{F}_{b, f i n}^{+}$-refinement}

In this section, we show that checking $\mathcal{F}_{b, f i n}^{+}$-refinement is decidable. Checking $\mathcal{F}_{b, f i n}^{+}$-refinement entails checking both items of Definition 7.13 The first item of Definition 7.13 that is, checking bound $_{b}$-inclusion - is decidable; see Sect. 6.4. To decide refinement of the $b$-bounded fintree failures - that is, the second item of Definition 7.13 - we apply a similar proof strategy as in the case of $\mathcal{F}_{b}^{+}$refinement. First, we show that the construction of Rensink and Vogler 29, Theorem 61] for deciding $\mathcal{F}^{+}$-refinement of two finite LTS can be generalized to decide $\mathcal{F}_{f i n}^{+}$-refinement. Then we present an encoding of the $b$-bounded fintree failures of two open nets as finite LTS on which we decide $\mathcal{F}_{\text {fin }}^{+}$-refinement. That way, we can conclude decidability of $\mathcal{F}_{b, f i n}^{+}$-refinement.

Deciding $\mathcal{F}_{\text {fin }}^{+}$-refinement for finite LTS We assume two finite transition systems Impl and Spec with initial states $p_{0}$ and $q_{0}$ such that $L(\operatorname{Impl}) \subseteq L($ Spec $)$. Checking language inclusion is known to be decidable. We denote the transition relations with $\rightarrow_{\text {Impl }}$ and $\Rightarrow_{\text {Impl }}$.

We can view each finite transition system as a finite automaton where all states are accepting. For an automaton $A$ with some state $s$ (we write $s \in A$ ), $L_{A}(s)$ denotes the language of the automaton if we change the initial state to $s$. Observe that the arc label $\tau$ corresponds to the empty word in automata theory. We consider an automaton $A$ with two types of accepting states, called 1-accepting and 2-accepting. With $L^{i}(A)$ we denote the language of $A$ when $i$ accepting states are considered to be accepting states, for $i=1,2$. For the sake of notation, $L(A)$ denotes $L^{1}(A) \cup L^{2}(A)$. We call a state productive, if it lies on a path from the initial state to some accepting state - that is, if it is used by the automaton when accepting a word.

As a first step, we extend the automaton Impl to an automaton of automata $A A$ by adding a family of pairs of deterministic automata $\left(A_{p}^{1}, A_{p}^{2}\right), p \in I m p l$, such that for every $p \in I m p l$ the language of $A_{p}^{1}$ is the set $\Sigma^{*} \backslash L_{I m p l}(p)$ of traces that Impl cannot perform from $p$, and the language of $A_{p}^{2}$ is the set $\Sigma^{*} \backslash L_{f i n}(p)$ with $L_{f i n}(p)=\left\{w \mid p \stackrel{w}{\rightarrow} p^{\prime} \wedge p^{\prime} \in \Omega_{\text {Impl }}\right\}$. The following holds:

$$
(w, X, Y) \in \mathcal{F}_{b, f i n}^{+}(\operatorname{Impl}) \text { iff } \exists p_{0} \stackrel{w}{\Longrightarrow} A A p: X \subseteq L\left(A_{p}^{1}\right) \wedge Y \subseteq L\left(A_{p}^{2}\right) .
$$

Thus the automata $A_{p}$ represent some $b$-bounded fintree failures $(w, X, Y) \in$ $\mathcal{F}_{b, f i n}^{+}(\operatorname{Impl})$ in the sense that there is a $p \in \operatorname{Impl}$ with $p_{0} \stackrel{w}{\Longrightarrow} A A p$ and $X \subseteq$ $L\left(A_{p}^{1}\right)$ and $Y \subseteq L\left(A_{p}^{2}\right)$; in particular, they represent all maximal $b$-bounded fintree failures - that is, all those $(w, X, Y) \in \mathcal{F}_{b, f i n}^{+}(\operatorname{Impl})$ with maximal $X$ and $Y$. Note that in a finite transition system $I m p l$, there exists for each $\left(v, X^{\prime}, Y^{\prime}\right) \in$ $\mathcal{F}_{b, f i n}^{+}(\operatorname{Impl})$ a maximal $(v, X, Y) \in \mathcal{F}_{b, f i n}^{+}(\operatorname{Impl})$ with $X^{\prime} \subseteq X$ and $Y^{\prime} \subseteq Y$.

Similar, we construct an automaton of pairs of automata for Spec, but this time, we additionally make Spec deterministic more or less by the usual powerset construction. This results in a deterministic automaton of pairs of automata $B B$, 
which is a deterministic automaton extended with a family $B B_{Q}, Q \in B B$. For each state $Q$ (being a set of states of Spec), $B B_{Q}$ is a pair of deterministic automata $\left(B_{q}^{1}, B_{q}^{2}\right), q \in Q$ with $L\left(B_{q}^{1}\right) \subseteq \Sigma \backslash L_{\text {Spec }}(q)$ and $L\left(B_{q}^{2}\right) \subseteq \Sigma \backslash L_{\text {fin,Spec }}(q)$.

More in detail, the automaton part of $B B$ is defined as follows: The initial state of $B B$ is $Q_{0}=\left\{q \mid q_{0} \stackrel{\tau^{*}}{\longrightarrow}\right.$ Spec $\left.q\right\}$; the transition relation is defined by $Q \stackrel{a}{\rightarrow}_{B B} Q^{\prime}$ if $Q^{\prime}=\left\{q^{\prime} \mid \exists q \in Q: q \stackrel{a}{\rightarrow}_{\text {Spec }}{\stackrel{\tau^{*}}{\rightarrow}}_{\text {Spec }} q^{\prime}\right\}$. We restrict $B B$ to the nonempty states reachable from $Q_{0}$ and let each state of $B B$ be accepting. As a consequence, all states of $B B$ are productive and $L(B B) \subseteq L($ Spec $)$.

This way, $Q_{0} \stackrel{w}{\Longrightarrow} B B Q$ iff $Q=\left\{q \mid q_{0} \stackrel{w}{\Longrightarrow}\right.$ Spec $\left.q\right\}$ for all $w \in \Sigma^{*}$ and

$$
\begin{aligned}
& (w, X, Y) \in \mathcal{F}_{b, f i n}^{+}(\text {Spec }) \text { iff } \\
& \exists Q_{0} \stackrel{w}{\Longrightarrow} B B Q,\left(B^{1}, B^{2}\right) \in B B_{Q}: X \subseteq L\left(B^{1}\right) \wedge Y \subseteq L\left(B^{2}\right) .
\end{aligned}
$$

First, we construct the following partial product automaton $S$, which can also be seen as the minimal simulation from $A A$ to $B B$ :

$-\left(p_{0}, Q_{0}\right) \in S$ is the initial state of $S$ and all states are accepting.

- If $(p, Q) \in S, a \in \Sigma$ and $p \stackrel{a}{\rightarrow}_{A A} p^{\prime}$, then by language inclusion and definition of $B B$, there is a unique $Q^{\prime} \in B B$ such that $Q \stackrel{a}{\rightarrow}{ }_{B B} Q^{\prime}$; we add $\left(p^{\prime}, Q^{\prime}\right)$ and the transition $(p, Q) \stackrel{a}{\rightarrow}\left(p^{\prime}, Q^{\prime}\right)$ to $S$.

- If $(p, Q) \in S$ and $p \stackrel{\tau}{\rightarrow} A A p^{\prime}$, then we add $\left(p^{\prime}, Q\right)$ and the transition $(p, Q) \stackrel{\tau}{\rightarrow}$ $\left(p^{\prime}, Q\right)$ to $S$ (recall that $B B$ has no $\tau$ labeled arcs).

Checking $\mathcal{F}_{b, f i n}^{+}$-refinement entails checking whether for all $(w, X, Y) \in \mathcal{F}_{b, f i n}^{+}(\operatorname{Impl})$ with $X \cup Y \neq \emptyset$, we have $\left(w u, u^{-1} X, u^{-1} Y\right) \in \mathcal{F}_{b, f i n}^{+}($Spec $)$for some $u \in$ $\downarrow(X \cup Y)$. Recall that by language inclusion we do not have to check triples $(w, \emptyset, \emptyset)$. We have to check for each $(p, Q) \in S$ and each pair $(X, Y)$ with $X \subseteq L\left(A_{p}^{1}\right), Y \subseteq L\left(A_{p}^{2}\right)$, and $X \cup Y \neq \emptyset$ that

$$
\begin{aligned}
& \exists u \in \downarrow(X \cup Y), Q^{\prime} \in B B,\left(B^{1}, B^{2}\right) \in B B_{Q^{\prime}}: \\
& Q \stackrel{u}{\Longrightarrow}{ }_{B B} Q^{\prime} \wedge u^{-1} X \subseteq L\left(B^{1}\right), u^{-1} Y \subseteq L\left(B^{2}\right)
\end{aligned}
$$

Let us fix $(p, Q)$; we now show how to check (1) for all suitable $(X, Y)$. This means that we have to compare runs in $A_{p}^{1}$ or $A_{p}^{2}(u$ in (1) with runs of $B B$. To do this, we construct another (partial) product automaton $P$, similar to the previous one, but this time between the automata $A_{p}^{1}, A_{p}^{2}$ (whose initial states we also denote by $p$ ) and $B B$ where the initial state is changed to $Q$. Another difference with the case above is that, this time, we do not necessarily have $L\left(A_{p}^{1}\right) \cup L\left(A_{p}^{2}\right) \subseteq L_{B B}(Q)$ — that is, $B B$ might not be able to simulate both, $A_{p}^{1}$ and $A_{p}^{2}$-but still we want to represent all of $L\left(A_{p}^{1}\right) \cup L\left(A_{p}^{2}\right)$ in order to check the inclusion in (1). Therefore, $P$ is constructed as follows (here $*$ is a dummy element, not appearing anywhere else):

$-(p, p, Q) \in P$ is the initial state; 
- $\left(p_{1}, p_{2}, Q^{\prime}\right) \in P$ and $p_{1} \stackrel{a}{\rightarrow}_{A_{p}^{1}} p_{1}^{\prime}$ or $p_{2} \stackrel{a}{\rightarrow}_{A_{p}^{2}} p_{2}^{\prime}$ (implying $p_{1} \neq * \neq p_{1}^{\prime}$ or $\left.p_{2} \neq * \neq p_{2}^{\prime}\right)$, we add state $\left(p_{1}^{\prime \prime}, p_{2}^{\prime \prime}, Q^{\prime \prime}\right)$ and the transition $\left(p_{1}, p_{2}, Q^{\prime}\right) \stackrel{a}{\rightarrow}$ $\left(p_{1}^{\prime \prime}, p_{2}^{\prime \prime}, Q^{\prime \prime}\right)$ where

- $p_{i}^{\prime \prime}$ is $p_{i}^{\prime}$ if $p_{i} \stackrel{a}{\rightarrow} A_{p}^{i} p_{i}^{\prime}$ and $*$ otherwise (in particular if $p_{i}=*$ ) for $i=1,2$

- $Q^{\prime \prime}$ satisfies $Q^{\prime} \stackrel{a}{\rightarrow}_{B B} Q^{\prime \prime}$ (in particular, $Q^{\prime}=*$ or is $*$ otherwise

- $\left(p_{1}, p_{2}, Q^{\prime}\right)$ is $i$-accepting if $p_{i}$ is accepting in $A_{p}^{i}, i=1,2$.

Because the $A_{p}^{i}$ and $B B$ are deterministic, $P$ is also deterministic, and we have $L^{i}(P) \subseteq L\left(A_{p}^{i}\right)$ for $i=1,2$ by construction. We will call $R$ a productive subautomaton of $P$, if $R$ is obtained from $P$ by restricting all components (in particular also the accepting states) to a subset $M$ of the state set such that each state of $R$ is productive in $h^{5}$. We will show that (1) is satisfied for all suitable $(X, Y)$ if and only if for each productive subautomaton $R$ of $P$

$$
\begin{aligned}
\exists\left(p_{1}, p_{2}, Q^{\prime}\right) \in R, Q^{\prime} \in B B,\left(B^{1}, B^{2}\right) \in B B_{Q^{\prime}}: \\
L_{R}^{i}\left(p_{1}, p_{2}, Q^{\prime}\right) \subseteq L\left(B^{i}\right), \text { for } i=1,2
\end{aligned}
$$

Because the latter is clearly decidable, it then follows that $\mathcal{F}_{b, f i n}^{+}$-refinement is decidable. Note that $Q^{\prime} \in B B$ in (2) is equivalent to $Q^{\prime} \neq *$.

So assume (1) is satisfied for all suitable $(X, Y)$. If $R$ is a productive subautomaton, then $L^{1}(R) \subseteq L^{1}(P) \wedge L^{2}(R) \subseteq L^{2}(P) \wedge L^{1}(R) \cup L^{2}(R) \neq \emptyset$. Hence, due to (1) and the construction of $P, \exists u \in \downarrow L^{1}(R) \cup \downarrow L^{2}(R), Q^{\prime} \in$ $B B,\left(B^{1}, B^{2}\right) \in B B_{Q^{\prime}}: Q \stackrel{u}{\Longrightarrow} B B Q^{\prime}$ and $u^{-1} L^{i}(R) \subseteq L\left(B^{i}\right)$, for $i=1,2$. Then $(p, p, Q) \stackrel{u}{\Longrightarrow} R\left(p_{1}, p_{2}, Q^{\prime}\right)$ for some $p_{1}, p_{2}$; because $R$ is deterministic, $\left(p_{1}, p_{2}, Q^{\prime}\right)$ is uniquely determined by $u$, and therefore $u^{-1} L^{i}(R)=L_{R}^{i}\left(\left(p_{1}, p_{2}, Q^{\prime}\right)\right)$. Thus, $\left(p_{1}, p_{2}, Q^{\prime}\right)$ and $\left(B^{1}, B^{2}\right)$ are the state and automaton pair whose existence is asserted in (2).

Vice versa, assume that (2) holds for each productive subautomaton $R$ and take some $X \subseteq L\left(A_{p}^{1}\right)$ and $Y \subseteq L\left(A_{p}^{2}\right)$ with $X \cup Y \neq \emptyset$. The set of states that are needed in $P$ to accept the words of $X \cup Y$ (recall the construction of $P$ ) defines a productive subautomaton $R$ with $X \subseteq L^{1}(R)$ and $Y \subseteq L^{2}(R)$. Take $\left(p_{1}, p_{2}, Q^{\prime}\right) \in$ $R$ and $\left(B^{1}, B^{2}\right) \in B B_{Q^{\prime}}$ that satisfies (2). Then there is some $u \in \downarrow X \cup \downarrow Y$ with $(p, p, Q) \stackrel{u}{\Longrightarrow} P\left(p_{1}, p_{2}, Q^{\prime}\right)$ by choice of $R$ and $Q \stackrel{u}{\Longrightarrow}{ }_{B B} Q^{\prime}$ by construction of $P$ and because $Q^{\prime} \in B B$. Now $u^{-1} X \subseteq u^{-1} L^{1}(R)=L_{R}^{1}\left(\left(p_{1}, p_{2}, Q^{\prime}\right)\right)$ and $u^{-1} Y \subseteq u^{-1} L^{2}(R)=L_{R}^{1}\left(\left(p_{1}, p_{2}, Q^{\prime}\right)\right)$ by determinism of $R$, and we can conclude that $u^{-1} X \subseteq L\left(B^{1}\right)$ and $u^{-1} Y \subseteq L\left(B^{2}\right)$.

Therefore, we have shown:

Proposition 7.24. Checking $\mathcal{F}_{\text {fin }}^{+}$-refinement for two finite LTS is decidable.

\footnotetext{
${ }^{5}$ Recall that a state of an automaton is productive if it lies on a path from the initial to some accepting state.
} 


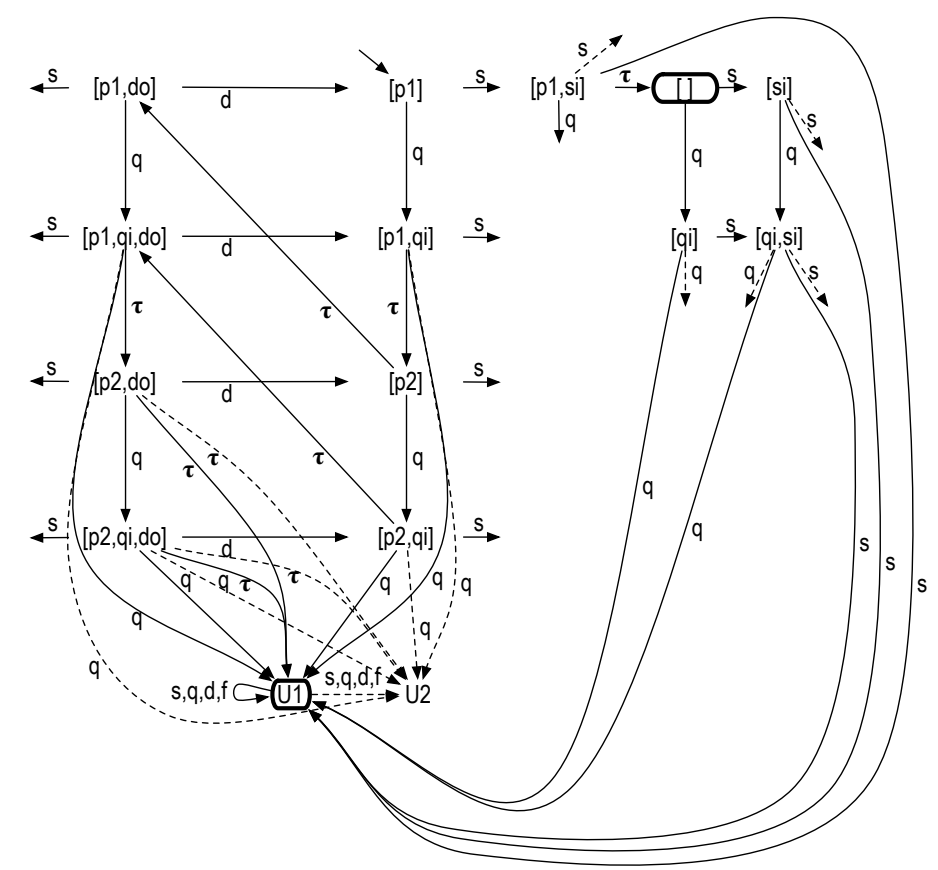

Fig. 16: Sketch of the finite automaton $U_{12}(D)$ as used in the proof of Theorem 7.28 . The two final states $U_{1}$ and [] are depicted by a thick frame. An arrow without sink sketches a transition, with sketched dashed transitions always leading to state $U_{2}$.

Reducing decision of $\mathcal{F}_{b, \text { fin }}^{+}$-refinement to $\mathcal{F}_{\text {fin }}^{+}$-refinement As we did for $\mathcal{F}_{b}^{+}$-refinement in Sect. 6.4 we prove decidability of $\mathcal{F}_{b, f i n}^{+}$-refinement for arbitrary LTS rather than for reachability graphs of labeled nets. For an LTS $S$, we construct a finite LTS $U_{12}(S)$ (see Sect. 6.4). In this section we consider-in contrast to Sect. 6.4 - LTS with final states and define the set of final states of $U_{12}(S)$ by the final states of $S$ and additionally $U_{1}$.

Example 7.6. Figure 16 sketches the automaton that represents bound $d_{1}(D)$. Parts of the automaton in Fig. 16 that can only be reached with an $s$-labeled transition are not shown. Likewise, the $q$-labeled transition leaving the state $\left[p_{1}, s^{i}\right]$ is not shown. The set of final markings of the open net $D$ is $\{[]\}$; thus, $U_{12}(D)$ has final states [] and $U_{1}$.

The next lemma, gives three observations about $U_{12}(S)$.

Lemma 7.25. Let $S$ be an LTS. Then the following facts hold for $U_{12}(S)$.

1. $w \in$ bound $_{b}(S)$ iff $m_{N} \stackrel{w}{\Longrightarrow} U_{1}$ iff $m_{N} \stackrel{w}{\Longrightarrow} U_{2}$. 
2. $B \mathcal{F}_{b, f i n}^{+}(S) \subseteq \mathcal{F}_{\text {fin }}^{+}\left(U_{12}(S)\right)$ (i.e., $U_{2}$ can refuse all $X \subseteq(I \cup O)^{+}$and finrefuse all $\left.Y \subseteq(I \cup O)^{*}\right)$

3. $\mathcal{F}_{\text {fin }}^{+}\left(U_{12}(S)\right) \subseteq \mathcal{F}_{b, f i n}^{+}(S)$ and $(w, X, Y) \in \mathcal{F}_{b, f i n}^{+}(S) \backslash \mathcal{F}_{\text {fin }}^{+}\left(U_{12}(S)\right)$ implies $\exists u \in \downarrow(X \cup Y) \backslash X$ such that $w u \in$ bound $_{b}(S)$

Proof. (1) follows immediately from the definition of $U_{12}(S)$, and (2) is an implication of (1). (3) The sets agree on those $(w, X, Y)$ with $w \in \operatorname{bound}_{b}(S)$ by (2). So consider $w \notin$ bound $_{b}(S)$. If $(w, X, Y) \in \mathcal{F}_{\text {fin }}^{+}\left(U_{12}(S)\right)$ due to $m_{S} \stackrel{w}{\Longrightarrow} m$, then we also have $m_{S} \stackrel{w}{\Longrightarrow} m$ in $S$ with the same run. In $U_{12}(S), m$ could only have more traces (possibly to final states) due to runs using $U_{1}$, so it can only refuse and fin-refuse less. Thus, $(w, X, Y) \in \mathcal{F}_{f i n}^{+}(S)$ and inclusion follows.

If $(w, X, Y) \in \mathcal{F}_{b, f i n}^{+}(S)$ due to $m$, but $(w, X, Y) \notin \mathcal{F}_{\text {fin }}^{+}\left(U_{12}(S)\right)$, then this must be due to a run from $m$ that touches $U_{1}$; assume this happens for the first time after $u \neq \varepsilon$; that is, we have $m_{N} \stackrel{w}{\Longrightarrow} m \stackrel{u}{\Longrightarrow} U_{1} \stackrel{u^{\prime}}{\Longrightarrow}$ with $u u^{\prime} \in X \cup Y$. Thus, $w u \in$ bound $_{b}(S)$ and $u \in \downarrow(X \cup Y)$. Because $m_{N} \stackrel{w}{\Longrightarrow} m \stackrel{u}{\Longrightarrow}$ also in $S$, we further have $u \notin X$.

The following lemma gives another prerequisite for the decidability proof.

Lemma 7.26. For $X, Y \in \mathcal{P}\left(\Sigma^{*}\right)$, let $x \in \downarrow(X \cup Y) \cup\{\varepsilon\}, X^{\prime}=x^{-1} X$ and $Y^{\prime}=x^{-1} Y$. Then

1. $u \in \downarrow\left(X^{\prime} \cup Y^{\prime}\right)$ implies $x u \in \downarrow(X \cup Y)$

2. $u \notin X^{\prime}$ implies $x u \notin X$

Proof. (1) Due to $u u^{\prime} \in X^{\prime} \cup Y^{\prime}$ and $x u u^{\prime} \in X \cup Y$.

(2) Suppose $x u \in Y$. Then $u \in X$ and we have a contradiction.

With the next lemma, we show that deciding $\mathcal{F}_{b, f i n}^{+}$-refinement for two open nets $I m p l$ and $S p e c$ reduces to check $\mathcal{F}_{\text {fin }}^{+}$-refinement of their finite automata $U_{12}($ Spec $)$ and $U_{12}($ Impl $)$.

Lemma 7.27. For two open nets Impl and Spec such that bound $(\operatorname{Impl}) \subseteq$ bound $_{b}($ Spec $)$, we have

$$
\text { Impl } \sqsubseteq_{\mathcal{F}_{b, f i n}^{+}} \text {Spec iff } U_{12}(\text { Impl }) \sqsubseteq_{\mathcal{F}_{\text {fin }}^{+}} U_{12}(\text { Spec }) .
$$

Proof. $\Rightarrow:$ Each $(v, X, Y) \in \mathcal{F}_{\text {fin }}^{+}\left(U_{12}(\operatorname{Impl})\right) \subseteq \mathcal{F}_{b, f i n}^{+}(\operatorname{Impl})$ (by Lemma 7.25(3)) is dominated by some $\left(w, X^{\prime}, Y^{\prime}\right) \in \mathcal{F}_{b, f i n}^{+}($Spec $)$due to some $x \in \downarrow(X \cup Y) \cup\{\varepsilon\}$ i.e., $X^{\prime}=x^{-1} X, Y^{\prime}=x^{-1} Y, w=v x$. If $\left(w, X^{\prime}, Y^{\prime}\right) \in \mathcal{F}_{f i n}^{+}\left(U_{12}(\operatorname{Impl})\right)$, we are done. So assume otherwise and consider $u \in \downarrow\left(X^{\prime} \cup Y^{\prime}\right) \backslash X^{\prime}$ according to Lemma 7.25(3). Then $x u \in \downarrow(X \cup Y) \backslash X$ by Lemma 7.26 and $\left(v x u,(x u)^{-1} X,(x u)^{-1} Y\right) \in \mathcal{F}_{\text {fin }}^{+}\left(U_{12}(\right.$ Spec $\left.)\right)$ because vxu $\in$ bound $_{b}($ Spec $)$ and $\varepsilon \notin(x u)^{-1} X$. Hence, $(v, X, Y)$ is also covered in this case.

$\Leftarrow:$ Each $(w, X, Y) \in \mathcal{F}_{\text {fin }}^{+}\left(U_{12}(\operatorname{Impl})\right)$ is dominated by $\mathcal{F}_{\text {fin }}^{+}\left(U_{12}(\right.$ Spec $\left.)\right) \subseteq$ $\mathcal{F}_{b, f i n}^{+}($Spec $)$by Lemma $7.25(3)$. So consider $(w, X, Y) \in \mathcal{F}_{b, f i n}^{+}(\operatorname{Impl}) \backslash$ 
$\mathcal{F}_{\text {fin }}^{+}\left(U_{12}(\operatorname{Impl})\right)$ and respectively $u \in \downarrow(X \cup Y) \backslash X$ according to Lemma 7.25(3). Then, $\left(w u, u^{-1} X, u^{-1} Y\right) \in \mathcal{F}_{b, f i n}^{+}($Spec $)$because $w u \in$ bound $_{b}(\operatorname{Impl}) \subseteq$ bound $_{b}($ Spec $)$ and $\varepsilon \notin u^{-1} X$. Thus, $(w, X, Y)$ is also dominated in this case.

With Lemma 7.27 and Proposition 7.24, we have shown:

Theorem $7.28\left(\mathcal{F}_{b, f i n}^{+}\right.$-refinement is decidable). For two interface-equivalent open nets Impl and Spec, checking whether Impl $\sqsubseteq_{\mathcal{F}_{b, f i n}^{+}}$Spec is decidable.

\section{Related Work}

The idea of responsiveness for finite state open systems with final states has been coined by Wolf in [37: An open net $N$ is responsive if $\operatorname{inner}(N)$ is $b$-bounded and from every reachable marking we can reach either a final marking or a marking that enables a transition with an output place in its postset [37. In other words, Wolf defines responsiveness for single open nets and considers only such responsive nets; this guarantees a stricter form of our respective responsiveness variant. More generally, we also deal with open nets that are responsive in some open net compositions but not in others. Lohmann and Wolf [20] present a more efficient decision procedure for the responsiveness in [37, but on an automaton model. Responsiveness in [37/20] is mainly motivated by algorithmic considerations for deciding the respective accordance, but without characterizing the latter semantically or studying compositionality. Müller 24] presents an asymmetrical definition from the point of view of one individual open system in a composition. Our notion of responsiveness leads to precongruences, where the related equivalence is similar to P-deadlock equivalence in [33. Desai et al. 11] define responsiveness for bounded message queues. Their notion of responsiveness is stricter than ours because it additionally requires that no message in any channel is ignored forever.

In other work, the term responsiveness refers to different properties: Reed et al. 28 aim at excluding certain deadlocks, whereas responsiveness in our setting refers to the ability to communicate. The works [16]113 consider with the $\pi$-calculus a more expressive model than open nets but in the setting of synchronous communication, whereas we consider asynchronous communication. Moreover, responsiveness in [1]13] and lock-freedom in [16] guarantee that communication over a certain channel is eventually possible. In contrast, our notion of responsiveness requires that communication over some channel is always possible. Kobayashi [16] defines responsiveness over the infinite runs of the system, thereby using a strong fairness for the channel synchronization. Moreover, the language considered in [16] does not support choices. Acciai and Boreale [1] use a type system and reduction rules different from Kobayashi, and they give an example of a responsive process that cannot be expressed in [16. Gamboni and Ravara [13] use a variant of the $\pi$-calculus that is more expressive than the one in [16]: Choices are part of the language and it is also possible to express how many times a communication over a certain channel should take place. In addition to responsiveness in [1] (called activeness in [13]), Gamboni and Ravara 
require that whenever communication takes place over a channel, then the respective processes conform to to a specified protocol. The latter property is called responsiveness in [13. Recently, Padovani [26] takes up lock-freedom from [16]. He defines a behavioral type system using asynchronously communicating session types and considers the progress property. Progress is a stricter notion than bounded final responsiveness as it (like responsiveness in [11]) additionally requires that no message in any channel is ignored forever.

Trace-based semantics like ours (in particular in Sect. 5. where the language is flooded with error traces go back to the work of Dill [12. Errors in 12 ] arise from communication mismatches and are similar to our bound-violators. In contrast to our asynchronous (i.e., buffered) setting, Dill considers a synchronous (i.e., an unbuffered) setting. Dill's semantics can be seen as declarative whereas interface automata, as defined by Alfaro and Henzinger in [10, take up the same ideas on an operational (i.e., automaton) model. Dill's refinement relations are trace inclusions like our characterizations of the four responsiveness preorders. In contrast, refinement of interface automata is characterized by an alternating simulation relation similar to the refinement of modal transition systems [17. Chen et al. 8] (long version in [9]) present more recent work that is based on languages. They consider with quiescent traces a kind of stop traces. The precongruence defined in $[8$ is based on traces but in a synchronous setting. In contrast, our precongruences are variants of the should testing preorder [29]. Moreover, our notion of divergence (i.e., "infinite internal chatter") is different from the one in [9]: If two open systems indefinitely interact with each other, then they are responsive and, hence, we do not treat such trace as problematic, but Chen et al. 8] do. The reason is that, intuitively, we assume a stronger fairness. Common for the synchronous setting of Dill [12, Alfaro and Henzinger [10, and Chen et al. 8 is that they all have to apply some kind of output pruning: In the composition of two open systems, if a sequence of output transitions leads to an error state, these transitions and the states involved have to be removed.

Compared to our previous work on deadlock freedom in [32], finer trace sets are required to characterize the preorders based on responsiveness. While traces are adequate for the precongruence dealing with deadlock freedom 32 , they do not suffice to characterize the coarsest precongruence for responsiveness, and we had to use some kind of failures instead. In unpublished work, we showed undecidability for the unbounded preorders and precongruences.

The results in Sect. 5 on boundedness without responsiveness are mainly extracted from the bounded variant of deadlock freedom in 32. Brand and Zafiropolu investigated channel boundedness in [4. Haddad et al. [15] study channel properties for asynchronously communicating Petri nets, present compositionality results, and show decidability. The considered properties are concerned with the consumption of messages sent on a channel, such as upper and lower bounds for pending messages, in the presence and absence of fairness. In contrast, boundedness in our setting only restricts the number of pending messages and is, thus, a more abstract and fundamental concept. We do not require that pending messages have to be consumed. Moreover, we assume a strong no- 
tion of fairness by considering a state as satisfactory where it is possible to reach a final state or a state where communication is possible.

The notion of uncoverable states has been observed as the set of certain conflicts by Malik et al. 21] for a stronger correctness criterion than responsiveness. A similar phenomenon occurs in the safe-must preorder [3, where a process and its observer must reach a success state before reaching a catastrophic (i.e., diverging) one.

\section{Conclusion}

We studied an accordance preorder describing whether an open system can safely be replaced by another open system, thereby guaranteeing responsiveness of the overall system. The latter guarantees deadlock freedom and the permanent possibility to mutually communicate. Responsiveness can be seen as a minimal correctness criterion for open systems. We investigated two dimensions of responsiveness, where we can additionally consider final states or require boundedness of the composition due to maintaining a previously known message bound. Altogether, this resulted in four variants of the accordance preorder.

For each variant of accordance, we presented a trace-based characterization. In its basic form, the semantics consist of a set collecting completed traces. In the presence of final states, an additional set is needed to distinguish successfully and unsuccessfully completed traces. To deal with boundedness, we had to add the language and a set of uncoverable traces collecting catastrophic traces that cannot be used reliably.

We showed that none of the four accordance preorders is a precongruence and characterized the coarsest precongruence that is contained in the respective preorder. For basic responsiveness, this precongruence is the should testing preorder 29]. In the presence of final states, it is the should testing preorder extended with traces that do not lead to a final marking. For boundedness, we had to extend the should testing preorder by information about bound violations. In the unbounded setting, we showed in unpublished work that neither the preorders nor the precongruences are decidable. This gives an important motivation to deal with the bounded setting. For the latter setting, we proved decidability of the precongruences.

It is future work to study the relation of our semantics and the compact representation of all controllers in 20. In particular, we are interested in using such representations to decide the coarsest precongruences. Another issue is the minimal requirement weak termination (e.g., [2123]): Reaching a final state should always be possible. This criterion is very close to the idea of should testing, but it is not clear how to characterize the respective accordance (which is

a precongruence itself). In contrast, we characterized precongruences related to responsiveness with semantical ideas that also worked for should testing. 


\section{References}

1. Acciai, L., Boreale, M.: Responsiveness in process calculi. Theor. Comp. Sci. 409(1), 59-93 (2008)

2. Akyildiz, I., Su, W., Sankarasubramaniam, Y., Cayirci, E.: Wireless sensor networks: a survey. Computer Networks 38(4), 393 - 422 (2002)

3. Boreale, M., De Nicola, R., Pugliese, R.: Basic observables for processes. Inf. Comput. 149(1), 77-98 (1999)

4. Brand, D., Zafiropulo, P.: On communicating finite-state machines. J. ACM 30(2), 323-342 (1983)

5. Bravetti, M., Zavattaro, G.: Contract based multi-party service composition. In: FSEN 2007. LNCS, vol. 4767, pp. 207-222 (2007)

6. Brinksma, E., Rensink, A., Vogler, W.: Fair testing. In: CONCUR 1995. LNCS, vol. 962, pp. 313-327. Springer (1995)

7. Brookes, S.D., Hoare, C.A.R., Roscoe, A.W.: A theory of communicating sequential processes. J. ACM 31(3), 560-599 (1984)

8. Chen, T., Chilton, C., Jonsson, B., Kwiatkowska, M.Z.: A compositional specification theory for component behaviours. In: ESOP. LNCS, vol. 7211, pp. 148-168. Springer (2012)

9. Chilton, C., Jonsson, B., Kwiatkowska, M.: An algebraic theory of interface automata. Tech. Rep. RR-13-02, DCS (2013)

10. de Alfaro, L., Henzinger, T.A.: Interface automata. In: ESEC / SIGSOFT FSE 2001. pp. 109-120 (2001)

11. Desai, A., Gupta, V., Jackson, E., Qadeer, S., Rajamani, S.K., Zufferey, D.: P: safe asynchronous event-driven programming. In: PLDI 2013. pp. 321-332. ACM (2013)

12. Dill, D.L.: Trace theory for automatic hierarchical verification of speed-independent circuits. ACM distinguished dissertations, MIT Press (1989)

13. Gamboni, M., Ravara, A.: Responsive choice in mobile processes. In: TGC, LNCS, vol. 6084, pp. 135-152. Springer (2010)

14. Glabbeek, R.J.v.: The coarsest precongruences respecting safety and liveness properties. In: TCS 2010. IFIP, vol. 323, pp. 32-52. Springer (2010)

15. Haddad, S., Hennicker, R., Møller, M.H.: Channel properties of asynchronously composed petri nets. In: Petri Nets. LNCS, vol. 7927, pp. 369-388. Springer (2013)

16. Kobayashi, N.: A type system for lock-free processes. Information and Computation $177(2), 122-159$ (2002)

17. Larsen, K.G.: Modal specifications. In: AVMFSS 1989. LNCS, vol. 407, pp. 232246. Springer (1990)

18. Lohmann, N., Massuthe, P., Wolf, K.: Operating guidelines for finite-state services. In: ICATPN 2007. LNCS, vol. 4546, pp. 321-341. Springer (2007)

19. Lohmann, N., Verbeek, E., Dijkman, R.M.: Petri net transformations for business processes - a survey. In: ToPNoC II. pp. 46-63. LNCS 5460, Springer (2009)

20. Lohmann, N., Wolf, K.: Compact representations and efficient algorithms for operating guidelines. Fundam. Inform. 107, 1-19 (2011)

21. Malik, R., Streader, D., Reeves, S.: Conflicts and fair testing. Journal of Foundations of Computer Science 17(4), 797-813 (2006)

22. Milner, R.: Communication and Concurrency. Prentice-Hall, Inc. (1989)

23. Mooij, A.J., Stahl, C., Voorhoeve, M.: Relating fair testing and accordance for service replaceability. J. Log. Algebr. Program. 79(3-5), 233-244 (2010) 
24. Müller, R.: On the notion of deadlocks in open nets. In: Schwarick, M., Heiner, M. (eds.) AWPN 2010. CEUR Workshop Proceedings, vol. 643, pp. 130-135. CEURWS.org (2010)

25. Natarajan, V., Cleaveland, R.: Divergence and fair testing. In: ICALP. LNCS, vol. 944, pp. 648-659. Springer (1995)

26. Padovani, L.: From lock freedom to progress using session types. To appear for PLACES 2013

27. Papazoglou, M.P.: Web Services: Principles and Technology. Pearson (2007)

28. Reed, J.N., Roscoe, A.W., Sinclair, J.E.: Responsiveness and stable revivals. Formal Asp. Comput. 19(3), 303-319 (2007)

29. Rensink, A., Vogler, W.: Fair testing. Inf. Comput. 205(2), 125-198 (2007)

30. Stahl, C., Massuthe, P., Bretschneider, J.: Deciding substitutability of services with operating guidelines. In: ToPNoC II. pp. 172-191. LNCS 5460, Springer (2009)

31. Stahl, C., Vogler, W.: A trace-based view on operating guidelines. In: FOSSACS 2011. LNCS, vol. 6604, pp. 411-425. Springer (2011)

32. Stahl, C., Vogler, W.: A trace-based service semantics guaranteeing deadlock freedom. Acta Inf. 49(2), 69-103 (2012)

33. Vogler, W.: Modular Construction and Partial Order Semantics of Petri Nets, LNCS, vol. 625. Springer (1992)

34. Vogler, W., Stahl, C., Müller, R.: Trace- and failure-based semantics for bounded responsiveness. Accepted for FOCLASA 2013

35. Vogler, W., Stahl, C., Müller, R.: A trace-based semantics for responsiveness. In: ACSD 2012. pp. 42-51. IEEE (2012)

36. Voorhoeve, M., Mauw, S.: Impossible futures and determinism. Inf. Process. Lett. 80(1), 51-58 (2001)

37. Wolf, K.: Does my service have partners? In: ToPNoC II. pp. 152-171. LNCS 5460, Springer (2009) 